DOI 10.4171/JEMS/256

François Hamel · Lionel Roques

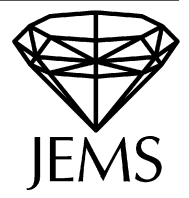

\title{
Uniqueness and stability properties of monostable pulsating fronts
}

Received May 31, 2008 and in revised form June 6, 2009

\begin{abstract}
We prove the uniqueness, up to shifts, of pulsating traveling fronts for reaction-diffusion equations in periodic media with Kolmogorov-Petrovskiī-Piskunov type nonlinearities. These results provide in particular a complete classification of all KPP pulsating fronts. Furthermore, in the more general case of monostable nonlinearities, we also derive several global stability properties and convergence to pulsating fronts for solutions of the Cauchy problem with front-like initial data. In particular, we prove the stability of KPP pulsating fronts with minimal speed, which is a new result even in the case when the medium is invariant in the direction of propagation.
\end{abstract}

\section{Introduction and main results}

This paper is the follow-up of the article [20] on qualitative properties of pulsating traveling fronts in periodic media with monostable reaction terms. By monostable we mean that the fronts connect one unstable limiting state to a weakly stable one. In [20] we proved monotonicity properties and exponential decay of these fronts. Here, we first show the uniqueness of KPP pulsating fronts, for any given speed. The second part of the paper is devoted to further stability properties for solutions of the Cauchy problem with front-like initial data, for general monostable nonlinearities. All these issues have been left open so far and the present paper fills in the main remaining gap in the theory of monostable and specific KPP traveling fronts in periodic media, in the sense that it provides a positive answer to the question of the classification and stability of all KPP pulsating fronts, as well as the stability of pulsating fronts with noncritical speeds in the general monostable framework. Lastly, we point out that, due to our general assumptions on the limiting stationary states, our stability results are new even in the special case of media which are invariant under translation in the direction of propagation. The stability of KPP fronts with minimal speeds involves completely new ideas and is an original result even in the most simplified situations which were previously considered in the literature.

F. Hamel: Aix-Marseille Université and Institut Universitaire de France, LATP, Faculté des Sciences et Techniques, Avenue Escadrille Normandie-Niemen, F-13397 Marseille Cedex 20, France; e-mail: francois.hamel@univ-cezanne.fr

L. Roques: INRA, UR546 Biostatistique et Processus Spatiaux, F-84914 Avignon, France; e-mail: lionel.roques@ avignon.inra.fr 


\subsection{General framework and assumptions}

We consider reaction-diffusion-advection equations of the type

$$
\left\{\begin{array}{l}
u_{t}-\nabla \cdot(A(x, y) \nabla u)+q(x, y) \cdot \nabla u=f(x, y, u), \quad(x, y) \in \bar{\Omega} \\
\nu A(x, y) \nabla u=0, \quad(x, y) \in \partial \Omega
\end{array}\right.
$$

in an unbounded domain $\Omega \subset \mathbb{R}^{N}$ which is assumed to be of class $C^{2, \alpha}$ (with $\alpha>0$ ), periodic in $d$ directions and bounded in the remaining variables. That is, there are an integer $d \in\{1, \ldots, N\}$ and $d$ positive real numbers $L_{1}, \ldots, L_{d}$ such that

$$
\left\{\begin{array}{l}
\exists R \geq 0, \forall(x, y) \in \Omega, \quad|y| \leq R, \\
\forall k \in L_{1} \mathbb{Z} \times \cdots \times L_{d} \mathbb{Z} \times\{0\}^{N-d}, \quad \Omega=\Omega+k
\end{array}\right.
$$

where

$$
x=\left(x_{1}, \ldots, x_{d}\right), \quad y=\left(x_{d+1}, \ldots, x_{N}\right), \quad z=(x, y)
$$

and $|\cdot|$ denotes the euclidean norm. Admissible domains are the whole space $\mathbb{R}^{N}$, the whole space with periodic perforations, infinite cylinders with constant or periodically undulating sections, etc. We denote by $v$ the outward unit normal on $\partial \Omega$, and

$$
\xi B \xi^{\prime}=\sum_{1 \leq i, j \leq N} \xi_{i} B_{i j} \xi_{j}^{\prime}
$$

for any two vectors $\xi=\left(\xi_{i}\right)_{1 \leq i \leq N}$ and $\xi^{\prime}=\left(\xi_{i}^{\prime}\right)_{1 \leq i \leq N}$ in $\mathbb{R}^{N}$ and any $N \times N$ matrix $B=\left(B_{i j}\right)_{1 \leq i, j \leq N}$ with real entries. Throughout the paper, we call

$$
C=\left\{(x, y) \in \bar{\Omega}: x \in\left[0, L_{1}\right] \times \cdots \times\left[0, L_{d}\right]\right\}
$$

the cell of periodicity of $\bar{\Omega}$.

Equations of the type (1.1) arise especially in combustion, population dynamics and ecological models (see e.g. [3, 16, 29, 35, 43, 49]), where $u$ typically stands for the temperature or the concentration of a species.

The symmetric matrix field $A(x, y)=\left(A_{i j}(x, y)\right)_{1 \leq i, j \leq N}$ is of class $C^{1, \alpha}(\bar{\Omega})$ and uniformly positive definite. The vector field $q(x, y)=\left(q_{i}(x, y)\right)_{1 \leq i \leq N}$ is of class $C^{0, \alpha}(\bar{\Omega})$. The nonlinearity $(x, y, u)(\in \bar{\Omega} \times \mathbb{R}) \mapsto f(x, y, u)$ is continuous, of class $C^{0, \alpha}$ with respect to $(x, y)$ locally uniformly in $u \in \mathbb{R}$, and of class $C^{1}$ with respect to $u$. All functions $A_{i j}, q_{i}$ and $f(\cdot, \cdot, u)$ (for all $u \in \mathbb{R}$ ) are assumed to be periodic, in the sense that they satisfy

$$
w(x+k, y)=w(x, y) \quad \text { for all }(x, y) \in \bar{\Omega} \text { and } k \in L_{1} \mathbb{Z} \times \cdots \times L_{d} \mathbb{Z} .
$$

We are given two $C^{2, \alpha}(\bar{\Omega})$ periodic solutions $p^{ \pm}$of the stationary equation

$$
\left\{\begin{array}{l}
-\nabla \cdot\left(A(x, y) \nabla p^{ \pm}\right)+q(x, y) \cdot \nabla p^{ \pm}=f\left(x, y, p^{ \pm}\right) \quad \text { in } \bar{\Omega} \\
\nu A(x, y) \nabla p^{ \pm}=0 \quad \text { on } \partial \Omega
\end{array}\right.
$$


which are ordered, in the sense that $p^{-}(x, y)<p^{+}(x, y)$ for all $(x, y) \in \bar{\Omega} \rrbracket$ We assume that there are $\beta>0$ and $\gamma>0$ such that the function

$$
(x, y, s) \mapsto \frac{\partial f}{\partial u}\left(x, y, p^{-}(x, y)+s\right)
$$

is of class $C^{0, \beta}(\bar{\Omega} \times[0, \gamma])$. Denote

$$
\zeta^{-}(x, y)=\frac{\partial f}{\partial u}\left(x, y, p^{-}(x, y)\right) .
$$

Throughout the paper, we assume that $p^{-}$is linearly unstable in the sense that

$$
\mu^{-}<0
$$

where $\mu^{-}$denotes the principal eigenvalue of the linearized operator around $p^{-}$

$$
\psi \mapsto-\nabla \cdot(A(x, y) \nabla \psi)+q(x, y) \cdot \nabla \psi-\zeta^{-}(x, y) \psi
$$

with periodicity conditions in $\bar{\Omega}$ and the Neumann boundary condition $\nu A \nabla \psi=0$ on $\partial \Omega$. That is, there exists a positive periodic function $\varphi$ in $\bar{\Omega}$ such that

$$
-\nabla \cdot(A(x, y) \nabla \varphi)+q(x, y) \cdot \nabla \varphi-\zeta^{-}(x, y) \varphi=\mu^{-} \varphi \quad \text { in } \bar{\Omega}
$$

and $\nu A(x, y) \nabla \varphi=0$ on $\partial \Omega$. Notice that the condition $\mu^{-}<0$ is fulfilled in particular if $\zeta^{-}(x, y)>0$ for all $(x, y) \in \bar{\Omega}$ or even if $\zeta^{-}$is nonnegative and not identically equal to 0 in $\bar{\Omega}$. We also assume that there is $\rho$ such that

$$
0<\rho<\min _{\bar{\Omega}}\left(p^{+}-p^{-}\right)
$$

and that, for any classical bounded super-solution $\bar{u}$ of

$$
\left\{\begin{array}{l}
\bar{u}_{t}-\nabla \cdot(A(x, y) \nabla \bar{u})+q(x, y) \cdot \nabla \bar{u} \geq f(x, y, \bar{u}) \quad \text { in } \mathbb{R} \times \bar{\Omega}, \\
\nu A \nabla \bar{u} \geq 0 \text { on } \mathbb{R} \times \partial \Omega,
\end{array}\right.
$$

satisfying $\bar{u}<p^{+}$and $\Omega_{\bar{u}}=\left\{(t, x, y) \in \mathbb{R} \times \bar{\Omega}: \bar{u}(t, x, y)>p^{+}(x, y)-\rho\right\} \neq \emptyset$, there exists a family $\left(\rho_{\tau}\right)_{\tau \in[0,1]}$ of functions defined in $\overline{\Omega_{\bar{u}}}$ and satisfying

$$
\left\{\begin{array}{l}
\tau \mapsto \rho_{\tau} \text { is continuous in } C_{t ;(x, y)}^{1+\alpha / 2 ; 2+\alpha}\left(\overline{\Omega_{\bar{u}}}\right), \\
\tau \mapsto \rho_{\tau}(t, x, y) \text { is nondecreasing for each }(t, x, y) \in \overline{\Omega_{\bar{u}}}, \\
\rho_{0}=0, \quad \rho_{1} \geq \rho, \quad \inf _{\Omega_{\bar{u}}} \rho_{\tau}>0 \quad \text { for each } \tau \in(0,1], \\
\left(\bar{u}+\rho_{\tau}\right)_{t}-\nabla \cdot\left(A \nabla\left(\bar{u}+\rho_{\tau}\right)\right)+q \cdot \nabla\left(\bar{u}+\rho_{\tau}\right) \geq f\left(x, y, \bar{u}+\rho_{\tau}\right) \quad \text { in } \Omega_{\bar{u}, \tau}, \\
\nu A \nabla\left(\bar{u}+\rho_{\tau}\right) \geq 0 \quad \text { on }(\mathbb{R} \times \partial \Omega) \cap \Omega_{\bar{u}, \tau},
\end{array}\right.
$$

1 The present paper is concerned with uniqueness and stability properties of pulsating fronts connecting $p^{-}$and $p^{+}$. Under the assumptions below, the fact that these two limiting stationary states are ordered makes the fronts monotone in time, which plays an important role in the proofs. 
where

$$
\Omega_{\bar{u}, \tau}=\left\{(t, x, y) \in \Omega_{\bar{u}}: \bar{u}(t, x, y)+\rho_{\tau}(t, x, y)<p^{+}(x, y)\right\} .
$$

This is a weak stability condition for $p^{+}$. It is satisfied in particular if $p^{+}$is linearly stable (as in Theorem 1.3 below), or if $f$ is nonincreasing in a left neighborhood of $p^{+}$, namely if there exists $\rho \in\left(0, \min _{\bar{\Omega}}\left(p^{+}-p^{-}\right)\right)$such that $f\left(x, y, p^{+}(x, y)+\cdot\right)$ is nonincreasing in $[-\rho, 0]$ for all $(x, y) \in \bar{\Omega}$. It is straightforward to check that condition 1.5$]$ is fulfilled as well if, for every $(x, y) \in \bar{\Omega}$, the function

$$
s \mapsto \frac{f\left(x, y, p^{-}(x, y)+s\right)-f\left(x, y, p^{-}(x, y)\right)}{s}
$$

is nonincreasing in $\left(0, p^{+}(x, y)-p^{-}(x, y)\right)$. Indeed, in this case, we can take any $\rho$ in $\left(0, \min _{\Omega}\left(p^{+}-p^{-}\right)\right)$(see Section 1.1 of [20] for details).

For some of our results, we shall assume a Kolmogorov-Petrovskiǔ-Piskunov type condition on $f$, that is, for all $(x, y) \in \bar{\Omega}$ and $s \in\left[0, p^{+}(x, y)-p^{-}(x, y)\right]$,

$$
f\left(x, y, p^{-}(x, y)+s\right) \leq f\left(x, y, p^{-}(x, y)\right)+\zeta^{-}(x, y) s .
$$

As an example, when $f$ depends on $u$ only and admits two zeroes $p^{-}<p^{+} \in \mathbb{R}$, the above conditions are satisfied if $f$ is of class $C^{1, \beta}$ in a right neighborhood of $p^{-}$with $f^{\prime}\left(p^{-}\right)>0$ and if $f$ is nonincreasing in a left neighborhood of $p^{+}$. The KPP assumption (1.6) reads in this case:

$$
f(u) \leq f^{\prime}\left(p^{-}\right)\left(u-p^{-}\right) \quad \text { for all } u \in\left[p^{-}, p^{+}\right] .
$$

The nonlinearities $f(u)=u(1-u)$ or $f(u)=u(1-u)^{m}$ with $m \geq 1$ are archetype examples (with $p^{-}=0$ and $p^{+}=1$ ) arising in biological models (see [16, 29]).

\subsection{Uniqueness of KPP pulsating fronts}

This paper is concerned with qualitative properties of an important class of solutions of 11.1 , namely the pulsating traveling fronts connecting the two stationary states $p^{-}$ and $p^{+}$. Given a unit vector $e \in \mathbb{R}^{d} \times\{0\}^{N-d}$, a pulsating front connecting $p^{-}$and $p^{+}$, traveling in the direction $e$ with (mean) speed $c \in \mathbb{R}^{*}$, is a time-global classical solution $U(t, x, y)$ of 1.1 such that

$$
\left\{\begin{array}{l}
U(t, x, y)=\phi(x \cdot e-c t, x, y) \text { for all }(t, x, y) \in \mathbb{R} \times \bar{\Omega}, \\
(x, y) \mapsto \phi(s, x, y) \text { is periodic in } \bar{\Omega} \text { for all } s \in \mathbb{R} \\
\phi(s, x, y) \stackrel{s \rightarrow \pm \infty}{\longrightarrow} p^{\mp}(x, y) \quad \text { uniformly in }(x, y) \in \bar{\Omega} \\
p^{-}(x, y)<U(t, x, y)<p^{+}(x, y) \text { for all }(t, x, y) \in \mathbb{R} \times \bar{\Omega} .
\end{array}\right.
$$

With a slight abuse of notation, $x \cdot e$ denotes $x_{1} e_{1}+\cdots+x_{d} e_{d}$, where $e_{1}, \ldots, e_{d}$ are the first $d$ components of the vector $e$. The notion of pulsating traveling fronts extends that of usual traveling fronts which are invariant in the frame moving with speed $c$ in the 
direction $e$. It was proved in [20] that any pulsating front is increasing in time if $c>0$ (or decreasing if $c<0)$. More precisely, $\phi_{s}(s, x, y)<0$ for all $(s, x, y) \in \mathbb{R} \times\left.\bar{\Omega}\right|^{2}$

Our first result yields uniqueness, up to shifts in time, of the pulsating KPP traveling fronts for a given speed $c$ in a given direction $e$.

Theorem 1.1. Let e be a unit vector in $\mathbb{R}^{d} \times\{0\}^{N-d}$, let $c \in \mathbb{R}^{*}$ be given, and assume that the KPP assumption (1.6) is fulfilled. If $U_{1}(t, x, y)=\phi_{1}(x \cdot e-c t, x, y)$ and $U_{2}(t, x, y)=$ $\phi_{2}(x \cdot e-c t, x, y)$ are two pulsating traveling fronts in the sense of $[1.7]$, then there exists $\sigma \in \mathbb{R}$ such that

$$
\phi_{1}(s, x, y)=\phi_{2}(s+\sigma, x, y) \quad \text { for all }(s, x, y) \in \mathbb{R} \times \bar{\Omega},
$$

that is, there exists $\tau \in \mathbb{R}(\tau=-\sigma / c)$ such that

$$
U_{1}(t, x, y)=U_{2}(t+\tau, x, y) \quad \text { for all }(t, x, y) \in \mathbb{R} \times \bar{\Omega} .
$$

As a consequence, in the KPP case, given any direction $e$ and any speed $c \in \mathbb{R}^{*}$, the set of pulsating fronts $U(t, x, y)=\phi(x \cdot e-c t, x, y)$ is either empty or homeomorphic to $\mathbb{R}$. Notice indeed that if $\tau$ is not zero in (1.9), then $U_{1} \neq U_{2}$, since all fronts are strictly monotone in time (see [20]).

The existence of pulsating traveling fronts is known in some cases which are covered by the assumptions of Theorem 1.1. For instance, if

$$
\left\{\begin{array}{l}
p^{-}=0, \quad p^{+}=1, \quad f(x, y, u)>0 \text { for all }(x, y, u) \in \bar{\Omega} \times(0,1), \\
f(x, y, u) \text { is nonincreasing with respect to } u \text { in a left neighborhood of } 1, \\
\nabla \cdot q=0 \text { in } \bar{\Omega}, \quad q \cdot v=0 \text { on } \partial \Omega, \quad \int_{C} q_{i}(x, y) d x d y=0 \text { for } 1 \leq i \leq d,
\end{array}\right.
$$

and if the KPP assumption $(1.6)$ is satisfied, then, given any unit vector $e \in \mathbb{R}^{d} \times\{0\}^{N-d}$, there exists a minimal speed $c^{*}(e)>0$ such that pulsating traveling fronts exist if and only if

$$
c \geq c^{*}(e)=\min _{\lambda>0}(-k(\lambda) / \lambda)=\min \{c \in \mathbb{R}: \exists \lambda>0, k(\lambda)+\lambda c=0\},
$$

where $k(\lambda)$ is the principal eigenvalue of the operator

$$
L_{\lambda} \psi:=-\nabla \cdot(A \nabla \psi)+2 \lambda e A \nabla \psi+q \cdot \nabla \psi+\left[\lambda \nabla \cdot(A e)-\lambda q \cdot e-\lambda^{2} e A e-\zeta^{-}\right] \psi
$$

acting on the set of $C^{2}(\bar{\Omega})$ periodic functions $\psi$ such that $\nu A \nabla \psi=\lambda(\nu A e) \psi$ on $\partial \Omega$ (see [5]; actually, this existence result has been proved under additional smoothness assumptions on the coefficients of 11.1$)$ ). Here $\zeta^{-}(x, y)=\frac{\partial f}{\partial u}(x, y, 0)$. As already emphasized (see Section 1.1 in [20]), conditions (1.6) and (1.10) imply (1.4) and (1.5). Applications of the formula for the minimal speed $c^{*}(e)$ were given in [4, 13, 14, 24, 39, 41, 46, 50].

\footnotetext{
2 In [20], the notation $U(t, x, y)=\phi(c t-x \cdot e, x, y)$ was used, with $\phi( \pm \infty, x, y)=p^{ \pm}(x, y)$. In [20], $\phi$ was then increasing in $s$. The definition (1.7) makes $U$ and $\phi$ face the same direction and is then more natural. In the present paper, the results of [20] have been translated in order to fit with the definition 1.7 .
} 
However, the uniqueness up to shifts for a given speed $c$ was not known. Theorem 1.1 of the present paper thus provides a complete classification of all pulsating fronts: namely, given a direction $e$ in $\mathbb{R}^{d} \times\{0\}^{N-d}$, the set of pulsating fronts is a two-dimensional family, which can be parameterized by the speed $c$ and the shift in the time variable.

For nonlinearities $f$ satisfying $(1.6)$ and 1.10 , the derivative $\zeta^{-}(x, y)=\frac{\partial f}{\partial u}(x, y, 0)$ is positive everywhere. That is why condition (1.4) is fulfilled automatically. However, if $\zeta^{-}$is not everywhere positive, the principal eigenvalue $\mu^{-}$may not be negative in general. In [7], nonlinearities $f=f(x, s)$ (for $x \in \Omega=\mathbb{R}^{N}$ ) satisfying

$$
\left\{\begin{array}{l}
p^{-}=0, \quad f(x, 0)=0, \quad u \mapsto f(x, u) / u \text { is decreasing in } u>0, \\
\exists M>0, \forall x \in \mathbb{R}^{N}, \forall u \geq M, \quad f(x, u) \leq 0
\end{array}\right.
$$

were considered, with no advection $(q=0)$. Typical examples are

$$
f(x, u)=u\left(\zeta^{-}(x)-\eta(x) u\right),
$$

where $\eta$ is a periodic function which is bounded from above and below by two positive constants (see [43] for biological invasion models). Under the assumptions (1.13), the existence (and uniqueness) of a positive periodic steady state $p^{+}$of $(1.2)$ is equivalent to the condition $\mu^{-}<0$, that is, $[1.4$ (see [6]). Notice also that 1.13 implies $[1.5$ (see [20]), as well as $(1.6)$. Under the condition $\mu^{-}<0$, the existence of pulsating fronts in any direction $e$ was proved in [7] for all speeds $c \geq c^{*}(e)$, where $c^{*}(e)$ is still given by (1.11) (see also [25] for partial results in the one-dimensional case), and it was already known from [7] that no pulsating front exists with speed less than $c^{*}(e)$. However, the uniqueness of the front profiles in a given direction $e$ and for a given speed $c \geq c^{*}(e)$ was still an open problem, even in dimension 1.

In short, the first part of the present paper gives a positive answer to the uniqueness issue of the KPP pulsating fronts, in a setting which unifies and is more general than 1.10 or (1.13). In particular, in this paper, the nonlinearity $f$ is not assumed to be nonnegative or to satisfy any monotonicity properties. Actually, Theorem 1.1 follows from a more general uniqueness result which does not require the KPP assumption (1.6) but needs additional a priori properties for any two fronts with the same given speed: see Theorem 2.2 in Section 2 .

Remark 1.2. If both $p^{-}$and $p^{+}$are weakly stable - that is, when 1.5 is satisfied and when the instability assumption 1.4 of $p^{-}$is replaced by a weak stability assumption which is similar to (1.5) - then the analysis is much easier. Comparison principles such as Lemma 2.1 below, which can be viewed as weak maximum principles in some unbounded domains, would then hold not only in the region where the solutions are close to $p^{+}, b u t$ also in the region where they are close to $p^{-}$. Two given fronts could then automatically be compared globally in $\mathbb{R} \times \bar{\Omega}$, up to time-shifts, and a sliding method similar to [2, 3] would imply that the functions $\phi(s, x, y)$ are unique up to shifts in the variable $s$, and that the speed $c$, if any, is necessarily unique. This is the case for instance for bistable or combustion-type nonlinearities (see [2, 3, 9, 32, 34, 47, 48, 49] for existence and further qualitative results with such reaction terms). In the present paper, as a consequence of the 
instability of $p^{-}$, one cannot use versions of the weak maximum principles in the region where the solutions are close to $p^{-}$. Therefore, even if the proofs of Theorems 1.1 and 2.2 below are based on a sliding method, the main difficulty is to compare two given fronts globally and especially to compare their tails in the region where they approach $p^{-}$(see Section 2 for further details).

\subsection{Global stability of KPP or general monostable fronts}

The second part of this paper is concerned with stability issues for KPP or general monostable fronts. The stability of the fronts and the convergence to them at large times is indeed one of the most important features of reaction-diffusion equations. We are back to the general periodic framework and we shall see that, under some assumptions on the initial conditions, solutions of the Cauchy problem (1.1) will converge to pulsating fronts.

To state the stability results, we need a few more notations. In what follows, $e$ denotes a given unit vector in $\mathbb{R}^{d} \times\{0\}^{N-d}$ and $\zeta^{-}(x, y)$ is defined as in 1.3 . For each $\lambda \in \mathbb{R}$, denote by $k(\lambda)$ the principal eigenvalue of the operator $L_{\lambda}$ defined in 1.12 and let $\psi_{\lambda}$ denote the unique positive principal eigenfunction of $L_{\lambda}$ such that, say,

$$
\left\|\psi_{\lambda}\right\|_{L^{\infty}(\Omega)}=1 .
$$

It has been proved (see Proposition 1.2 in [20]) that, for any pulsating traveling front $U(t, x, y)=\phi(x \cdot e-c t, x, y)$ of (1.1) in the sense of (1.7),

$$
c \geq c^{*}(e):=\inf _{\lambda>0}(-k(\lambda) / \lambda)
$$

The quantity $c^{*}(e)$ is a real number, and for each $c>c^{*}(e)$, the positive real number

$$
\lambda_{c}=\min \{\lambda>0: k(\lambda)+c \lambda=0\}
$$

is well-defined (see [20]).

Let now $\mu^{+}$be the principal eigenvalue of the linearized operator

$$
\psi \mapsto-\nabla \cdot(A(x, y) \nabla \psi)+q(x, y) \cdot \nabla \psi-\frac{\partial f}{\partial u}\left(x, y, p^{+}(x, y)\right) \psi
$$

around the limiting state $p^{+}$, with periodicity conditions in $\bar{\Omega}$ and the Neumann boundary condition $v A \nabla \psi=0$ on $\partial \Omega$. Let $\psi^{+}$be the unique positive principal eigenfunction such that $\left\|\psi^{+}\right\|_{L^{\infty}(\Omega)}=1$. The function $\psi^{+}$satisfies

$$
\left\{\begin{array}{l}
-\nabla \cdot\left(A(x, y) \nabla \psi^{+}\right)+q(x, y) \cdot \nabla \psi^{+}-\frac{\partial f}{\partial u}\left(x, y, p^{+}(x, y)\right) \psi^{+}=\mu^{+} \psi^{+} \text {in } \bar{\Omega}, \\
\psi^{+}>0 \text { in } \bar{\Omega}, \quad \max _{\bar{\Omega}} \psi^{+}=1, \\
\nu A \nabla \psi^{+}=0 \quad \text { on } \partial \Omega .
\end{array}\right.
$$

It is straightforward to check (see [20]) that the condition $\mu^{+}>0$ implies the weak stability property 1.5 . 
From now on, $u_{0}$ denotes a uniformly continuous function defined in $\bar{\Omega}$ such that

$$
p^{-}(x, y) \leq u_{0}(x, y) \leq p^{+}(x, y) \quad \text { for all }(x, y) \in \bar{\Omega},
$$

and let $u(t, x, y)$ be the solution of the Cauchy problem 1.1 for $t>0$, with initial condition $u_{0}$ at time $t=0$. Observe that

$$
p^{-}(x, y) \leq u(t, x, y) \leq p^{+}(x, y)
$$

for all $(x, y) \in \bar{\Omega}$ and $t \geq 0$, from the maximum principle.

The following theorem is concerned with the global stability of general monostable pulsating fronts for speeds larger than $c^{*}(e)$.

Theorem 1.3. Assume that $\mu^{+}>0$ and that $U(t, x, y)=\phi(x \cdot e-c t, x, y)$ is a pulsating traveling front with speed $c>c^{*}(e)$ such that

$$
\lim _{s \rightarrow+\infty} \sup _{(x, y) \in \bar{\Omega}}\left|\frac{\ln \left(\phi(s, x, y)-p^{-}(x, y)\right)}{s}+\lambda_{c}\right|=0 .
$$

Then there exists $\varepsilon_{0}>0$ such that if

$$
\liminf _{\varsigma \rightarrow-\infty} \inf _{(x, y) \in \bar{\Omega}, x \cdot e \leq \varsigma}\left[u_{0}(x, y)-p^{+}(x, y)\right]>-\varepsilon_{0}
$$

and

$$
u_{0}(x, y)-p^{-}(x, y) \sim U(0, x, y)-p^{-}(x, y) \quad \text { as } x \cdot e \rightarrow+\infty, 3
$$

then

$$
\sup _{(x, y) \in \bar{\Omega}}|u(t, x, y)-U(t, x, y)| \rightarrow 0 \quad \text { as } t \rightarrow+\infty .
$$

In Theorem 1.3, the assumption 1.18 on the logarithmic equivalent of $\phi(s, x, y)-$ $p^{-}(x, y)$ as $s \rightarrow+\infty$ is automatically satisfied under the KPP condition 1.6 (see formulas $(1.22)$ and $(1.23)$ below and Theorem 1.5$)$. Actually, assumption 1.6 is not required here and it is only assumed that the limiting state $p^{-}$is unstable while the other one, $p^{+}$, is stable. But it does not mean a priori that $f$ is of the KPP type or that there is no other stationary state $p$ between $p^{-}$and $p^{+}$. In the general monostable case, assumption (1.18) is also fulfilled, without the KPP condition, as soon as there exists a pulsating front

$$
U^{\prime}(t, x, y)=\phi^{\prime}\left(x \cdot e-c^{\prime} t, x, y\right)
$$

in the sense of $\left[1.7\right.$ with a speed $c^{\prime}<c$ (see Theorem 1.5 in [20]). As a consequence, the following corollary holds.

\footnotetext{
3 Condition 1.20 is understood as $\sup _{(x, y) \in \bar{\Omega}, x \cdot e \geq 5} \mid\left(u_{0}(x, y)-p^{-}(x, y)\right) /(U(0, x, y)-$ $\left.p^{-}(x, y)\right)-1 \mid \rightarrow 0$ as $\varsigma \rightarrow+\infty$.
} 
Corollary 1.4. In Theorem 1.3 if the assumption (1.18) is replaced by the existence of a pulsating front $U^{\prime}(t, x, y)=\phi^{\prime}\left(x \cdot e-c^{\prime} t, x, y\right)$ with a speed $c^{\prime}<c$, then the conclusion still holds.

The existence of a pulsating front with a speed $c^{\prime}<c$ is a reasonable assumption. For instance, under assumptions 1.10 with $\frac{\partial f}{\partial u}(x, y, 0)>0$, even without the KPP assumption (1.6), pulsating fronts $U(t, x, y)=\phi(x \cdot e-c t, x, y)$ exist if and only if $c \geq c^{* *}(e)$, where the minimal speed $c^{* *}(e)$ is such that $c^{* *}(e) \geq c^{*}(e)$ and $c^{*}(e)$ is given in 1.11 (see [2, 3] ). Thus, for each $c>c^{* *}(e)$, the existence of a pulsating traveling front with a speed $c^{\prime}<c$ is guaranteed.

Let us now comment on Theorem 1.3 and Corollary 1.4 and give some insight into their proofs. These two statements are global stability results for general monostable fronts. The initial condition $u_{0}$ is in some sense close to the pulsating front $U(0, \cdot, \cdot)$ at both ends, that is, when $x \cdot e \rightarrow \pm \infty$. Assumption (1.19) means that $u_{0}$ has to be in the basin of attraction of the stable state $p^{+}$as $x \cdot e$ is very negative. But these conditions are not very restrictive and $u_{0}$ is not required to be close to $U(0, \cdot, \cdot)$ when $|x \cdot e|$ is not large. Nevertheless, the convergence result $(1.21)$ as $t \rightarrow+\infty$ is uniform in space. The assumptions of Theorem 1.3 and Corollary 1.4 alone imply that the solution $u(t, x, y)$ converges to the periodicity condition — namely the second property of (1.7p —asymptotically as $t \rightarrow+\infty$, whereas $u_{0}$ does not satisfy any such periodicity condition. A serious difficulty in Cauchy problems of the type (1.1) is indeed to get uniform estimates in the variables which are orthogonal to the direction $e$ (establishing such estimates is an essential tool in the proof of Theorem 1.3 . This difficulty was not present in the case of one-dimensional media or infinite cylinders with bounded sections, because of the compactness of cross sections.

The general strategy of the proofs is, as in the paper by Fife and McLeod [15], to trap the solution $u(t, x, y)$ between suitable sub- and super-solutions which are close to some shifts of the pulsating traveling front $U$, and then to show that the shifts can be chosen as small as we want when $t \rightarrow+\infty$. However, the method is much more involved than in the bistable case investigated in [15]: not only does the instability of $p^{-}$ require more precise estimates in the region where $x \cdot e-c t$ is positive, but also the fact that $p^{+}$is only assumed to be stable (in the sense that $\mu^{+}>0$ ) without any sign hypothesis on $f(\cdot, \cdot, s)-f\left(\cdot, \cdot, p^{+}\right)$as $s \simeq p^{+}$makes the situation more complicated and requires the use of the principal eigenfunction $\psi^{+}$in the definition of the sub- and super-solutions (dealing here with the general monostable case introduces additional difficulties which would not be present in the KPP case, especially as far as super-solutions are concerned). Furthermore, the dependence of all coefficients $A, q$ and $f$ on the spatial variables $(x, y)$ induces additional technical difficulties, which are overcome by the use of space-dependent exponential correcting terms (we refer to Section 3 for further details).

Lastly, it is worth pointing out that there is no shift in the limiting profile, unlike for combustion-type or bistable equations (we refer to [15, 27, 40] for results with such nonlinearities in the one-dimensional case, or in infinite cylinders with invariance under translation in the direction of propagation; see equation (1.28) below). 
Let us now deal with the particular KPP case 1.6. The assumptions of Theorem 1.3 can then be rewritten in a more explicit way. We first recall that, under the assumption (1.6), if $c>c^{*}(e)$ then

$$
\phi(s, x, y)-p^{-}(x, y) \sim B_{\phi} e^{-\lambda_{c} s} \psi_{\lambda_{c}}(x, y) \quad \text { as } s \rightarrow+\infty
$$

uniformly in $(x, y) \in \bar{\Omega}$, for some $B_{\phi}>0$, while if $c=c^{*}(e)$ then there is a unique $\lambda^{*}>0$ such that $k\left(\lambda^{*}\right)+c^{*}(e) \lambda^{*}=0$ and there exists $B_{\phi}>0$ such that

$$
\phi(s, x, y)-p^{-}(x, y) \sim B_{\phi} s^{2 m+1} e^{-\lambda^{*} s} \psi_{\lambda^{*}}(x, y) \quad \text { as } s \rightarrow+\infty
$$

uniformly in $(x, y) \in \bar{\Omega}$, where $m \in \mathbb{N}$ and $2 m+2$ is the multiplicity of $\lambda^{*}$ as a root of $k(\lambda)+c^{*}(e) \lambda=0$ (see Theorem 1.3 in [20]).

Theorem 1.5. Assume that the KPP condition 1.6 is satisfied, that $\mu^{+}>0$ and that $U(t, x, y)=\phi(x \cdot e-c t, x, y)$ is a pulsating traveling front of 1.1 . Then there is $\varepsilon_{0}>0$ such that the following holds.

(1) If $c>c^{*}(e)$, if $u_{0}$ satisfies (1.19) and if there exists $B>0$ such that

$$
u_{0}(x, y)-p^{-}(x, y) \sim B e^{-\lambda_{c} x \cdot e} \psi_{\lambda_{c}}(x, y) \quad \text { as } x \cdot e \rightarrow+\infty,
$$

then

$$
\sup _{(x, y) \in \bar{\Omega}}|u(t, x, y)-U(t+\tau, x, y)| \rightarrow 0 \quad \text { as } t \rightarrow+\infty \text {, }
$$

where $\tau$ is the unique real number such that $B_{\phi} e^{\lambda_{c} c \tau}=B$ and $B_{\phi}>0$ is given by (1.22).

(2) If $c=c^{*}(e)$, if $u_{0}$ satisfies (1.19) and if there exists $B>0$ such that

$$
u_{0}(x, y)-p^{-}(x, y) \sim B(x \cdot e)^{2 m+1} e^{-\lambda^{*} x \cdot e} \psi_{\lambda^{*}}(x, y) \quad \text { as } x \cdot e \rightarrow+\infty,
$$

then 1.25 holds, where $\tau$ is the unique real number such that $B_{\phi} e^{\lambda^{*} c^{*}(e) \tau}=B$ and $B_{\phi}>0$ is given by (1.23).

It is immediate to see that, under the notations of Theorem 1.5

$$
u_{0}(x, y)-p^{-}(x, y) \sim U(\tau, x, y)-p^{-}(x, y) \quad \text { as } x \cdot e \rightarrow+\infty .
$$

As a consequence, part (1) of Theorem 1.5 is a corollary of Theorem 1.3. Part (2) is more technical and needs a specific proof, which is done in Section 4 . The main additional difficulty relies on the fact that the exponentially decaying functions $e^{-\lambda^{*} s}$ characterizing the behavior of KPP fronts with minimal speeds near the unstable steady state $p^{-}$are multiplied by polynomial pre-factors. These pre-factors vanish somewhere. The construction of sub- and super-solutions must take this fact into account and it is therefore much more intricate. The sub- and super-solutions used in the proof use extra polynomial times exponentially decaying terms involving some derivatives of the principal eigenfunctions $\psi_{\lambda}$ with respect to $\lambda$ at the critical rate $\lambda=\lambda^{*}$. We point out that these ideas are new even in the previous special cases which were investigated in the literature. 
From Corollary 1.4 and Theorem 1.5 it follows that the only case which is not covered by our stability results is the monostable case without the KPP assumption (1.6) and when the front $U$ is the slowest one among all pulsating fronts. The situation is different in this case, and in general a shift in time is expected to occur in the convergence to the front at large times, like in combustion-type nonlinearities.

It can be seen from Theorems 1.3 and 1.5 that the propagation speed of $u(t, x, y)$ at large times strongly depends on the asymptotic behavior of the initial condition $u_{0}$ when it approaches the unstable state $p^{-}$. Actually, this fact has already been known in some simpler situations. In particular, the above stability results extend earlier ones for the usual traveling fronts $U(t, x)=\phi(x-c t)$ of the homogeneous one-dimensional equation

$$
u_{t}=u_{x x}+f(u) \quad \text { in } \mathbb{R}
$$

with $f(0)=f(1)=0\left(p^{-}=0\right.$ and $\left.p^{+}=1\right)$ and $f>0$ in $(0,1)$, with or without the KPP condition $0<f(s) \leq f^{\prime}(0) s$ in $(0,1)$ (see e.g. [10, 18, 26, 30, 31, 42, 44, 45]). In this case, the minimal speed is equal to $c^{*}=2 \sqrt{f^{\prime}(0)}, k(\lambda)=-\lambda^{2}-f^{\prime}(0)$ for each $\lambda \in \mathbb{R}, \lambda^{*}=\sqrt{f^{\prime}(0)}$ and $m=0$. Theorem 1.5 also generalizes the stability results for the traveling fronts $U(t, x, y)=\phi(x-c t, y)$ (which are still invariant in their moving frame) of equations of the type

$$
u_{t}-\Delta u+\alpha(y) \frac{\partial u}{\partial x}=f(u),(x, y) \in \Omega=\mathbb{R} \times \omega, \quad v \cdot \nabla u=0,(x, y) \in \partial \Omega
$$

in straight infinite cylinders with smooth bounded sections $\omega$ and with underlying shear flows $q=(\alpha(y), 0, \ldots, 0)$, for nonlinearities $f$ such that $f(0)=f(1)=0$ and satisfying the stronger KPP assumption that $f(s) / s$ is nonincreasing in $(0,1)$ (see [33]). For equations (1.28), we refer to [9] for existence and uniqueness results of traveling fronts. Some stability results without the KPP assumption (when 0 and 1 are assumed to be the only possible steady states in [0,1]) have also been established in [33] and [40]. Recently, stability results for the one-dimensional equation

$$
u_{t}=u_{x x}+f(x, u)
$$

with KPP periodic nonlinearity $f(x, u)$ have been obtained in [1] with the use of Floquet exponents. The stability and uniqueness of one-dimensional pulsating KPP fronts for discretized equations have just been addressed in [19], under the assumption of exponential behavior of the fronts when they approach the unstable state. Actually, we point out that, in the KPP case, even for the equation (1.28) in infinite cylinders or for the onedimensional periodic discrete or continuous framework, the question of stability of fronts with minimal speed has been open. Part (2) of Theorem 1.5 gives a positive answer to this important question.

The general philosophy of the aforementioned references [1, 33, 40] is that, if the initial condition $u_{0}$ approaches the unstable state $p^{-}=0$ like a (pulsating) traveling front up to a faster exponential term, then the convergence of $u$ to the front at large times is exponential in time in weighted function spaces. The method is based on spectral properties in weighted spaces and it also uses the exact exponential behavior of the fronts 
when they approach 0 . We conjecture that such a more precise convergence result holds in our general periodic framework-at least in the KPP case when the exact exponential behavior is known-under a stronger assumption on $u_{0}$, like $u_{0}(x, y)-p^{-}(x, y)=$ $U(0, x, y)-p^{-}(x, y)+O\left(\left(U(0, x, y)-p^{-}(x, y)\right)^{1+\varepsilon}\right)$ as $x \cdot e \rightarrow+\infty$, for some $\varepsilon>0$. However, this is not the purpose of the present paper and the method which we use to prove Theorems 1.3 and 1.5 is based directly on the construction of suitable sub- and super-solutions and on some Liouville type results. Furthermore, the method works in the general monostable periodic framework and it only requires that

$$
u_{0}(x, y)-p^{-}(x, y) \sim U(0, x, y)-p^{-}(x, y) \quad \text { as } x \cdot e \rightarrow+\infty,
$$

as well as the logarithmic equivalent of the fronts when they approach the unstable state $p^{-}$. However, in Theorems 1.3 and 1.5 , the assumptions $1.20,, 1.24$ and 1.26 play an essential role and cannot be relaxed. Indeed, with a KPP type nonlinearity $f$, for equation 1.29, if $u_{0}(x)$ is simply assumed to be trapped between two shifts of a front $\phi$, then $u$ may exhibit nontrivial dynamics and its $\omega$-limit set may be a continuum of translates of $\phi$ (see [1]). On the other hand, even in the homogeneous one-dimensional case (1.27), if $u_{0}(x)$ is just assumed to be trapped as $x \cdot e \rightarrow+\infty$ between two exponentially decaying functions with two different decay rates, the asymptotic propagation speed of $u$ as $t \rightarrow+\infty$ may not be unique in general (see [21] for details; see also [23] for results in the same spirit for combustion-type equations). Lastly, if $u_{0}(x)$ decays more slowly than any exponentially decaying function as $x \cdot e \rightarrow+\infty$, then the asymptotic propagation speed is infinite (see [11, 22]).

\subsection{Additional results in the time-periodic case}

Finally, we mention that, with the same type of methods as in this paper, similar uniqueness and stability results can be established for pulsating fronts in time-periodic media (however, in order not to lengthen this paper, we just state the conclusions without the detailed proofs). Namely, consider reaction-diffusion-advection equations of the type

$$
\left\{\begin{array}{l}
u_{t}-\nabla \cdot(A(t, y) \nabla u)+q(t, y) \cdot \nabla u=f(t, y, u) \text { in } \bar{\Omega} \\
v A \nabla u=0 \text { on } \partial \Omega
\end{array}\right.
$$

in a smooth unbounded domain $\Omega=\left\{(x, y) \in \mathbb{R}^{d} \times \omega\right\}$, where $\omega$ is a $C^{2, \alpha}$ bounded domain of $\mathbb{R}^{N-d}$. The uniformly elliptic symmetric matrix field $A(t, y)=\left(A_{i j}(t, y)\right)_{1 \leq i, j \leq N}$ is of class $C_{t ; y}^{1, \alpha / 2 ; 1, \alpha}(\mathbb{R} \times \bar{\omega})$, the vector field $q(t, y)=\left(q_{i}(t, y)\right)_{1 \leq i \leq N}$ is of class $C_{t ; y}^{0, \alpha / 2 ; 1, \alpha}(\mathbb{R} \times \bar{\omega})$ and the nonlinearity $(t, y, u)(\in \mathbb{R} \times \bar{\omega} \times \mathbb{R}) \mapsto f(t, y, u)$ is continuous, of class $C^{0, \alpha / 2 ; 0, \alpha}$ with respect to $(t, y)$ locally uniformly in $u \in \mathbb{R}$ and of class $C^{1}$ with respect to $u$ in $\mathbb{R} \times \bar{\omega} \times \mathbb{R}$. All functions $A_{i j}, q_{i}$ and $f(\cdot, \cdot, u$ ) (for all $u \in \mathbb{R}$ ) are assumed to be time-periodic, in the sense that they satisfy $w(t+T, y)=w(t, y)$ for all $(t, y) \in \mathbb{R} \times \bar{\omega}$, where $T>0$ is given. We are given two time-periodic classical solutions $p^{ \pm}$of 1.30 satisfying

$$
p^{-}(t, y)<p^{+}(t, y) \quad \text { for all }(t, y) \in \mathbb{R} \times \bar{\omega} .
$$


Assume that the function $(t, y, s) \mapsto \frac{\partial f}{\partial u}\left(t, y, p^{-}(t, y)+s\right)$ is of class $C^{0, \beta}(\mathbb{R} \times \bar{\omega} \times[0, \gamma])$ for some $\beta>0$ and $\gamma>0$, and that $\mu^{-}<0$, where $\mu^{-}$denotes the principal eigenvalue of the linearized operator around $p^{-}$,

$$
\psi(t, y) \mapsto \psi_{t}-\nabla \cdot(A(t, y) \nabla \psi)+q(t, y) \cdot \nabla \psi-\frac{\partial f}{\partial u}\left(t, y, p^{-}(t, y)\right) \psi,
$$

with time-periodicity conditions in $\mathbb{R} \times \bar{\omega}$ and Neumann boundary condition $\nu A \nabla \psi=0$ on $\mathbb{R} \times \partial \omega$. With a slight abuse of notation, $\nabla \psi$ denotes $\left(0, \ldots, 0, \nabla_{y} \psi\right) \in\{0\}^{d} \times \mathbb{R}^{N-d}$. Assume that there is $\rho$ such that $0<\rho<\min _{\mathbb{R} \times \bar{\omega}}\left(p^{+}-p^{-}\right)$and, for any classical bounded supersolution $\bar{u}$ of 1.30 satisfying $\bar{u}<p^{+}$and $\Omega_{\bar{u}}=\{\bar{u}(t, x, y)>$ $\left.p^{+}(t, y)-\rho\right\} \neq \varnothing$, there exists a family $\left(\rho_{\tau}\right)_{\tau \in[0,1]}$ of functions defined in $\overline{\Omega_{\bar{u}}}$ and satisfying 1.5 with $\Omega_{\bar{u}, \tau}=\left\{(t, x, y) \in \Omega_{\bar{u}}: \bar{u}(t, x, y)+\rho_{\tau}(t, x, y)<p^{+}(t, y)\right\}$. The KPP condition $(1.6)$ is replaced with the following one: for all $(t, y) \in \mathbb{R} \times \bar{\omega}$ and $s \in\left[0, p^{+}(t, y)-p^{-}(t, y)\right]$,

$$
f\left(t, y, p^{-}(t, y)+s\right) \leq f\left(t, y, p^{-}(t, y)\right)+\frac{\partial f}{\partial u}\left(t, y, p^{-}(t, y)\right) s .
$$

Given a unit vector $e \in \mathbb{R}^{d} \times\{0\}^{N-d}$, a pulsating front connecting $p^{-}$and $p^{+}$, traveling in the direction $e$ with mean speed $c \in \mathbb{R}^{*}$, is a classical solution $U(t, x, y)$ of $(1.30)$ such that

$$
\left\{\begin{array}{l}
U(t, x, y)=\phi(x \cdot e-c t, t, y) \quad \text { for all }(t, x, y) \in \mathbb{R} \times \mathbb{R}^{d} \times \bar{\omega} \\
\phi(s, t+T, y)=\phi(s, t, y) \quad \text { for all }(s, t, y) \in \mathbb{R}^{2} \times \bar{\omega} \\
\phi(s, t, y) \stackrel{s \rightarrow \pm \infty}{\longrightarrow} p^{\mp}(t, y) \quad \text { uniformly in }(t, y) \in \mathbb{R} \times \bar{\omega} \\
p^{-}(t, y)<U(t, x, y)<p^{+}(t, y) \quad \text { for all }(t, x, y) \in \mathbb{R} \times \mathbb{R}^{d} \times \bar{\omega}
\end{array}\right.
$$

We refer to [17, 37, 38] for existence results and speed estimates of pulsating fronts for equations of the type $(1.30)$ with time-periodic KPP nonlinearities and shear flows (see also [36] for the existence of fronts in space-time periodic media). For each $\lambda \in \mathbb{R}$, still define $k(\lambda)$ as the principal eigenvalue of the operator

$$
\begin{aligned}
\psi \mapsto \psi_{t}-\nabla \cdot(A \nabla \psi)+2 \lambda e A \nabla \psi+q \cdot \nabla \psi \\
+\left[\lambda \nabla \cdot(A e)-\lambda q \cdot e-\lambda^{2} e A e-\frac{\partial f}{\partial u}\left(t, y, p^{-}(t, y)\right)\right] \psi
\end{aligned}
$$

with time-periodicity conditions in $\mathbb{R} \times \bar{\omega}$ and the boundary conditions $\nu A \nabla \psi=\lambda(\nu A e) \psi$ on $\mathbb{R} \times \partial \omega$, and denote by $\psi_{\lambda}$ the unique positive principal eigenfunction such that $\left\|\psi_{\lambda}\right\|_{L^{\infty}(\mathbb{R} \times \omega)}=1$. Define $c^{*}(e)$ as in 1.15 and for each $c>c^{*}(e)$, define $\lambda_{c}>0$ as in (1.16). These quantities are well-defined real numbers.

Then, for any pulsating traveling front, one has $c \geq c^{*}(e)$ (this fact was already mentioned in [20]). Furthermore, under the KPP assumption (1.31], if

$$
U_{1}(t, x, y)=\phi_{1}(x \cdot e-c t, t, y) \quad \text { and } \quad U_{2}(t, x, y)=\phi_{2}(x \cdot e-c t, t, y)
$$


are two pulsating traveling fronts with the same speed $c$, then $\phi_{1}(s, t, y)=\phi_{2}(s+\sigma, t, y)$ in $\mathbb{R}^{2} \times \bar{\omega}$ for some $\sigma \in \mathbb{R}$.

In the following, assume that $\mu^{+}>0$, where $\mu^{+}$denotes the principal eigenvalue of the linearized operator around $p^{+}$,

$$
\psi(t, y) \mapsto \psi_{t}-\nabla \cdot(A(t, y) \nabla \psi)+q(t, y) \cdot \nabla \psi-\frac{\partial f}{\partial u}\left(t, y, p^{+}(t, y)\right) \psi,
$$

with time-periodicity in $\mathbb{R} \times \bar{\omega}$ and the Neumann boundary condition $\nu A \nabla \psi=0$ on $\mathbb{R} \times \partial \omega$. Consider a pulsating front $U(t, x, y)=\phi(x \cdot e-c t, t, y)$ in the sense of [1.32).

If $c>c^{*}(e)$ and if $\ln \left(\phi(s, t, y)-p^{-}(t, y)\right) \sim-\lambda_{c} s$ as $s \rightarrow+\infty$ uniformly in $(t, y) \in \mathbb{R} \times \bar{\omega}$, then there exists $\varepsilon_{0}>0$ such that, for any uniformly continuous function $u_{0}$ such that

$$
\left\{\begin{array}{l}
p^{-}(\tau, y) \leq u_{0}(x, y) \leq p^{+}(\tau, y) \quad \text { for all }(x, y) \in \bar{\Omega} \\
\liminf _{\varsigma \rightarrow-\infty} \inf _{(x, y) \in \bar{\Omega}, x \cdot e \leq \varsigma}\left[u_{0}(x, y)-p^{+}(\tau, y)\right]>-\varepsilon_{0}
\end{array}\right.
$$

and $u_{0}(x, y)-p^{-}(\tau, y) \sim U(\tau, x, y)-p^{-}(\tau, y)$ as $x \cdot e \rightarrow+\infty$ for some $\tau \in \mathbb{R}$, the solution $u(t, x, y)$ of 1.30 with initial condition $u_{0}$ satisfies

$$
\sup _{(x, y) \in \bar{\Omega}}|u(t, x, y)-U(t+\tau, x, y)| \rightarrow 0 \quad \text { as } t \rightarrow+\infty .
$$

Lastly, under the KPP condition (1.31), there is $\varepsilon_{0}>0$ such that the following holds. If $c>c^{*}(e)$ and if there exist $\tau \in \mathbb{R}$ and $B>0$ such that $u_{0}$ satisfies (1.33) and $u_{0}(x, y)-p^{-}(\tau, y) \sim B e^{-\lambda_{c} x \cdot e} \psi_{\lambda_{c}}(\tau, y)$ as $x \cdot e \rightarrow+\infty$, then the solution $u(t, x, y)$ of (1.30) with initial condition $u_{0}$ satisfies

$$
\sup _{(x, y) \in \bar{\Omega}}|u(t, x, y)-U(t+\tau, x+\sigma e, y)| \rightarrow 0 \quad \text { as } t \rightarrow+\infty
$$

where $\sigma$ is the unique real number such that $B_{\phi} e^{\lambda_{c}(c \tau-\sigma)}=B$ and $B_{\phi}>0$ is given by $\phi(s, t, y)-p^{-}(t, y) \sim B_{\phi} e^{-\lambda_{c} s} \psi_{\lambda_{c}}(t, y) \quad$ as $s \rightarrow+\infty$ uniformly in $(t, y) \in \mathbb{R} \times \bar{\omega}$.

On the other hand, if $c=c^{*}(e)$ and if there exist $\tau \in \mathbb{R}$ and $B>0$ such that $u_{0}$ satisfies 1.33 and $u_{0}(x, y)-p^{-}(\tau, y) \sim B(x \cdot e)^{2 m+1} e^{-\lambda^{*} x \cdot e} \psi_{\lambda^{*}}(\tau, y)$ as $x \cdot e \rightarrow+\infty$, where $\lambda^{*}$ is the unique positive root of $k(\lambda)+c^{*}(e) \lambda=0$, with multiplicity $2 m+2$, then 1.34 holds, where $\sigma$ satisfies $B_{\phi} e^{\lambda^{*}\left(c^{*}(e) \tau-\sigma\right)}=B$ and $B_{\phi}>0$ is given by

$\phi(s, t, y)-p^{-}(t, y) \sim B_{\phi} s^{2 m+1} e^{-\lambda^{*} s} \psi_{\lambda^{*}}(t, y)$ as $s \rightarrow+\infty$ uniformly in $(t, y) \in \mathbb{R} \times \bar{\omega}$.

Outline of the paper. Section 2 is devoted to the uniqueness results. In Section 3 , the proof of the stability result in the general monostable case is given. Lastly, Section 4 is concerned with the proof of the stability of KPP fronts with minimal speed $c^{*}(e)$. 


\section{Uniqueness of the fronts up to shifts}

This section is devoted to the proof of the uniqueness result, that is, Theorem 1.1 Theorem 1.1 is itself based on another uniqueness result which is valid in the general monostable case. The basic strategy is to compare a given front $\phi_{2}$ with to the shifts of another one $\phi_{1}$ and to prove that, for a critical shift, the two fronts are identically equal. In other words, we use a sliding method. One of the difficulties is to initiate the sliding method, that is, to compare the solutions globally in $\mathbb{R} \times \bar{\Omega}$, and in particular in the region where both fronts are close to $p^{-}$(as $s \rightarrow+\infty$ ). In this region, the weak maximum principle does not hold because of the instability of $p^{-}$. However, this difficulty can be overcome because the fronts have a nondegenerate behavior as $s \rightarrow+\infty$ (see (2.2) below).

Before doing so, we first quote from [20] a useful lemma (see Lemma 2.3 in [20]) which is a comparison result between sub- and super-solutions in the region where $s \leq h$.

Lemma 2.1. Let $\rho \in\left(0, \min _{\bar{\Omega}}\left(p^{+}-p^{-}\right)\right)$be as in $\sqrt{1.5}$. Let $\bar{U}$ and $\underline{U}$ be respectively $a$ classical super-solution and sub-solution of

$$
\left\{\begin{array}{l}
\bar{U}_{t}-\nabla \cdot(A(x, y) \nabla \bar{U})+q(x, y) \cdot \nabla \bar{U} \geq f(x, y, \bar{U}) \quad \text { in } \mathbb{R} \times \bar{\Omega}, \\
\nu A \nabla \bar{U} \geq 0 \quad \text { on } \mathbb{R} \times \partial \Omega
\end{array}\right.
$$

and

$$
\left\{\begin{array}{l}
\underline{U}_{t}-\nabla \cdot(A(x, y) \nabla \underline{U})+q(x, y) \cdot \nabla \underline{U} \leq f(x, y, \underline{U}) \quad \text { in } \mathbb{R} \times \bar{\Omega}, \\
v A \nabla \underline{U} \leq 0 \quad \text { on } \mathbb{R} \times \partial \Omega,
\end{array}\right.
$$

such that $\bar{U}<p^{+}$and $\underline{U}<p^{+}$in $\mathbb{R} \times \bar{\Omega}$. Assume that $\bar{U}(t, x, y)=\bar{\Phi}(x \cdot e-c t, x, y)$ and $\underline{U}(t, x, y)=\underline{\Phi}(x \cdot e-c t, x, y)$, where $\bar{\Phi}$ and $\underline{\Phi}$ are periodic in $(x, y), c \neq 0$ and $e \in \overline{\mathbb{R}}^{d} \times\{0\}^{N-d}$ with $|e|=1$. If there exists $h \in \mathbb{R}$ such that

$$
\left\{\begin{array}{l}
\bar{\Phi}(s, x, y)>p^{+}(x, y)-\rho \quad \text { for all } s \leq h \text { and }(x, y) \in \bar{\Omega}, \\
\bar{\Phi}(h, x, y) \geq \underline{\Phi}(h, x, y) \quad \text { for all }(x, y) \in \bar{\Omega}, \\
\liminf _{s \rightarrow-\infty}\left[\min _{(x, y) \in \bar{\Omega}}(\bar{\Phi}(s, x, y)-\underline{\Phi}(s, x, y))\right] \geq 0,
\end{array}\right.
$$

then

$$
\bar{\Phi}(s, x, y) \geq \underline{\Phi}(s, x, y) \quad \text { for all } s \leq h \text { and }(x, y) \in \bar{\Omega},
$$

that is, $\bar{U}(t, x, y) \geq \underline{U}(t, x, y)$ for all $(t, x, y) \in \mathbb{R} \times \bar{\Omega}$ such that $x \cdot e-c t \leq h$.

We then use the following general uniqueness result, which does not require the KPP assumption (1.6):

Theorem 2.2. Let e be a unit vector in $\mathbb{R}^{d} \times\{0\}^{N-d}$ and $c \in \mathbb{R}^{*}$ be given. Assume that for any two pulsating traveling fronts $U(t, x, y)=\phi(x \cdot e-c t, x, y)$ and $U^{\prime}(t, x, y)=$ $\phi^{\prime}(x \cdot e-c t, x, y)$ in the sense of $[1.7)$, there exists a constant $C_{\left[\phi, \phi^{\prime}\right]} \in(0,+\infty)$ such that

$$
\frac{\phi(s, x, y)-p^{-}(x, y)}{\phi^{\prime}(s, x, y)-p^{-}(x, y)} \rightarrow C_{\left[\phi, \phi^{\prime}\right]} \quad \text { as } s \rightarrow+\infty \text { uniformly in }(x, y) \in \bar{\Omega}
$$


Then, if $U_{1}(t, x, y)=\phi_{1}(x \cdot e-c t, x, y)$ and $U_{2}(t, x, y)=\phi_{2}(x \cdot e-c t, x, y)$ are two pulsating fronts, there exists $\sigma \in \mathbb{R}$ such that 1.8) and (1.9) hold.

Proof. Step 1. Let $U(t, x, y)=\phi(x \cdot e-c t, x, y)$ be any pulsating traveling front in the sense of (1.7). From Proposition 2.2 of [20], we know that there exist two positive real numbers $\lambda_{m, \phi} \leq \lambda_{M, \phi}$ such that

$$
\left\{\begin{array}{l}
\lambda_{m, \phi}:=\liminf _{s \rightarrow+\infty}\left(\min _{(x, y) \in \bar{\Omega}} \frac{-\phi_{s}(s, x, y)}{\phi(s, x, y)-p^{-}(x, y)}\right)>0, \\
\lambda_{M, \phi}:=\limsup _{s \rightarrow+\infty}\left(\max _{(x, y) \in \bar{\Omega}} \frac{-\phi_{s}(s, x, y)}{\phi(s, x, y)-p^{-}(x, y)}\right)<+\infty .
\end{array}\right.
$$

For each $\sigma \in \mathbb{R}$, denote by $C_{\left[\phi^{\sigma}, \phi\right]}$ the constant defined as in the statement of Theorem 2.2, with

$$
\phi^{\sigma}(\cdot, \cdot, \cdot):=\phi(\cdot+\sigma, \cdot, \cdot) .
$$

Then we claim that

$$
\exists v>0, \forall \sigma \in \mathbb{R}, \quad C_{\left[\phi^{\sigma}, \phi\right]}=e^{-v \sigma} .
$$

Indeed, for any $\sigma, \sigma^{\prime} \in \mathbb{R}$ and $(x, y) \in \bar{\Omega}$,

$$
\begin{aligned}
C_{\left[\phi^{\left.\sigma+\sigma^{\prime}, \phi\right]}\right.} & =\lim _{s \rightarrow+\infty} \frac{\phi\left(s+\sigma+\sigma^{\prime}, x, y\right)-p^{-}(x, y)}{\phi(s, x, y)-p^{-}(x, y)} \\
& =\lim _{s \rightarrow+\infty}\left(\frac{\phi\left(s+\sigma+\sigma^{\prime}, x, y\right)-p^{-}(x, y)}{\phi\left(s+\sigma^{\prime}, x, y\right)-p^{-}(x, y)} \frac{\phi\left(s+\sigma^{\prime}, x, y\right)-p^{-}(x, y)}{\phi(s, x, y)-p^{-}(x, y)}\right) \\
& =C_{\left[\phi^{\sigma}, \phi\right]} C_{\left[\phi^{\sigma^{\prime}}, \phi\right]} .
\end{aligned}
$$

Furthermore, the function $\sigma \mapsto C_{\left[\phi^{\sigma}, \phi\right]}$ is nonincreasing in $\mathbb{R}$ since $\phi(s, x, y)$ is decreasing in $s$ (see Proposition 2.5 in [20]). As a consequence, there exists $v \in[0,+\infty)$ such that $C_{\left[\phi^{\sigma}, \phi\right]}=e^{-v \sigma}$ for all $\sigma \in \mathbb{R}$. Using 2.2 , we finally obtain $v \in\left[\lambda_{m, \phi}, \lambda_{M, \phi}\right]$, whence $v>0$. This shows 2.3.

Step 2. Now, let $U_{1}(t, x, y)=\phi_{1}(x \cdot e-c t, x, y)$ and $U_{2}(t, x, y)=\phi_{2}(x \cdot e-c t, x, y)$ be two pulsating fronts satisfying (1.7). From (2.3) applied with $\phi=\phi_{1}$, we know that, for $\sigma<0$ negative enough,

$$
C_{\left[\phi_{1}^{\sigma}, \phi_{2}\right]}=C_{\left[\phi_{1}^{\sigma}, \phi_{1}\right]} C_{\left[\phi_{1}, \phi_{2}\right]}>1 .
$$

Since $\phi_{1}$ is strictly decreasing with respect to $s$, we deduce that there exist $\Sigma_{0}>0, \sigma_{0}<0$ such that

$$
\forall \sigma \leq \sigma_{0}, \quad \phi_{2} \leq \phi_{1}^{\sigma} \quad \text { in }\left[\Sigma_{0},+\infty\right) \times \bar{\Omega} .
$$

Since $\phi_{1}(-\infty, \cdot, \cdot)=p^{+}$, decreasing $\sigma_{0}$ if necessary, one can assume that

$$
\phi_{1}^{\sigma}>p^{+}-\rho \quad \text { in }\left(-\infty, \Sigma_{0}\right] \times \bar{\Omega} \text { for all } \sigma \leq \sigma_{0} .
$$

All assumptions of Lemma 2.1 are then satisfied, for all $\sigma \leq \sigma_{0}$, with

$$
\bar{U}(t, x, y)=U_{1}(t-\sigma / c, x, y), \quad \underline{U}=U_{2}, \quad \bar{\Phi}=\phi_{1}^{\sigma}, \quad \underline{\Phi}=\phi_{2}, \quad h=\Sigma_{0} .
$$


As a consequence, $\phi_{2} \leq \phi_{1}^{\sigma}$ in $\left(-\infty, \Sigma_{0}\right] \times \bar{\Omega}$ for all $\sigma \leq \sigma_{0}$ and, from 2.4 , we finally get

$$
\phi_{2} \leq \phi_{1}^{\sigma} \quad \text { in } \mathbb{R} \times \bar{\Omega} \text { for all } \sigma \leq \sigma_{0}
$$

Let us set

$$
\sigma^{*}=\sup \left\{\sigma \in \mathbb{R}: \phi_{2} \leq \phi_{1}^{\sigma} \text { in } \mathbb{R} \times \bar{\Omega}\right\} .
$$

Observe that $\sigma^{*} \geq \sigma_{0}$. Since $\phi_{1}(+\infty, \cdot, \cdot)=p^{-}$and $\phi_{2}(s, x, y)>p^{-}(x, y)$ for all $(s, x, y) \in \mathbb{R} \times \bar{\Omega}$, we also know that $\sigma^{*}<+\infty$. Moreover, $\phi_{2} \leq \phi_{1}^{\sigma^{*}}$ in $\mathbb{R} \times \bar{\Omega}$. Set

$$
z(s, x, y)=\phi_{1}^{\sigma^{*}}(s, x, y)-\phi_{2}(s, x, y) .
$$

The function $z$ is continuous in $(s, x, y)$, periodic in $(x, y)$ and nonnegative. In particular, the minimum of $z$ over all sets of the type $[-\Sigma, \Sigma] \times \bar{\Omega}$, with $\Sigma>0$, is reached and it is either positive or zero.

Case 1: Assume that there exists $\Sigma>0$ such that $\min _{(s, x, y) \in[-\Sigma, \Sigma] \times \bar{\Omega}} z(s, x, y)=0$. The function

$$
v(t, x, y):=z(x \cdot e-c t, x, y)
$$

is nonnegative in $\mathbb{R} \times \bar{\Omega}$ and it vanishes at a point $\left(t^{*}, x^{*}, y^{*}\right)$ such that $\left|x^{*} \cdot e-c t^{*}\right| \leq \Sigma$. Moreover, it satisfies the boundary condition $v A(x, y) \nabla v=0$ on $\mathbb{R} \times \partial \Omega$, and the equation

$$
v_{t}-\nabla \cdot(A \nabla v)+q \cdot \nabla v=f\left(x, y, U_{1}\left(t-\sigma^{*} / c, x, y\right)\right)-f\left(x, y, U_{2}(t, x, y)\right)
$$

in $\mathbb{R} \times \bar{\Omega}$. Since $f$ is globally Lipschitz-continuous in $\bar{\Omega} \times \mathbb{R}$, there exists a bounded function $b(t, x, y)$ such that

$$
v_{t}-\nabla \cdot(A \nabla v)+q \cdot \nabla v+b v=0 \quad \text { for all }(t, x, y) \in \mathbb{R} \times \bar{\Omega} .
$$

From the strong maximum principle and Hopf lemma, the function $v$ is then identically 0 in $\left(-\infty, t^{*}\right] \times \bar{\Omega}$, and then in $\mathbb{R} \times \bar{\Omega}$ by uniqueness of the Cauchy problem associated to 2.5 . We thus obtain $z \equiv 0$, that is, $\phi_{2} \equiv \phi_{1}^{\sigma^{*}}$ in $\mathbb{R} \times \bar{\Omega}$.

Case 2: Assume that, for all $\Sigma>0, \min _{(s, x, y) \in[-\Sigma, \Sigma] \times \bar{\Omega}} z(s, x, y)>0$. The function $z$ is uniformly continuous in $\mathbb{R} \times \bar{\Omega}$, thus, for all $\Sigma>0$, there exists $\sigma_{\Sigma} \in\left(\sigma^{*}, \sigma^{*}+1\right)$ such that

$$
\phi_{2} \leq \phi_{1}^{\sigma} \quad \text { in }[-\Sigma, \Sigma] \times \bar{\Omega} \text { for all } \sigma \in\left[\sigma^{*}, \sigma_{\Sigma}\right]
$$

For $\Sigma$ large enough,

$$
\phi_{1}^{\sigma_{\Sigma}}>p^{+}-\rho \quad \text { in }(-\infty,-\Sigma] \times \bar{\Omega} .
$$

Moreover, $\phi_{1}^{\sigma_{\Sigma}}(-\Sigma, x, y) \geq \phi_{2}(-\Sigma, x, y)$ in $\bar{\Omega}$ from 2.6. Applying Lemma 2.1 with

$$
\bar{U}(t, x, y)=U_{1}\left(t-\sigma_{\Sigma} / c, x, y\right), \quad \underline{U}=U_{2}, \quad \bar{\Phi}=\phi_{1}^{\sigma_{\Sigma}}, \quad \underline{\Phi}=\phi_{2}, \quad h=-\Sigma,
$$

we get $\phi_{2} \leq \phi_{1}^{\sigma_{\Sigma}}$ in $(-\infty,-\Sigma] \times \bar{\Omega}$. Together with $\left[2.6\right.$, since $\phi_{1}$ is decreasing in $s$, it follows that

$\exists \Sigma_{1}>0, \forall \Sigma \geq \Sigma_{1}, \exists \sigma_{\Sigma}>\sigma^{*}, \forall \sigma \in\left[\sigma^{*}, \sigma_{\Sigma}\right], \quad \phi_{2} \leq \phi_{1}^{\sigma} \quad$ in $(-\infty, \Sigma] \times \bar{\Omega}$. 
Assume now that

$$
\exists \varepsilon>0, \exists \widetilde{\Sigma}>0, \quad \phi_{1}^{\sigma^{*}}-\phi_{2} \geq \varepsilon\left(\phi_{2}-p^{-}\right) \quad \text { in }[\widetilde{\Sigma},+\infty) \times \bar{\Omega} .
$$

Let $\left(\sigma_{n}\right)_{n \in \mathbb{N}}$ be a decreasing sequence with $\lim _{n \rightarrow+\infty} \sigma_{n}=\sigma^{*}$. In particular, $\sigma_{n}>\sigma^{*}$ for all $n \in \mathbb{N}$. Divide $\sqrt{2.8}$ by $\phi_{1}^{\sigma_{n}}-p^{-}$. We get, for all $(s, x, y) \in(-\infty,-\widetilde{\Sigma}] \times \bar{\Omega}$,

$$
\frac{\phi_{1}^{\sigma^{*}}(s, x, y)-p^{-}(x, y)}{\phi_{1}^{\sigma_{n}}(s, x, y)-p^{-}(x, y)}-\frac{\phi_{2}(s, x, y)-p^{-}(x, y)}{\phi_{1}^{\sigma_{n}}(s, x, y)-p^{-}(x, y)} \geq \varepsilon \frac{\phi_{2}(s, x, y)-p^{-}(x, y)}{\phi_{1}^{\sigma_{n}}(s, x, y)-p^{-}(x, y)} .
$$

Passing to the limit as $s \rightarrow+\infty$, we obtain $C_{\left[\phi_{1}^{\sigma^{*}}, \phi_{1}^{\sigma_{n}}\right]}-C_{\left[\phi_{2}, \phi_{1}^{\sigma_{n}}\right]} \geq \varepsilon C_{\left[\phi_{2}, \phi_{1}^{\sigma_{n}}\right]}$, or, equivalently,

$$
\frac{1}{1+\varepsilon} C_{\left[\phi_{1}^{\sigma^{*}-\sigma_{n}}, \phi_{1}\right]} \geq C_{\left[\phi_{2}, \phi_{1}^{\sigma_{n}}\right]} .
$$

But, from 2.3 applied with $\phi=\phi_{1}$, we know that, for $n$ large enough, $C_{\left[\phi_{1}^{\sigma^{*}-\sigma_{n}}, \phi_{1}\right]}<$ $1+\varepsilon$, whence $C_{\left[\phi_{2}, \phi_{1}^{\left.\sigma_{n}\right]}\right.}<1$. As a consequence, there exist $n_{1} \in \mathbb{N}$ and $\Sigma_{2}>\widetilde{\Sigma}$ such that $\phi_{2} \leq \phi_{1}^{\sigma_{n_{1}}}$ in $\left[\Sigma_{2},+\infty\right) \times \bar{\Omega}$, and therefore,

$$
\phi_{2} \leq \phi_{1}^{\sigma} \quad \text { in }\left[\Sigma_{2},+\infty\right) \times \bar{\Omega} \text { for all } \sigma \in\left[\sigma^{*}, \sigma_{n_{1}}\right]
$$

Denote $\bar{\Sigma}:=\max \left\{\Sigma_{1}, \Sigma_{2}\right\}$ and $\bar{\sigma}:=\min \left\{\sigma_{n_{1}}, \sigma_{\bar{\Sigma}}\right\}$, where $\sigma_{\bar{\Sigma}}$ is defined by 2.7 . From 2.7 and 2.9, we obtain $\phi_{2} \leq \phi_{1}^{\bar{\sigma}}$ in $\mathbb{R} \times \bar{\Omega}$, which contradicts the definition of $\sigma^{*}$, since $\bar{\sigma}>\sigma^{*}$. Therefore, the property 2.8 cannot hold.

Finally, we obtain the existence of a real number $\sigma^{*}$ such that $\phi_{1}^{\sigma^{*}} \geq \phi_{2}$ and either

- $\phi_{1}^{\sigma^{*}} \equiv \phi_{2}$, or

- the property 2.8 is false, thus there exists a sequence $\left(s_{n}, x_{n}, y_{n}\right)_{n \in \mathbb{N}}$ in $\mathbb{R} \times \bar{\Omega}$ such that $\lim _{n \rightarrow+\infty} s_{n}=+\infty$ and

$$
0 \leq \phi_{1}^{\sigma^{*}}\left(s_{n}, x_{n}, y_{n}\right)-\phi_{2}\left(s_{n}, x_{n}, y_{n}\right) \leq \frac{\phi_{2}\left(s_{n}, x_{n}, y_{n}\right)-p^{-}\left(x_{n}, y_{n}\right)}{n} \quad \text { for all } n \in \mathbb{N} \text {. }
$$

Since $\phi_{1}$ and $\phi_{2}$ were chosen arbitrarily, we also obtain the existence of a real number $-\sigma_{*}$ such that $\phi_{2}^{-\sigma_{*}} \geq \phi_{1}$ and either

- $\phi_{2}^{-\sigma_{*}} \equiv \phi_{1}$, or

- there exists a sequence $\left(s_{n}^{\prime}, x_{n}^{\prime}, y_{n}^{\prime}\right)_{n \in \mathbb{N}}$ in $\mathbb{R} \times \bar{\Omega}$ such that $\lim _{n \rightarrow+\infty} s_{n}^{\prime}=+\infty$ and $0 \leq \phi_{2}^{-\sigma_{*}}\left(s_{n}^{\prime}, x_{n}^{\prime}, y_{n}^{\prime}\right)-\phi_{1}\left(s_{n}^{\prime}, x_{n}^{\prime}, y_{n}^{\prime}\right) \leq \frac{\phi_{1}\left(s_{n}^{\prime}, x_{n}^{\prime}, y_{n}^{\prime}\right)-p^{-}\left(x_{n}^{\prime}, y_{n}^{\prime}\right)}{n} \quad$ for all $n \in \mathbb{N}$.

Equivalently, setting $s_{n}^{\prime \prime}=s_{n}^{\prime}-\sigma_{*}$, we get, for all $n \in \mathbb{N}$,

$$
0 \leq \phi_{2}\left(s_{n}^{\prime \prime}, x_{n}^{\prime}, y_{n}^{\prime}\right)-\phi_{1}^{\sigma_{*}}\left(s_{n}^{\prime \prime}, x_{n}^{\prime}, y_{n}^{\prime}\right) \leq \frac{\phi_{1}^{\sigma_{*}}\left(s_{n}^{\prime \prime}, x_{n}^{\prime}, y_{n}^{\prime}\right)-p^{-}\left(x_{n}^{\prime}, y_{n}^{\prime}\right)}{n} .
$$


Eventually, either the property 1.8 of Theorem 2.2 holds for some $\sigma \in \mathbb{R}$, or there exist $\sigma^{*}, \sigma_{*} \in \mathbb{R}$ such that $\phi_{1}^{\sigma^{*}} \geq \phi_{2}$ and $\phi_{2}^{-\sigma_{*}} \geq \phi_{1}$ (that is, $\phi_{2} \geq \phi_{1}^{\sigma_{*}}$ ) in $\mathbb{R} \times \bar{\Omega}$, and properties (2.10) and 2.11) hold true. Divide the inequalities in 2.10) and (2.11) by $\phi_{1}\left(s_{n}, x_{n}, y_{n}\right)-p^{-}\left(x_{n}, y_{n}\right)$ and $\phi_{1}\left(s_{n}^{\prime \prime}, x_{n}^{\prime}, y_{n}^{\prime}\right)-p^{-}\left(x_{n}^{\prime}, y_{n}^{\prime}\right)$ respectively, and pass to the limit as $n \rightarrow+\infty$. It follows that

$$
C_{\left[\phi_{1}^{\sigma^{*}}, \phi_{1}\right]}=C_{\left[\phi_{2}, \phi_{1}\right]} \quad \text { and } \quad C_{\left[\phi_{2}, \phi_{1}\right]}=C_{\left[\phi_{1}^{\sigma_{*}}, \phi_{1}\right]} \cdot
$$

Thus, $C_{\left[\phi_{1}^{\sigma^{*}}, \phi_{1}\right]}=C_{\left[\phi_{1}^{\sigma_{*}}, \phi_{1}\right]}$. From 2.3 applied with $\phi=\phi_{1}$, we conclude that $\sigma^{*}=$ $\sigma_{*}=: \sigma$. Since $\phi_{1}^{\sigma_{*}} \leq \phi_{2} \leq \phi_{1}^{\sigma^{*}}$, we finally get $\phi_{1}^{\sigma} \equiv \phi_{2}$. Property 1.8 has been shown.

The assumption (2.1) in Theorem 2.2 says that, for a given speed $c$, any two pulsating traveling fronts have the same asymptotic behavior, up to multiplicative constants, as $s \rightarrow+\infty$, that is, as they approach the unstable state $p^{-}$. This condition is essential and it is known to be fulfilled for instance in simplified situations, like in space-homogeneous settings or in straight infinite cylinders with shear flows, that is, for problems (1.27) and (1.28). In our general periodic setting, property (2.1) is a reasonable conjecture but it has not been shown yet in general. However, in the KPP case (1.6), this property is satisfied and the proof of Theorem 1.1 follows:

Proof of Theorem 1.1. Under the KPP assumption (1.6), the hypothesis 2.1) in Theorem 2.2 is automatically satisfied, because of formulas $(1.22)$ and $(1.23)$ (see Theorem 1.3 in [20]). As a consequence, (1.8) and (1.9) follow immediately.

\section{Stability of monostable fronts with speeds $c>c^{*}(e)$}

This section is devoted to the proof of Theorem 1.3 . The general strategy is based on the construction of suitable sub- and super-solutions which trap the solution $u$ of the Cauchy problem (1.1) and which can eventually be chosen as close as we want to the front $U$ as $t \rightarrow+\infty$. The sub- and super-solutions are close to the pulsating front $U$, up to some phase-shifts and exponentially small correcting terms (see Proposition 3.2 below in Subsection 3.2). Furthermore, more precise exponential estimates are established in the region where $s$ is large (see Proposition 3.3. In Subsection 3.3. we prove a Liouville type result: any time-global solution which satisfies the same type of exponential estimates as in Proposition 3.3 must be a pulsating front (see Proposition 3.4. In Subsection 3.4, we complete the proof of Theorem 1.3 , by arguing by contradiction and using the estimates of Subsection 3.2 and the aforementioned Liouville type result.

Due to the generality of the framework and the assumptions, the proof is rather involved and requires many technicalities. Before entering into the core of the proof, we introduce a few notations. 


\subsection{Preliminary notations}

We assume here that $\mu^{+}>0$ and that

$$
U(t, x, y)=\phi(x \cdot e-c t, x, y)
$$

is a pulsating traveling front with speed $c>c^{*}(e)$ satisfying 1.18).

Recall that $k(0)=\mu^{-}<0$ and that $\lambda_{c}>0$ is given by 1.16 . By continuity of $k$, there exists $\lambda>\lambda_{c}$ such that

$$
-k(\lambda) / \lambda<c=-k\left(\lambda_{c}\right) / \lambda_{c}
$$

and

$$
k(\lambda)+\lambda c \leq \mu^{+}
$$

Define $\omega>0$ by

$$
k(\lambda)+\lambda c=2 \omega .
$$

Let $\theta$ be a $C^{2}(\bar{\Omega})$ nonpositive periodic function such that

$$
\nu A \nabla \theta+\nu A e=0 \quad \text { on } \partial \Omega .
$$

For instance, up to a constant, $\theta$ can be chosen to be a minimizer in $H_{\text {per }}^{1}$ of the functional

$$
\varphi \mapsto \int_{\Omega} \nabla \varphi A \nabla \varphi+2 \int_{\partial \Omega}(\nu A e) \varphi,
$$

where $H_{\text {per }}^{1}$ denotes the set of periodic functions in $\Omega$ which are in $H_{\text {loc }}^{1}(\bar{\Omega})$. Let $\psi^{+}$be given by (1.17) and $\psi=\psi_{\lambda}$ denote the positive principal eigenvalue of the operator $L_{\lambda}$, given in 1.12$)$, such that $\|\psi\|_{L^{\infty}(\Omega)}=1$. Set $m^{+}=\min _{\bar{\Omega}} \psi^{+}>0$ and let $\underline{s} \in \mathbb{R}$ be such that

$$
e^{-\lambda(\underline{s}-1)} \leq m^{+}
$$

Let $\chi$ be a $C^{2}(\mathbb{R} ;[0,1])$ function such that

$$
\chi^{\prime}(s) \geq 0 \text { for all } s \in \mathbb{R}, \quad \chi(s)=0 \text { for } s \leq \underline{s}-1, \quad \chi(s)=1 \text { for } s \geq \underline{s} .
$$

Let $g$ be the function defined for all $(s, x, y) \in \mathbb{R} \times \bar{\Omega}$ by

$$
g(s, x, y)=\psi(x, y) e^{-\lambda s} \chi(s+\theta(x, y))+\psi^{+}(x, y)(1-\chi(s+\theta(x, y))) .
$$

Observe that $g$ is nonnegative, bounded and periodic with respect to $(x, y)$ in $\mathbb{R} \times \bar{\Omega}$.

Lemma 3.1. Define

$$
\rho^{+}=\min _{\bar{\Omega}} \frac{p^{+}-p^{-}}{\psi^{+}}>0
$$

Then

$$
\limsup _{\varsigma \rightarrow-\infty} \sup _{(s, x, y) \in \mathbb{R} \times \bar{\Omega}, \rho \in\left(0, \rho^{+}\right]} \frac{\phi(s, x, y)-\rho g(s+\varsigma, x, y)-p^{+}(x, y)}{\rho \psi^{+}(x, y)} \leq-1 .
$$


Proof. Assume the conclusion does not hold. Then there exist $0<\varepsilon \leq 1$ and sequences $\left(s_{n}, x_{n}, y_{n}\right)_{n \in \mathbb{N}}$ in $\mathbb{R} \times \bar{\Omega},\left(\rho_{n}^{\prime}\right)_{n \in \mathbb{N}}$ in $\left(0, \rho^{+}\right]$and $\left(\varsigma_{n}\right)_{n \in \mathbb{N}}$ such that $\lim _{n \rightarrow+\infty} \varsigma_{n}=-\infty$ and

$$
\frac{\phi\left(s_{n}, x_{n}, y_{n}\right)-\rho_{n}^{\prime} g\left(s_{n}+\varsigma_{n}, x_{n}, y_{n}\right)-p^{+}\left(x_{n}, y_{n}\right)}{\rho_{n}^{\prime} \psi^{+}\left(x_{n}, y_{n}\right)} \geq-1+\varepsilon
$$

for all $n \in \mathbb{N}$. Up to taking a subsequence, either the sequence $\left(s_{n}+\varsigma_{n}\right)_{n \in \mathbb{N}}$ converges to $-\infty$ as $n \rightarrow+\infty$, or it is bounded from below. In the first case, and since $\phi \leq p^{+}$, one has

$$
\frac{-g\left(s_{n}+\varsigma_{n}, x_{n}, y_{n}\right)}{\psi^{+}\left(x_{n}, y_{n}\right)} \geq-1+\varepsilon
$$

The passage to the limit as $n \rightarrow+\infty$ leads to $-1 \geq-1+\varepsilon$ by definition of $g$, which is impossible. Thus, the sequence $\left(s_{n}+s_{n}\right)_{n \in \mathbb{N}}$ is bounded from below, whence $\lim _{n \rightarrow+\infty} s_{n}=+\infty$. Since $g \geq 0$ and $\rho_{n}^{\prime} \leq \rho^{+}$, one gets

$$
\frac{\phi\left(s_{n}, x_{n}, y_{n}\right)-p^{+}\left(x_{n}, y_{n}\right)}{\psi^{+}\left(x_{n}, y_{n}\right)} \geq-(1-\varepsilon) \rho_{n}^{\prime} \geq-(1-\varepsilon) \rho^{+}>-\rho^{+} .
$$

Since all functions $\phi, p^{+}$and $\psi^{+}$are periodic in $(x, y)$, one can assume that $\left(x_{n}, y_{n}\right) \rightarrow$ $\left(x_{\infty}, y_{\infty}\right) \in \bar{\Omega}$ as $n \rightarrow+\infty$ (up to taking another subsequence). The limit as $n \rightarrow+\infty$ in 3.8 leads to

$$
\frac{p^{-}\left(x_{\infty}, y_{\infty}\right)-p^{+}\left(x_{\infty}, y_{\infty}\right)}{\psi^{+}\left(x_{\infty}, y_{\infty}\right)}>-\rho^{+},
$$

which is ruled out by 3.7. As a consequence, Lemma 3.1 has been proved.

In what follows, we fix $s_{0} \leq 0$ such that

$\forall \rho \in\left(0, \rho^{+}\right], \forall(s, x, y) \in \mathbb{R} \times \bar{\Omega}, \quad \frac{\phi(s, x, y)-\rho g\left(s+s_{0}, x, y\right)-p^{+}(x, y)}{\psi^{+}(x, y)} \leq-\frac{\rho}{2}$.

Set, for all $(s, x, y) \in \mathbb{R} \times \bar{\Omega} \unlhd$

$$
\left\{\begin{aligned}
B(s, x, y)= & \left(\zeta^{-}+\omega\right) \psi e^{-\lambda s} \chi(s+\theta)+\left(\zeta^{+}+\mu^{+}-\omega\right) \psi^{+}(1-\chi(s+\theta)) \\
& +\left\{\left(\psi e^{-\lambda s}-\psi^{+}\right) \times[c+q \cdot(\nabla \theta+e)-\nabla \cdot(A \nabla \theta+A e)]\right. \\
& \left.+2\left(-\lambda \psi e^{-\lambda s} e-e^{-\lambda s} \nabla \psi+\nabla \psi^{+}\right) A(\nabla \theta+e)\right\} \chi^{\prime}(s+\theta) \\
& -\left(\psi e^{-\lambda s}-\psi^{+}\right)(\nabla \theta A \nabla \theta+e A e+2 e A \nabla \theta) \chi^{\prime \prime}(s+\theta) \\
C(s, x, y)= & -\lambda \psi e^{-\lambda s} \chi(s+\theta)+\left(\psi e^{-\lambda s}-\psi^{+}\right) \chi^{\prime}(s+\theta),
\end{aligned}\right.
$$

where all functions $A, q, \zeta^{ \pm}, \psi, \psi^{+}, \theta$ are evaluated at $(x, y)$, and $\zeta^{ \pm}(x, y)=$ $\frac{\partial f}{\partial u}\left(x, y, p^{ \pm}(x, y)\right)$. Let us check that the function $C$ is nonpositive. To see this, since $\lambda \psi \chi \geq 0$ and $\chi^{\prime} \geq 0$, one only needs to check that $\psi(x, y) e^{-\lambda s}-\psi^{+} \leq 0$ when $\chi^{\prime}(s+\theta(x, y))>0$. If $\chi^{\prime}(s+\theta(x, y))>0$, then $s+\theta(x, y) \geq \underline{s}-1$, whence $s \geq$

\footnotetext{
4 In formula 3.10 , when the letter $e$ is alone, it means the direction $e$, while $e^{-\lambda s}$ means
} $\exp (-\lambda s)$. 
$\underline{s}-1-\theta(x, y) \geq \underline{s}-1(\theta$ is nonpositive $)$ and $\psi(x, y) e^{-\lambda s} \leq e^{-\lambda(\underline{s}-1)} \leq m^{+} \leq \psi^{+}(x, y)$ from (3.5). Therefore,

$$
C(s, x, y) \leq 0 \quad \text { for all }(s, x, y) \in \mathbb{R} \times \bar{\Omega} .
$$

Now, choose $\rho^{-}>0$ such that

$$
\forall(x, y, \rho) \in \bar{\Omega} \times\left[0, \rho^{-}\right], \quad\left|\frac{\partial f}{\partial u}\left(x, y, p^{-}(x, y)+\rho\right)-\zeta^{-}(x, y)\right| \leq \omega .
$$

Recall that $\phi_{s}<0$ in $\mathbb{R} \times \bar{\Omega}$ and notice that, because of $(1.18, \sqrt{2.2})$ and $\lambda>\lambda_{c}$,

$$
\sup _{(x, y) \in \bar{\Omega}} \frac{\left|C\left(s+s_{0}, x, y\right)\right|}{\left|\phi_{s}(s, x, y)\right|} \rightarrow 0 \quad \text { as } s \rightarrow+\infty .
$$

Owing to the definitions of the functions $B$ and $C$, there exists $s^{+} \geq 0$ such that

$\forall(s, x, y) \in\left[s^{+},+\infty\right) \times \bar{\Omega},\left\{\begin{array}{l}p^{-}(x, y)<\phi(s, x, y) \leq p^{-}(x, y)+\rho^{-} / 2, \\ g\left(s+s_{0}, x, y\right)=\psi(x, y) e^{-\lambda\left(s+s_{0}\right)} \leq \rho^{-} / 2, \\ B\left(s+s_{0}, x, y\right)=\left(\zeta^{-}(x, y)+\omega\right) g\left(s+s_{0}, x, y\right), \\ C\left(s+s_{0}, x, y\right)=-\lambda \psi(x, y) e^{-\lambda\left(s+s_{0}\right)}<0, \\ -\phi_{s}(s, x, y)+\rho^{+} C\left(s+s_{0}, x, y\right) \geq 0 .\end{array}\right.$

As above, one can choose $\rho_{1}^{+} \in\left(0, \rho^{+}\right]$such that

$$
\forall(x, y, \rho) \in \bar{\Omega} \times\left[0, \rho_{1}^{+}\right], \quad\left|\frac{\partial f}{\partial u}\left(x, y, p^{+}(x, y)-\rho \psi^{+}(x, y)\right)-\zeta^{+}(x, y)\right| \leq \omega .
$$

Since $\min _{\bar{\Omega}} \psi^{+}>0$, there exists $s^{-} \leq 0$ such that

$\forall(s, x, y) \in\left(-\infty, s^{-}\right] \times \bar{\Omega},\left\{\begin{array}{l}p^{+}(x, y)-\frac{\rho_{1}^{+}}{2} \psi^{+}(x, y) \leq \phi(s, x, y)<p^{+}(x, y), \\ g(s, x, y)=\psi^{+}(x, y), \\ B(s, x, y)=\left(\zeta^{+}(x, y)+\mu^{+}-\omega\right) \psi^{+}(x, y), \\ C(s, x, y)=0 .\end{array}\right.$

Once the real numbers $s^{ \pm}$have been chosen, let $\delta$ be given by

$$
\delta=\min _{s^{-} \leq s \leq s^{+},(x, y) \in \bar{\Omega}}\left(-\phi_{s}(s, x, y)\right) .
$$

The real number $\delta$ is positive since the function $\phi_{s}$ is continuous, negative and periodic with respect to $(x, y)$ in $\mathbb{R} \times \bar{\Omega}$. Define

$$
\begin{aligned}
& \varepsilon_{1}=\min \left(\frac{\rho_{1}^{+}}{4}, \frac{\delta}{4\|C\|_{\infty}}\right)>0, \\
& \varepsilon_{0}=m^{+} \varepsilon_{1}>0,
\end{aligned}
$$

where $m^{+}=\min _{\bar{\Omega}} \psi^{+}>0$. 
Lastly, since the function $\partial f / \partial u$ is continuous in $\bar{\Omega} \times \mathbb{R}$ and periodic with respect to $(x, y)$, the quantity

$$
M=\max _{(x, y) \in \bar{\Omega}, p^{-}(x, y) \leq u \leq p^{+}(x, y)}\left|\frac{\partial f}{\partial u}(x, y, u)\right|
$$

is finite. Notice also that all functions $g, B$ and $C$ are bounded in $\mathbb{R} \times \bar{\Omega}$. Let $\underline{\sigma}$ be the nonnegative real number defined by

$$
\underline{\sigma}=\max \left(\frac{M\|g\|_{\infty}+\|B\|_{\infty}}{\omega\|C\|_{\infty}}, \frac{M\|g\|_{\infty}+\|B\|_{\infty}}{\omega \delta}\right)
$$

\subsection{Sub- and super-solutions}

The method which is used to prove the convergence of $u(t, x, y)$ to the pulsating front $U(t, x, y)$ is first based on the construction of suitable sub- and super-solutions which converge to finite shifts of the front $\phi$ as $t \rightarrow+\infty$. This idea is inspired by a paper by Fife and McLeod [15] devoted to one-dimensional bistable equations. The method has to be adapted here to the periodic framework and to monostable equations. Then we will prove that the shifts can be as small as we want as $x \cdot e-c t \rightarrow+\infty$. These comparisons will be used in the following subsection to prove the uniform convergence of $u$ to the front $U$ as $t \rightarrow+\infty$, without shift.

We assume that $\mu^{+}>0$ and that $U(t, x, y)=\phi(x \cdot e-c t, x, y)$ is a pulsating traveling front with speed $c>c^{*}(e)$ and satisfying (1.18). We use the notations of the previous section and we assume that the initial condition $u_{0}$ satisfies 1.19$]$ and 1.20 . In what follows, for all $\kappa \in \mathbb{R}$ and $(t, x, y) \in \mathbb{R} \times \bar{\Omega}$, we denote

$$
s_{\kappa}(t, x)=x \cdot e-c t+\kappa-\kappa e^{-\omega t} .
$$

Proposition 3.2. Under all assumptions of Theorem 1.3 and with the above notations, there exist $t_{0}>0$ and $\sigma_{0} \geq \underline{\sigma}$ such that

$$
\begin{aligned}
& \max \left[\phi\left(s_{\sigma_{0}}(t, x), x, y\right)-2 \varepsilon_{1} g\left(s_{\sigma_{0}}(t, x)+s_{0}, x, y\right) e^{-\omega t}, p^{-}(x, y)\right] \\
& \quad \leq u(t, x, y) \leq \min \left[\phi\left(s_{-\sigma_{0}}(t, x), x, y\right)+g\left(s_{-\sigma_{0}}(t, x), x, y\right) e^{-\omega t}, p^{+}(x, y)\right]
\end{aligned}
$$

for all $t \geq t_{0}$ and $(x, y) \in \bar{\Omega}$.

Proof. Step 1: Choice of $t_{0}>0$. Since $u$ and $U$ solve the same equation 1.1p with $p^{-}(x, y) \leq u(t, x, y), U(t, x, y) \leq p^{+}(x, y)$ for all $(t, x, y) \in[0,+\infty) \times \bar{\Omega}$, we have

$$
|u(t, x, y)-U(t, x, y)| \leq e^{M t}\left|u_{0}(x, y)-U(0, x, y)\right|
$$

for all $(t, x, y) \in[0,+\infty) \times \bar{\Omega}$, where $M \in[0,+\infty)$ is defined in 3.19]. In particular, it follows from 1.20 that, for each $t>0$,

$u(t, x, y)-p^{-}(x, y)=U(t, x, y)-p^{-}(x, y)+o\left(U(0, x, y)-p^{-}(x, y)\right)$ as $x \cdot e \rightarrow+\infty$. 
Since both $U$ and $p^{-}$satisfy 11.1 and $U>p^{-}$in $\mathbb{R} \times \bar{\Omega}$, it follows from the Harnack inequality that, for each $t>0$, there is a constant $C_{t}>0$ such that

$$
0<U(0, x, y)-p^{-}(x, y) \leq C_{t}\left(U(t, x, y)-p^{-}(x, y)\right) \quad \text { for all }(x, y) \in \bar{\Omega} .
$$

As a consequence,

$$
\forall t>0, \quad u(t, x, y)-p^{-}(x, y) \sim U(t, x, y)-p^{-}(x, y) \quad \text { as } x \cdot e \rightarrow+\infty .
$$

It also follows from (1.19) and 3.22 that one can choose $t_{0}>0$ small enough so that

$$
\liminf _{\varsigma \rightarrow-\infty} \inf _{(x, y) \in \bar{\Omega}, x \cdot e \leq \varsigma} \frac{u\left(t_{0}, x, y\right)-p^{+}(x, y)}{\psi^{+}(x, y)}>-\frac{\varepsilon_{0} e^{-\omega t_{0}}}{m^{+}}=-\varepsilon_{1} e^{-\omega t_{0}},
$$

because of 3.18 . Since $0<2 \varepsilon_{1} \leq \rho_{1}^{+} / 2 \leq \rho^{+}$, it follows from 3.9 and 3.24 that

$$
\begin{aligned}
\sup _{(s, x, y) \in \mathbb{R} \times \bar{\Omega}} \frac{\phi(s, x, y)-2 \varepsilon_{1} g\left(s+s_{0}, x, y\right) e^{-\omega t_{0}}-p^{+}(x, y)}{\psi^{+}(x, y)} \\
<\liminf _{\zeta \rightarrow-\infty} \inf _{(x, y) \in \bar{\Omega}, x \cdot e \leq \varsigma} \frac{u\left(t_{0}, x, y\right)-p^{+}(x, y)}{\psi^{+}(x, y)} .
\end{aligned}
$$

Step 2: Choice of $\sigma_{0} \geq \underline{\sigma}$. We now claim that

$$
\begin{aligned}
\max \left[\phi\left(s_{\sigma}\left(t_{0}, x\right), x, y\right)-2 \varepsilon_{1} g\left(s_{\sigma}\left(t_{0}, x\right)+s_{0}, x, y\right) e^{-\omega t_{0}}, p^{-}(x, y)\right] & \\
& \leq u\left(t_{0}, x, y\right) \text { in } \bar{\Omega}
\end{aligned}
$$

for all $\sigma>0$ large enough. Assume not. Then there exist sequences $\left(x_{n}, y_{n}\right)_{n \in \mathbb{N}}$ in $\bar{\Omega}$ and $\left(\sigma_{n}\right)_{n \in \mathbb{N}}$ such that $\lim _{n \rightarrow+\infty} \sigma_{n}=+\infty$ and

$$
\begin{array}{r}
\max \left[\phi\left(s_{\sigma_{n}}\left(t_{0}, x_{n}\right), x_{n}, y_{n}\right)-2 \varepsilon_{1} g\left(s_{\sigma_{n}}\left(t_{0}, x_{n}\right)+s_{0}, x_{n}, y_{n}\right) e^{-\omega t_{0}}, p^{-}\left(x_{n}, y_{n}\right)\right] \\
>u\left(t_{0}, x_{n}, y_{n}\right)
\end{array}
$$

for all $n \in \mathbb{N}$. Since $u \geq p^{-}$, one gets

$$
\phi\left(s_{\sigma_{n}}\left(t_{0}, x_{n}\right), x_{n}, y_{n}\right)-2 \varepsilon_{1} g\left(s_{\sigma_{n}}\left(t_{0}, x_{n}\right)+s_{0}, x_{n}, y_{n}\right) e^{-\omega t_{0}}>u\left(t_{0}, x_{n}, y_{n}\right)
$$

for all $n \in \mathbb{N}$. Up to taking a subsequence, two cases may occur: either the sequence $\left(s_{\sigma_{n}}\left(t_{0}, x_{n}\right)\right)_{n \in \mathbb{N}}$ is bounded from above, or $\lim _{n \rightarrow+\infty} s_{\sigma_{n}}\left(t_{0}, x_{n}\right)=+\infty$. If the sequence is bounded from above, then $x_{n} \cdot e \rightarrow-\infty$ as $n \rightarrow+\infty$. We have

$$
\begin{gathered}
\frac{\phi\left(s_{\sigma_{n}}\left(t_{0}, x_{n}\right), x_{n}, y_{n}\right)-2 \varepsilon_{1} g\left(s_{\sigma_{n}}\left(t_{0}, x_{n}\right)+s_{0}, x_{n}, y_{n}\right) e^{-\omega t_{0}}-p^{+}\left(x_{n}, y_{n}\right)}{\psi^{+}\left(x_{n}, y_{n}\right)}>\frac{u\left(t_{0}, x_{n}, y_{n}\right)-p^{+}\left(x_{n}, y_{n}\right)}{\psi^{+}\left(x_{n}, y_{n}\right)} .
\end{gathered}
$$


But the limsup of the left-hand side as $n \rightarrow+\infty$ is less than the liminf of the right-hand side, because of 3.25 and $\lim _{n \rightarrow+\infty} x_{n} \cdot e=-\infty$. This case is thus ruled out.

Consequently, $s_{\sigma_{n}}\left(t_{0}, x_{n}\right) \rightarrow+\infty$ as $n \rightarrow+\infty$. As $\phi(+\infty, \cdot, \cdot)=p^{-}$and $u \geq p^{-}$, it follows from 3.27 that

$$
u\left(t_{0}, x_{n}, y_{n}\right)-p^{-}\left(x_{n}, y_{n}\right) \rightarrow 0 \quad \text { as } n \rightarrow+\infty .
$$

From (3.24) and $0<\varepsilon_{1} e^{-\omega t_{0}}<\varepsilon_{1} \leq \rho^{+} / 2<\rho^{+}=\min _{\bar{\Omega}}\left[\left(p^{+}-p^{-}\right) / \psi^{+}\right]$, it follows, as in the proof of Lemma 3.1, that the sequence $\left(x_{n} \cdot e\right)_{n \in \mathbb{N}}$ is bounded from below. Up to taking another subsequence, two subcases may occur: either the sequence $\left(x_{n} \cdot e\right)_{n \in \mathbb{N}}$ is bounded, or it converges to $+\infty$ as $n \rightarrow+\infty$. Write $x_{n}=x_{n}^{\prime}+x_{n}^{\prime \prime}$ where $x_{n}^{\prime} \in$ $L_{1} \mathbb{Z} \times \cdots \times L_{d} \mathbb{Z}$ and $\left(x_{n}^{\prime \prime}, y_{n}\right) \in C$ for all $n \in \mathbb{N}$. Up to taking a subsequence, one can assume that $\left(x_{n}^{\prime \prime}, y_{n}\right) \rightarrow\left(x_{\infty}, y_{\infty}\right) \in C$ as $n \rightarrow+\infty$. Set

$$
u_{n}(t, x, y)=u_{n}\left(t, x+x_{n}^{\prime}, y\right) .
$$

By periodicity of coefficients of (1.1), the functions $u_{n}$ solve (1.1) for $t>0$. Furthermore, $p^{-}(x, y) \leq u_{n}(t, x, y) \leq p^{+}(x, y)$ for all $(t, x, y) \in[0,+\infty) \times \bar{\Omega}$ and $n \in \mathbb{N}$. From standard parabolic estimates, the functions $u_{n}$ converge locally uniformly in $(0,+\infty) \times \bar{\Omega}$, up to taking a subsequence, to a solution $u_{\infty}$ of (1.1) such that

$$
p^{-}(x, y) \leq u_{\infty}(t, x, y) \leq p^{+}(x, y) \quad \text { for all }(t, x, y) \in(0,+\infty) \times \bar{\Omega} .
$$

Moreover, $u_{\infty}\left(t_{0}, x_{\infty}, y_{\infty}\right)=p^{-}\left(x_{\infty}, y_{\infty}\right)$ from 3.28). It follows from the strong maximum principle that $u_{\infty}(t, x, y)=p^{-}(x, y)$ for all $(t, x, y) \in\left(0, t_{0}\right] \times \bar{\Omega}$ (and so in $(0,+\infty) \times \bar{\Omega})$. If the sequence $\left(x_{n} \cdot e\right)_{n \in \mathbb{N}}$ is bounded, so is $\left(x_{n}^{\prime} \cdot e\right)_{n \in \mathbb{N}}$, hence the function $u_{\infty}$ still satisfies (3.24). This leads to a contradiction as above. Therefore, $x_{n} \cdot e \rightarrow+\infty$ as $n \rightarrow+\infty$. By (3.27),

$$
\frac{\phi\left(s_{\sigma_{n}}\left(t_{0}, x_{n}\right), x_{n}, y_{n}\right)-p^{-}\left(x_{n}, y_{n}\right)}{U\left(t_{0}, x_{n}, y_{n}\right)-p^{-}\left(x_{n}, y_{n}\right)}>\frac{u\left(t_{0}, x_{n}, y_{n}\right)-p^{-}\left(x_{n}, y_{n}\right)}{U\left(t_{0}, x_{n}, y_{n}\right)-p^{-}\left(x_{n}, y_{n}\right)} .
$$

Because of 3.23, the right-hand side converges to 1 as $n \rightarrow+\infty$. On the other hand, the left-hand side is equal to

$$
\begin{aligned}
& \frac{\phi\left(s_{\sigma_{n}}\left(t_{0}, x_{n}\right), x_{n}, y_{n}\right)-p^{-}\left(x_{n}, y_{n}\right)}{U\left(t_{0}, x_{n}, y_{n}\right)-p^{-}\left(x_{n}, y_{n}\right)} \\
& =\frac{\phi\left(x_{n} \cdot e-c t_{0}+\sigma_{n}-\sigma_{n} e^{-\omega t_{0}}, x_{n}, y_{n}\right)-p^{-}\left(x_{n}, y_{n}\right)}{\phi\left(x_{n} \cdot e-c t_{0}, x_{n}, y_{n}\right)-p^{-}\left(x_{n}, y_{n}\right)} .
\end{aligned}
$$

But property (2.2), together with $\lim _{n \rightarrow+\infty} x_{n} \cdot e=\lim _{n \rightarrow+\infty} \sigma_{n}=+\infty$, implies that the above quantity converges to 0 as $n \rightarrow+\infty$. This leads to a contradiction. Eventually, the claim 3.26 is proved.

Next, we claim that

$$
u\left(t_{0}, x, y\right) \leq \min \left\{\phi\left(s_{-\sigma}\left(t_{0}, x\right), x, y\right)+g\left(s_{-\sigma}\left(t_{0}, x\right), x, y\right) e^{-\omega t_{0}}, p^{+}(x, y)\right\}
$$


in $\bar{\Omega}$ for all $\sigma>0$ large enough. Assume not. Since $u \leq p^{+}$, there exist sequences $\left(x_{n}, y_{n}\right)_{n \in \mathbb{N}}$ in $\bar{\Omega}$ and $\left(\sigma_{n}\right)_{n \in \mathbb{N}}$ such that $\lim _{n \rightarrow+\infty} \sigma_{n}=+\infty$ and

$$
\phi\left(s_{-\sigma_{n}}\left(t_{0}, x_{n}\right), x_{n}, y_{n}\right)+g\left(s_{-\sigma_{n}}\left(t_{0}, x_{n}\right), x_{n}, y_{n}\right) e^{-\omega t_{0}}<u\left(t_{0}, x_{n}, y_{n}\right)
$$

for all $n \in \mathbb{N}$. If $s_{-\sigma_{n}}\left(t_{0}, x_{n}\right) \rightarrow-\infty$ as $n \rightarrow+\infty$ up to taking a subsequence, then $\phi\left(s_{-\sigma_{n}}\left(t_{0}, x_{n}\right), x_{n}, y_{n}\right)-p^{+}\left(x_{n}, y_{n}\right) \rightarrow 0$, while $u\left(t_{0}, x_{n}, y_{n}\right) \leq p^{+}\left(x_{n}, y_{n}\right)$ and $\liminf _{n \rightarrow+\infty} g\left(s_{-\sigma_{n}}\left(t_{0}, x_{n}\right), x_{n}, y_{n}\right) e^{-\omega t_{0}} \geq m^{+} e^{-\omega t_{0}}>0$, where $m^{+}=\min _{\bar{\Omega}} \psi^{+}>0$. This gives a contradiction. Thus, the sequence $\left(s_{-\sigma_{n}}\left(t_{0}, x_{n}\right)\right)_{n \in \mathbb{N}}$ is bounded from below, whence $x_{n} \cdot e \rightarrow+\infty$ as $n \rightarrow+\infty$. In particular, $u\left(t_{0}, x_{n}, y_{n}\right)-p^{-}\left(x_{n}, y_{n}\right) \rightarrow 0$ as $n \rightarrow$ $+\infty$ from 3.23. Since $\phi \geq p^{-}$and $g \geq 0$, one finds that $g\left(s_{-\sigma_{n}}\left(t_{0}, x_{n}\right), x_{n}, y_{n}\right) \rightarrow 0$ as $n \rightarrow+\infty$, whence $s_{-\sigma_{n}}\left(t_{0}, x_{n}\right) \rightarrow+\infty$ owing to the definition of $g$. Moreover,

$$
\frac{\phi\left(s_{-\sigma_{n}}\left(t_{0}, x_{n}\right), x_{n}, y_{n}\right)-p^{-}\left(x_{n}, y_{n}\right)}{U\left(t_{0}, x_{n}, y_{n}\right)-p^{-}\left(x_{n}, y_{n}\right)}<\frac{u\left(t_{0}, x_{n}, y_{n}\right)-p^{-}\left(x_{n}, y_{n}\right)}{U\left(t_{0}, x_{n}, y_{n}\right)-p^{-}\left(x_{n}, y_{n}\right)}
$$

and the right-hand side converges to 1 as $n \rightarrow+\infty$ from 3.23 . Since

$$
\lim _{n \rightarrow+\infty} s_{-\sigma_{n}}\left(t_{0}, x_{n}\right)=\lim _{n \rightarrow+\infty} x_{n} \cdot e=\lim _{n \rightarrow+\infty}\left(x_{n} \cdot e-s_{-\sigma_{n}}\left(t_{0}, x_{n}\right)\right)=+\infty
$$

one concludes from (2.2) that the left-hand side of (3.30) converges to $+\infty$ as $n \rightarrow+\infty$, which is a contradiction. Hence, the claim (3.29) is proved.

In the rest of the proof, we fix a real number $\sigma_{0}$ large enough so that 3.26 and 3.29) hold for $\sigma=\sigma_{0}$, and $\sigma_{0} \geq \underline{\sigma} \geq 0$, where $\underline{\sigma} \geq 0$ has been given in 3.20p.

Step 3: The lower and upper bounds in (3.21) are sub- and super-solutions of (1.1). Define

$$
\mathcal{L} w=w_{t}-\nabla \cdot(A(x, y) \nabla w)+q(x, y) \cdot \nabla w-f(x, y, w)
$$

and

$$
\begin{aligned}
& \underline{u}(t, x, y)=\phi\left(s_{\sigma_{0}}(t, x), x, y\right)-2 \varepsilon_{1} g\left(s_{\sigma_{0}}(t, x)+s_{0}, x, y\right) e^{-\omega t}, \\
& \bar{u}(t, x, y)=\phi\left(s_{-\sigma_{0}}(t, x), x, y\right)+g\left(s_{-\sigma_{0}}(t, x), x, y\right) e^{-\omega t},
\end{aligned}
$$

for all $(t, x, y) \in\left[t_{0},+\infty\right) \times \bar{\Omega}$.

Since $v A \nabla U(t, \cdot, \cdot)=v A \nabla \psi^{+}=v A \nabla \psi-\lambda(\nu A e) \psi=v A \nabla \theta+v A e=0$ on $\partial \Omega$, it is immediate from the definitions of $g$ and $s_{ \pm \sigma_{0}}(t, x)$ that

$$
v A(x, y) \nabla \underline{u}(t, x, y)=v A(x, y) \nabla \bar{u}(t, x, y)=0
$$

for all $(t, x, y) \in\left[t_{0},+\infty\right) \times \partial \Omega$.

Recall now that $p^{-} \leq u \leq p^{+}$solves 1.1 , and that the inequalities 3.21 are satisfied at time $t_{0}$. In order to prove 3.21 for all $(t, x, y) \in\left[t_{0},+\infty\right) \times \bar{\Omega}$, it is thus enough to prove, from the maximum principle, that

$$
\mathcal{L} \underline{u} \leq 0 \text { in } \Omega_{-} \quad \text { and } \quad \mathcal{L} \bar{u} \geq 0 \text { in } \Omega_{+},
$$


where

$$
\begin{aligned}
& \Omega_{-}=\left\{(t, x, y) \in\left[t_{0},+\infty\right) \times \bar{\Omega}: \underline{u}(t, x, y)>p^{-}(x, y)\right\}, \\
& \Omega_{+}=\left\{(t, x, y) \in\left[t_{0},+\infty\right) \times \bar{\Omega}: \bar{u}(t, x, y)<p^{+}(x, y)\right\}
\end{aligned}
$$

Let us first deal with the function $\underline{u}$. By using equations (1.1), (1.17), 3.3) and $L_{\lambda} \psi=$ $k(\lambda) \psi$ in $\bar{\Omega}$, a lengthy but straightforward calculation leads to, for all $(t, x, y) \in \Omega_{-}$,

$$
\begin{aligned}
\mathcal{L} \underline{u}(t, x, y)= & f\left(x, y, \phi\left(s_{\sigma_{0}}(t, x), x, y\right)\right)-f(x, y, \underline{u}(t, x, y)) \\
& +\sigma_{0} \omega \phi_{s}\left(s_{\sigma_{0}}(t, x), x, y\right) e^{-\omega t} \\
& -2 \varepsilon_{1} B\left(s_{\sigma_{0}}(t, x)+s_{0}, x, y\right) e^{-\omega t}-2 \varepsilon_{1} \sigma_{0} \omega C\left(s_{\sigma_{0}}(t, x)+s_{0}, x, y\right) e^{-2 \omega t},
\end{aligned}
$$

where the functions $B$ and $C$ have been defined in $(3.10)$.

If $(t, x, y) \in \Omega_{-}$and $s_{\sigma_{0}}(t, x) \geq s^{+}$, where $s^{+}$is given by 3.13 , then

$$
\begin{aligned}
f\left(x, y, \phi\left(s_{\sigma_{0}}(t, x), x, y\right)\right)-f(x, y, \underline{u}(t, x, y)) & \\
& \leq 2 \varepsilon_{1}\left(\zeta^{-}(x, y)+\omega\right) g\left(s_{\sigma_{0}}(t, x)+s_{0}, x, y\right) e^{-\omega t}
\end{aligned}
$$

from (3.12) and (3.13), whence

$$
\begin{aligned}
\mathcal{L} \underline{u}(t, x, y) \leq & 2 \varepsilon_{1}\left[\left(\zeta^{-}(x, y)+\omega\right) g\left(s_{\sigma_{0}}(t, x)+s_{0}, x, y\right)-B\left(s_{\sigma_{0}}(t, x)+s_{0}, x, y\right)\right] e^{-\omega t} \\
& +\sigma_{0} \omega\left[\phi_{s}\left(s_{\sigma_{0}}(t, x), x, y\right)-2 \varepsilon_{1} C\left(s_{\sigma_{0}}(t, x)+s_{0}, x, y\right) e^{-\omega t}\right] e^{-\omega t} \\
\leq & 0
\end{aligned}
$$

because of 3.13 and $0<2 \varepsilon_{1} e^{-\omega t} \leq \rho^{+}$.

If $(t, x, y) \in \Omega_{-}$and $s_{\sigma_{0}}(t, x) \leq s^{-}$, where $s^{-}$is given by 3.15 , then $g\left(s_{\sigma_{0}}(t, x)+\right.$ $\left.s_{0}, x, y\right)=\psi^{+}(x, y)$ (because $\left.s_{0} \leq 0\right)$ and

$$
\begin{aligned}
p^{+}(x, y) & >\phi\left(s_{\sigma_{0}}(t, x), x, y\right) \geq \underline{u}(t, x, y) \\
& \geq p^{+}(x, y)-\frac{\rho_{1}^{+}}{2} \psi^{+}(x, y)-2 \varepsilon_{1} \psi^{+}(x, y) e^{-\omega t} \geq p^{+}(x, y)-\rho_{1}^{+} \psi^{+}(x, y)
\end{aligned}
$$

because $\varepsilon_{1} \leq \rho_{1}^{+} / 4$ from 3.17). Thus,

$$
f\left(x, y, \phi\left(s_{\sigma_{0}}(t, x), x, y\right)\right)-f(x, y, \underline{u}(t, x, y)) \leq 2 \varepsilon_{1}\left(\zeta^{+}(x, y)+\omega\right) \psi^{+}(x, y) e^{-\omega t}
$$

from 3.14. Since $\phi_{s}<0$ and since the last two properties in 3.15) also hold with $s+s_{0}$ instead of $s$ (because $s_{0} \leq 0$ ), it follows that

$$
\begin{aligned}
\mathcal{L} \underline{u}(t, x, y) \leq & 2 \varepsilon_{1}\left(\zeta^{+}(x, y)+\omega\right) \psi^{+}(x, y) e^{-\omega t}-2 \varepsilon_{1}\left(\zeta^{+}(x, y)\right. \\
& \left.+\mu^{+}-\omega\right) \psi^{+}(x, y) e^{-\omega t} \\
= & 2 \varepsilon_{1}\left(2 \omega-\mu^{+}\right) \psi^{+}(x, y) e^{-\omega t} \leq 0
\end{aligned}
$$

from 3.2 and 3.3 . 
If $(t, x, y) \in \Omega_{-}$and $s^{-} \leq s_{\sigma_{0}}(t, x) \leq s^{+}$, it follows from the definitions of $\delta, \varepsilon_{1}, M$ and $\sigma$ in 3.16, 3.17, 3.19) and 3.20, together with the inequality $\sigma_{0} \geq \underline{\sigma}$, that

$$
\begin{aligned}
\mathcal{L} \underline{u}(t, x, y) & \leq 2 \varepsilon_{1} M\|g\|_{\infty} e^{-\omega t}+2 \varepsilon_{1}\|B\|_{\infty} e^{-\omega t}-\sigma_{0} \omega \delta e^{-\omega t}+2 \varepsilon_{1} \sigma_{0} \omega\|C\|_{\infty} e^{-2 \omega t} \\
& \leq \frac{\delta\left(M\|g\|_{\infty}+\|B\|_{\infty}\right) e^{-\omega t}}{2\|C\|_{\infty}}-\frac{\sigma_{0} \omega \delta e^{-\omega t}}{2} \leq 0
\end{aligned}
$$

As a consequence, $\underline{u}$ is a sub-solution of $\sqrt{1.1}$ in $\Omega_{-}$, and $\underline{u}\left(t_{0}, \cdot, \cdot\right) \leq u\left(t_{0}, \cdot, \cdot\right)$ in $\bar{\Omega}$. Thus, $\underline{u}(t, x, y) \leq u(t, \bar{x}, y)$ for all $(t, x, y) \in\left[t_{0},+\infty\right) \times \bar{\Omega}$ from the parabolic maximum principle.

Let us now check that $\mathcal{L} \bar{u}(t, x, y) \geq 0$ for all $(t, x, y) \in \Omega^{+}$. Just as for $\underline{u}$, it is straightforward to check that

$$
\begin{aligned}
\mathcal{L} \bar{u}(t, x, y)= & f\left(x, y, \phi\left(s_{-\sigma_{0}}(t, x), x, y\right)\right)-f(x, y, \bar{u}(t, x, y)) \\
& -\sigma_{0} \omega \phi_{S}\left(s_{-\sigma_{0}}(t, x), x, y\right) e^{-\omega t}+B\left(s_{-\sigma_{0}}(t, x), x, y\right) e^{-\omega t} \\
& -\sigma_{0} \omega C\left(s_{\sigma_{0}}(t, x), x, y\right) e^{-2 \omega t} \\
\geq & f\left(x, y, \phi\left(s_{-\sigma_{0}}(t, x), x, y\right)\right)-f(x, y, \bar{u}(t, x, y)) \\
& -\sigma_{0} \omega \phi_{S}\left(s_{-\sigma_{0}}(t, x), x, y\right) e^{-\omega t}+B\left(s_{-\sigma_{0}}(t, x), x, y\right) e^{-\omega t}
\end{aligned}
$$

from 3.11$)$.

If $(t, x, y) \in \Omega_{+}$and $s_{-\sigma_{0}}(t, x) \geq s^{+}$, where $s^{+}$is given by 3.13 , then

$$
p^{-}(x, y)<\phi\left(s_{-\sigma_{0}}(t, x), x, y\right) \leq \bar{u}(t, x, y) \leq p^{-}(x, y)+\rho^{-}
$$

(notice indeed that the first four properties in 3.13 hold without $s_{0}$, since $s_{0} \leq 0$ ). As $\phi_{s}<0$, it then follows from (3.12) and (3.13) that $\mathcal{L} \bar{u}(t, x, y) \geq 0$.

If $(t, x, y) \in \Omega_{+}$and $s_{-\sigma_{0}}(t, x) \leq s^{-}$, where $s^{-}$is given by 3.15$)$, then

$$
p^{+}(x, y)-\rho_{1}^{+} \psi^{+}(x, y) \leq \phi\left(s_{-\sigma_{0}}(t, x), x, y\right) \leq \bar{u}(t, x, y)<p^{+}(x, y),
$$

whence

$$
\begin{aligned}
\mathcal{L} \bar{u}(t, x, y) & \geq-\left(\zeta^{+}(x, y)+\omega\right) \psi^{+}(x, y) e^{-\omega t}+\left(\zeta^{+}(x, y)+\mu^{+}-\omega\right) \psi^{+}(x, y) e^{-\omega t} \\
& =\left(\mu^{+}-2 \omega\right) \psi^{+}(x, y) e^{-\omega t} \geq 0
\end{aligned}
$$

from (3.2), 3.3), 3.14) and 3.15).

If $(t, x, y) \in \Omega_{+}$and $s^{-} \leq s_{-\sigma_{0}}(t, x) \leq s^{+}$, it follows from $3.16, \sqrt{3.19}, \sqrt{3.20}$ and the inequality $\sigma_{0} \geq \underline{\sigma}$ that

$$
\begin{aligned}
\mathcal{L} \bar{u}(t, x, y) & \geq-M\|g\|_{\infty} e^{-\omega t}+\sigma_{0} \omega \delta e^{-\omega t}-\|B\|_{\infty} e^{-\omega t} \\
& \geq\left(\underline{\sigma} \omega \delta-M\|g\|_{\infty}-\|B\|_{\infty}\right) e^{-\omega t} \geq 0 .
\end{aligned}
$$

Consequently, the parabolic maximum principle yields $u(t, x, y) \leq \bar{u}(t, x, y)$ for all $(t, x, y) \in\left[t_{0},+\infty\right) \times \bar{\Omega}$, and the proof of Proposition 3.2 is complete.

The following proposition states that the solution $u$ stays close to the front $\phi$ when $x \cdot e-c t$ is very positive. 
Proposition 3.3. Under all assumptions of Theorem 1.3 and under the above notations, there exists $\bar{\sigma} \in \mathbb{R}$ such that, for each $\eta>0$, there is $D_{\eta}>0$ such that, for all $(t, x, y) \in$ $[0,+\infty) \times \bar{\Omega}$,

$$
\phi(x \cdot e-c t+\eta, x, y)-D_{\eta} \psi(x, y) e^{-\lambda(x \cdot e-c t)} \leq u(t, x, y)
$$

and

$$
[x \cdot e-c t \geq \bar{\sigma}] \Rightarrow\left[u(t, x, y) \leq \phi(x \cdot e-c t-\eta, x, y)+D_{\eta} \psi(x, y) e^{-\lambda(x \cdot e-c t)}\right] .
$$

Proof. Let $t_{0}>0$ and $\sigma_{0} \geq \underline{\sigma} \geq 0$ be as in Proposition 3.2. Recall that $\phi(+\infty, \cdot, \cdot)$ $=p^{-}$uniformly in $\bar{\Omega}$. It follows from $\sqrt{3.21}$ and the definition of $g$ and $\chi$ that there exists $\sigma \in \mathbb{R}$ such that, for all $(t, x, y) \in\left[t_{0},+\infty\right) \times \bar{\Omega}$ with $x \cdot e-c t \geq \sigma$,

$$
\begin{aligned}
u(t, x, y) & \leq \phi\left(x \cdot e-c t-\sigma_{0}+\sigma_{0} e^{-\omega t}, x, y\right)+\psi(x, y) e^{-\lambda\left(x \cdot e-c t-\sigma_{0}+\sigma_{0} e^{-\omega t}\right)} e^{-\omega t} \\
& \leq p^{-}(x, y)+\rho^{-},
\end{aligned}
$$

where $\rho^{-}>0$ is as in 3.12 . On the other hand, for all $(t, x, y) \in[0,+\infty) \times \bar{\Omega}$,

$$
u(t, x, y) \leq \phi(x \cdot e-c t, x, y)+e^{M t}\left|u_{0}(x, y)-U(0, x, y)\right|
$$

from 3.22$)$, and $u_{0}(x, y)-U(0, x, y) \rightarrow 0$ uniformly as $x \cdot e \rightarrow+\infty$ from assumption 1.20 . Therefore, there exists $\bar{\sigma} \geq \sigma$ such that, for all $(t, x, y) \in\left[0, t_{0}\right] \times \bar{\Omega}$ with $x \cdot e-$ $c t \geq \bar{\sigma}$, we have $u(t, x, y) \leq p^{-}(x, y)+\rho^{-}$. To sum up, for all $(t, x, y) \in[0,+\infty) \times \bar{\Omega}$,

$$
[x \cdot e-c t \geq \bar{\sigma}] \Rightarrow\left[u(t, x, y) \leq p^{-}(x, y)+\rho^{-}\right] .
$$

Let $\eta>0$ be any positive number. We claim that

$$
\phi(x \cdot e+\eta, x, y)-D \psi(x, y) e^{-\lambda x \cdot e} \leq u_{0}(x, y) \quad \text { in } \bar{\Omega}
$$

for $D$ large enough. Assume not. Then there exist sequences $\left(x_{n}, y_{n}\right)_{n \in \mathbb{N}}$ in $\bar{\Omega}$ and $\left(D_{n}\right)_{n \in \mathbb{N}}$ in $[0,+\infty)$ such that $\lim _{n \rightarrow+\infty} D_{n}=+\infty$ and

$$
\phi\left(x_{n} \cdot e+\eta, x_{n}, y_{n}\right)-D_{n} \psi\left(x_{n}, y_{n}\right) e^{-\lambda x_{n} \cdot e}>u_{0}\left(x_{n}, y_{n}\right)
$$

for all $n \in \mathbb{N}$. Since $\phi$ and $u_{0}$ are bounded and $\min _{\bar{\Omega}} \psi>0$, it follows that $\lim _{n \rightarrow+\infty} x_{n} \cdot e$ $=+\infty$. For all $n \in \mathbb{N}$,

$$
\frac{\phi\left(x_{n} \cdot e+\eta, x_{n}, y_{n}\right)-p^{-}\left(x_{n}, y_{n}\right)}{\phi\left(x_{n} \cdot e, x_{n}, y_{n}\right)-p^{-}\left(x_{n}, y_{n}\right)}>\frac{u_{0}\left(x_{n}, y_{n}\right)-p^{-}\left(x_{n}, y_{n}\right)}{\phi\left(x_{n} \cdot e, x_{n}, y_{n}\right)-p^{-}\left(x_{n}, y_{n}\right)} .
$$

The right-hand side converges to 1 as $n \rightarrow+\infty$, from assumption $(1.20)$, while the limsup of the left-hand side is not larger than $e^{-\lambda_{m, \phi} \eta}<1$, from 2.2. One has thus reached a contradiction. Hence, 3.32 holds for $D$ large enough.

Similarly, it is easy to check that

$$
u_{0}(x, y) \leq \phi(x \cdot e-\eta, x, y)+D \psi(x, y) e^{-\lambda x \cdot e} \quad \text { in } \bar{\Omega}
$$

for $D$ large enough. 
We choose $D_{\eta}>0$ such that 3.32 and 3.33 hold for $D=D_{\eta}$, and

$$
D_{\eta} \geq \max \left(e^{\lambda s^{+}} \max _{\bar{\Omega}} \frac{p^{+}-p^{-}}{\psi}, \rho^{-} e^{\lambda \bar{\sigma}} \max _{\bar{\Omega}} \frac{1}{\psi}\right)
$$

where $s^{+}$and $\bar{\sigma}$ have been given in 3.13 and 3.31 .

Set

$$
\underline{u}_{\eta}(t, x, y)=\phi(x \cdot e-c t+\eta, x, y)-D_{\eta} \psi(x, y) e^{-\lambda(x \cdot e-c t)}
$$

for all $(t, x, y) \in[0,+\infty) \times \bar{\Omega}$. Notice that $\underline{u}_{\eta}(0, x, y) \leq u(0, x, y)$ in $\bar{\Omega}, u \geq p^{-}$and $v A(x, y) \nabla \underline{u}_{\eta}(t, x, y)=0$ for all $(t, x, y) \in[0,+\infty) \times \partial \Omega$. In order to prove that $\underline{u}_{\eta} \leq u$ in $[0,+\infty) \times \bar{\Omega}$, it is then sufficient to check that $\mathcal{L}_{\eta}(t, x, y) \leq 0$ for all $(t, x, y) \in$ $[0,+\infty) \times \bar{\Omega}$ such that $\underline{u}_{\eta}(t, x, y)>p^{-}(x, y)$. From $1.1,3.3$ and $L_{\lambda} \psi=k(\lambda) \psi$ in $\bar{\Omega}$,

$$
\begin{aligned}
\mathcal{L}_{\underline{u}_{\eta}}(t, x, y)= & f(x, y, \phi(x \cdot e-c t+\eta, x, y))-f\left(x, y, \underline{u}_{\eta}(t, x, y)\right) \\
& -\left(2 \omega+\zeta^{-}(x, y)\right) D_{\eta} \psi(x, y) e^{-\lambda(x \cdot e-c t)}
\end{aligned}
$$

for all $(t, x, y) \in[0,+\infty) \times \bar{\Omega}$. When $\underline{u}_{\eta}(t, x, y)>p^{-}(x, y)$, then

$$
D_{\eta} \psi(x, y) e^{-\lambda(x \cdot e-c t)}<\phi(x \cdot e-c t+\eta, x, y)-p^{-}(x, y)<p^{+}(x, y)-p^{-}(x, y),
$$

whence $D_{\eta} e^{-\lambda(x \cdot e-c t)} \leq \max _{\bar{\Omega}}\left[\left(p^{+}-p^{-}\right) / \psi\right]$. Because of 3.34$)$, it follows that $x \cdot e-c t$ $\geq s^{+}$, and so

$$
\phi(x \cdot e-c t+\eta, x, y)<\phi\left(s^{+}, x, y\right) \leq p^{-}(x, y)+\rho^{-} / 2
$$

from 3.13. In particular, when $\underline{u}_{\eta}(t, x, y)>p^{-}(x, y)$, then

$$
p^{-}(x, y)<\underline{u}_{\eta}(t, x, y)<\phi(x \cdot e-c t+\eta, x, y)<p^{-}(x, y)+\rho^{-},
$$

whence

$$
\begin{aligned}
f(x, y, \phi(x \cdot e-c t+\eta, x, y))-f(x, y & \left.\underline{u}_{\eta}(t, x, y)\right) \\
& \leq\left(\zeta^{-}(x, y)+\omega\right) D_{\eta} \psi(x, y) e^{-\lambda(x \cdot e-c t)}
\end{aligned}
$$

from 3.12 . It follows that $\mathcal{L}_{\underline{\eta}}(t, x, y) \leq-\omega D_{\eta} \psi(x, y) e^{-\lambda(x \cdot e-c t)}<0$ for all $(t, x, y)$ $\in[0,+\infty) \times \bar{\Omega}$ such that $\underline{u}_{\eta}(t, x, y)>p^{-}(x, y)$. The maximum principle then yields $\underline{u}_{\eta}(t, x, y) \leq u(t, x, y)$ for all $(t, x, y) \in[0,+\infty) \times \bar{\Omega}$.

Now, set

$$
\bar{u}_{\eta}(t, x, y)=\phi(x \cdot e-c t-\eta, x, y)+D_{\eta} \psi(x, y) e^{-\lambda(x \cdot e-c t)}
$$

for all $(t, x, y) \in[0,+\infty) \times \bar{\Omega}$. Notice that $u(0, x, y) \leq \bar{u}_{\eta}(0, x, y)$ in $\bar{\Omega}$, that $\nu A(x, y) \nabla \bar{u}_{\eta}(t, x, y)=0$ for all $(t, x, y) \in[0,+\infty) \times \partial \Omega$, that

$$
[x \cdot e-c t \geq \bar{\sigma}] \Rightarrow\left[u(t, x, y) \leq p^{-}(x, y)+\rho^{-}\right]
$$


from 3.31 , and that

$$
[x \cdot e-c t=\bar{\sigma}] \Rightarrow\left[\bar{u}_{\eta}(t, x, y)>p^{-}(x, y)+\rho^{-}\right]
$$

from 3.34 and $\phi>p^{-}$. In order to prove that $u \leq \bar{u}_{\eta}$ when $x \cdot e-c t \geq \bar{\sigma}$, it is thus sufficient to check that $\mathcal{L} \bar{u}_{\eta}(t, x, y) \geq 0$ for all $(t, x, y) \in[0,+\infty) \times \bar{\Omega}$ such that $\bar{u}_{\eta}(t, x, y) \leq p^{-}(x, y)+\rho^{-}$. For all such $(t, x, y)$,

$$
\begin{aligned}
\mathcal{L} \bar{u}_{\eta}(t, x, y)= & f(x, y, \phi(x \cdot e-c t-\eta, x, y))-f\left(x, y, \bar{u}_{\eta}(t, x, y)\right) \\
& +\left(2 \omega+\zeta^{-}(x, y)\right) D_{\eta} \psi(x, y) e^{-\lambda(x \cdot e-c t)}
\end{aligned}
$$

and $p^{-}(x, y)<\phi(x \cdot e-c t-\eta, x, y)<\bar{u}_{\eta}(t, x, y) \leq p^{-}(x, y)+\rho^{-}$, whence

$$
\begin{aligned}
\mathcal{L} \bar{u}_{\eta}(t, x, y) \geq & -\left(\zeta^{-}(x, y)+\omega\right) D_{\eta} \psi(x, y) e^{-\lambda(x \cdot e-c t)} \\
& +\left(2 \omega+\zeta^{-}(x, y)\right) D_{\eta} \psi(x, y) e^{-\lambda(x \cdot e-c t)} \\
> & 0
\end{aligned}
$$

from (3.12). The maximum principle yields $u(t, x, y) \leq \bar{u}_{\eta}(t, x, y)$ for all $(t, x, y) \in$ $[0,+\infty) \times \bar{\Omega}$ such that $x \cdot e-c t \geq \bar{\sigma}$. That completes the proof of Proposition 3.3

\subsection{A Liouville type result}

The last step before the proof of Theorem 1.3 is a Liouville type result for the time-global $(t \in \mathbb{R})$ solutions of $(1.1)$ which are trapped between two shifts of the front $\phi$ and which satisfy similar estimates to those in Proposition 3.3 . uniformly in time.

Proposition 3.4. In the notation of the previous subsections, let $v(t, x, y)$ be a solution of 1.1 , for all $(t, x, y) \in \mathbb{R} \times \bar{\Omega}$, such that

$$
\forall(t, x, y) \in \mathbb{R} \times \bar{\Omega}, \quad \phi(x \cdot e-c t+a, x, y) \leq v(t, x, y) \leq \phi(x \cdot e-c t+b, x, y),
$$

for some $b \leq 0 \leq a$. Assume also that for each $\eta>0$, there are $D_{\eta}>0$ and $\sigma_{\eta} \in \mathbb{R}$ such that, for all $(t, x, y) \in \mathbb{R} \times \bar{\Omega}$ with $x \cdot e-c t \geq \sigma_{\eta}$,

$$
\begin{aligned}
\phi(x \cdot e-c t & +\eta, x, y)-D_{\eta} \psi(x, y) e^{-\lambda(x \cdot e-c t)} \\
& \leq v(t, x, y) \leq \phi(x \cdot e-c t-\eta, x, y)+D_{\eta} \psi(x, y) e^{-\lambda(x \cdot e-c t)} .
\end{aligned}
$$

Then

$$
v(t, x, y)=\phi(x \cdot e-c t, x, y)=U(t, x, y) \quad \text { for all }(t, x, y) \in \mathbb{R} \times \bar{\Omega} .
$$

Proof. Define

$\eta^{*}=\min \left\{\eta \in[0,+\infty): v(t, x, y) \leq \phi\left(x \cdot e-c t-\eta^{\prime}, x, y\right)\right.$ in $\mathbb{R} \times \bar{\Omega}$ for all $\left.\eta^{\prime} \geq \eta\right\}$.

The real number $\eta^{*}$ is well-defined and it satisfies $0 \leq \eta^{*} \leq-b$, since $\phi_{s}<0$ in $\mathbb{R} \times \bar{\Omega}$. 
Let us now prove that $\eta^{*}=0$, which will imply that $u \leq U$ in $\mathbb{R} \times \bar{\Omega}$. Assume that $\eta^{*}>0$.

We first claim that there exists $\sigma^{*} \in\left[\sigma_{\eta^{*} / 4},+\infty\right)$ such that

$\forall(t, x, y) \in \mathbb{R} \times \bar{\Omega}, \quad\left[x \cdot e-c t \geq \sigma^{*}\right] \Rightarrow\left[v(t, x, y) \leq \phi\left(x \cdot e-c t-\eta^{*} / 2, x, y\right)\right]$,

where the real number $\sigma_{\eta^{*} / 4}$ is given by our assumption applied to $\eta=\eta^{*} / 4>0$. If not, then there exists a sequence $\left(t_{n}, x_{n}, y_{n}\right)_{n \in \mathbb{N}}$ in $\mathbb{R} \times \bar{\Omega}$ such that $s_{n}=x_{n} \cdot e-c t_{n} \rightarrow+\infty$ as $n \rightarrow+\infty$, and, for all $n \in \mathbb{N}$,

$$
\phi\left(s_{n}-\eta^{*} / 2, x_{n}, y_{n}\right)<\phi\left(s_{n}+\eta^{*} / 4, x_{n}, y_{n}\right)+D_{\eta^{*} / 4} \psi\left(x_{n}, y_{n}\right) e^{-\lambda s_{n}},
$$

from property 3.35 applied with $\eta=\eta^{*} / 4>0$. Thus,

$$
1<\frac{\phi\left(s_{n}-\eta^{*} / 4, x_{n}, y_{n}\right)-p^{-}\left(x_{n}, y_{n}\right)}{\phi\left(s_{n}-\eta^{*} / 2, x_{n}, y_{n}\right)-p^{-}\left(x_{n}, y_{n}\right)}+\frac{D_{\eta^{*} / 4} \psi\left(x_{n}, y_{n}\right) e^{-\lambda s_{n}}}{\phi\left(s_{n}-\eta^{*} / 2, x_{n}, y_{n}\right)-p^{-}\left(x_{n}, y_{n}\right)}
$$

for all $n \in \mathbb{N}$. The limsup as $n \rightarrow+\infty$ of the first term of the right-hand side is not larger than $e^{-\lambda_{m, \phi} \eta^{*} / 4}<1$ from $(2.2)$. The limit of the second term of the right-hand side is equal to 0 because of $(1.18)$ and $\lambda>\lambda_{c}$. A contradiction is reached as $n \rightarrow+\infty$. Therefore, property 3.36 holds for some $\sigma^{*} \geq \sigma_{\eta^{*} / 4}$.

Choose now $\sigma_{*} \leq \sigma^{*}$ such that

$$
\phi(s, x, y)>p^{+}(x, y)-\rho \quad \text { for all }(s, x, y) \in\left(-\infty, \sigma_{*}\right] \times \bar{\Omega},
$$

where $\rho>0$ is given in 1.5 .

We then claim that

$$
\inf _{(t, x, y) \in \mathbb{R} \times \bar{\Omega}, \sigma_{*} \leq x \cdot e-c t \leq \sigma^{*}}\left(\phi\left(x \cdot e-c t-\eta^{*}, x, y\right)-v(t, x, y)\right)>0 .
$$

Notice first that $v(t, x, y) \leq \phi\left(x \cdot e-c t-\eta^{*}, x, y\right)$ in $\mathbb{R} \times \bar{\Omega}$ by definition of $\eta^{*}$. Assume that the claim (3.38) is not true. Then there exists a sequence $\left(t_{n}, x_{n}, y_{n}\right)_{n \in \mathbb{N}}$ such that $s_{n}=x_{n} \cdot e-c t_{n} \in\left[\sigma_{*}, \sigma^{*}\right]$ for all $n \in \mathbb{N}$, and

$$
\phi\left(x_{n} \cdot e-c t_{n}-\eta^{*}, x_{n}, y_{n}\right)-v\left(t_{n}, x_{n}, y_{n}\right) \rightarrow 0 \quad \text { as } n \rightarrow+\infty .
$$

For each $n \in \mathbb{N}$, write $x_{n}=x_{n}^{\prime}+x_{n}^{\prime \prime}$, where $x_{n}^{\prime} \in L_{1} \mathbb{Z} \times \cdots \times L_{d} \mathbb{Z}$ and $\left(x_{n}^{\prime \prime}, y_{n}\right) \in C$, and

$$
v_{n}(t, x, y)=v\left(t+t_{n}, x+x_{n}^{\prime}, y\right) .
$$

Up to taking a subsequence, one can assume that, as $n \rightarrow+\infty, s_{n} \rightarrow s_{\infty} \in\left[\sigma_{*}, \sigma^{*}\right]$, $\left(x_{n}^{\prime \prime}, y_{n}\right) \rightarrow\left(x_{\infty}, y_{\infty}\right) \in C$ and $v_{n}(t, x, y) \rightarrow v_{\infty}(t, x, y)$ locally uniformly in $(t, x, y)$, where $v_{\infty}$ solves 1.1$]$ in $\mathbb{R} \times \bar{\Omega}$. We have $v_{n}(t, x, y) \leq \phi\left(x \cdot e-c t+x_{n}^{\prime} \cdot e-c t_{n}-\eta^{*}, x, y\right)$ since $\phi$ is periodic in $(x, y)$, whence

$$
v_{\infty}(t, x, y) \leq \phi\left(x \cdot e-c t+s_{\infty}-x_{\infty} \cdot e-\eta^{*}, x, y\right)
$$


for all $(t, x, y) \in \mathbb{R} \times \bar{\Omega}$. Furthermore, $v_{\infty}\left(0, x_{\infty}, y_{\infty}\right)=\phi\left(s_{\infty}-\eta^{*}, x_{\infty}, y_{\infty}\right)$ from 3.39. Hence,

$$
v_{\infty}(t, x, y)=\phi\left(x \cdot e-c t+s_{\infty}-x_{\infty} \cdot e-\eta^{*}, x, y\right) \quad \text { for all }(t, x, y) \in \mathbb{R} \times \bar{\Omega},
$$

from the strong maximum principle and periodicity of $\phi$ in the variables $(x, y)$. On the other hand, if $x \cdot e-c t \geq \sigma^{*}+c t_{n}-x_{n}^{\prime} \cdot e$, then

$$
v_{n}(t, x, y) \leq \phi\left(\left(x+x_{n}^{\prime}\right) \cdot e-c\left(t+t_{n}\right)-\eta^{*} / 2, x, y\right)
$$

from 3.36, whence

$$
\begin{aligned}
{\left[x \cdot e-c t \geq \sigma^{*}-s_{\infty}+x_{\infty} \cdot e\right] } & \\
& \Rightarrow\left[v_{\infty}(t, x, y) \leq \phi\left(x \cdot e-c t+s_{\infty}-x_{\infty} \cdot e-\eta^{*} / 2, x, y\right)\right] .
\end{aligned}
$$

This contradicts 3.40, since $\phi_{s}<0$ and $\eta^{*}>0$.

Therefore, property (3.38) holds. By continuity and $(x, y)$-periodicity of $\phi$, there exists $\eta_{*}$ such that $\eta^{*} / 2 \leq \eta_{*}<\eta^{*}$ and, for all $\eta \in\left[\eta_{*}, \eta^{*}\right]$,

$$
\left[\sigma_{*} \leq x \cdot e-c t \leq \sigma^{*}\right] \Rightarrow[v(t, x, y) \leq \phi(x \cdot e-c t-\eta, x, y)] .
$$

Actually, the previous inequality also holds when $x \cdot e-c t \geq \sigma^{*}$, because of 3.36 and $\phi_{s}<0$. Pick any $\eta$ in $\left[\eta_{*}, \eta^{*}\right]\left(\subset\left[0, \eta^{*}\right]\right)$. In the region where $x \cdot e-c t \leq \sigma_{*}$, we have $\phi(x \cdot e-c t-\eta, x, y)>p^{+}(x, y)-\rho$, from (3.37) and $\phi_{s}<0$. All assumptions of Lemma 2.1 are satisfied with $h=\sigma_{*}, \bar{U}(t, x, y)=\phi(x \cdot e-c t-\eta, x, y), \bar{\Phi}=$ $\phi(\cdot--\eta, \cdot, \cdot), \underline{U}=v$ and $\Phi(s, x, y)=v((x \cdot e-s) / c, x, y)$, apart from the fact that $\Phi$ may not be periodic in $(x, y)$. However, since $\Phi \leq \phi(\cdot+b, \cdot, \cdot)<p^{+}$, the arguments used in the proof of Lemma 2.1 (that is, Lemma 2.3 of [20]) can be immediately extended to the present case. They yield the inequality

$$
v(t, x, y) \leq \phi(x \cdot e-c t-\eta, x, y) \quad \text { for all }(t, x, y) \text { such that } x \cdot e-c t \leq \sigma_{*} .
$$

Eventually, $v(t, x, y) \leq \phi(x \cdot e-c t-\eta, x, y)$ in $\mathbb{R} \times \bar{\Omega}$ for all $\eta \in\left[\eta_{*}, \eta^{*}\right]$. Since $\eta_{*}<\eta^{*}$, that contradicts the minimality of $\eta^{*}$. Consequently, $\eta^{*}$ cannot be positive, which proves that $v(t, x, y) \leq \phi(x \cdot e-c t, x, y)$ in $\mathbb{R} \times \bar{\Omega}$.

The proof of the opposite inequality is exactly similar. Finally, $v(t, x, y)=$ $\phi(x \cdot e-c t, x, y)$ in $\mathbb{R} \times \bar{\Omega}$, which is the desired result.

Remark 3.5. Notice that the two key tools in the proof of Proposition 3.4 are first the property 2.2 , which holds for any pulsating front in the sense of (1.7), and second the fact that $e^{-\lambda s}=o\left(\phi(s, x, y)-p^{-}(x, y)\right)$ as $s \rightarrow+\infty$, uniformly in $(x, y) \in \bar{\Omega}$.

\subsection{Proof of Theorem 1.3}

With the results of the previous subsections, we are now able to complete the proof of Theorem 1.3 
Proof of Theorem 1.3. Assume that the limit (1.21) does not hold. Then there exist $\varepsilon>0$ and a sequence $\left(t_{n}, x_{n}, y_{n}\right)_{n \in \mathbb{N}}$ in $[0,+\infty) \times \bar{\Omega}$ such that $\lim _{n \rightarrow+\infty} t_{n}=+\infty$ and $\left|u\left(t_{n}, x_{n}, y_{n}\right)-U\left(t_{n}, x_{n}, y_{n}\right)\right| \geq \varepsilon$ for all $n \in \mathbb{N}$, that is,

$$
\left|u\left(t_{n}, x_{n}, y_{n}\right)-\phi\left(s_{n}, x_{n}, y_{n}\right)\right| \geq \varepsilon
$$

where $s_{n}=x_{n} \cdot e-c t_{n}$. Under the notations of Proposition 3.2, and using the monotonicity of $\phi$ in $s$, we obtain

$\phi\left(s_{n}+\sigma_{0}, x_{n}, y_{n}\right)-2 \varepsilon_{1}\|g\|_{\infty} e^{-\omega t_{n}} \leq u\left(t_{n}, x_{n}, y_{n}\right) \leq \phi\left(s_{n}-\sigma_{0}, x_{n}, y_{n}\right)+\|g\|_{\infty} e^{-\omega t_{n}}$.

If $s_{n} \rightarrow-\infty$, up to taking a subsequence, then

$$
\phi\left(s_{n}+\sigma_{0}, x_{n}, y_{n}\right)-p^{+}\left(x_{n}, y_{n}\right)-2 \varepsilon_{1}\|g\|_{\infty} e^{-\omega t_{n}} \leq u\left(t_{n}, x_{n}, y_{n}\right)-p^{+}\left(x_{n}, y_{n}\right) \leq 0,
$$

whence

$$
\lim _{n \rightarrow+\infty}\left(u\left(t_{n}, x_{n}, y_{n}\right)-p^{+}\left(x_{n}, y_{n}\right)\right)=0=\lim _{n \rightarrow+\infty}\left(\phi\left(s_{n}, x_{n}, y_{n}\right)-p^{+}\left(x_{n}, y_{n}\right)\right) .
$$

This contradicts (3.41). Therefore, the sequence $\left(s_{n}\right)_{n \in \mathbb{N}}$ is bounded from below. Similarly, one can prove that it is bounded from above.

For each $n \in \mathbb{N}$, write $x_{n}=x_{n}^{\prime}+x_{n}^{\prime \prime}$, where $x_{n}^{\prime} \in L_{1} \mathbb{Z} \times \cdots \times L_{d} \mathbb{Z}$ and $\left(x_{n}^{\prime \prime}, y_{n}\right) \in C$. Up to taking a subsequence, one can assume that $s_{n} \rightarrow s_{\infty} \in \mathbb{R},\left(x_{n}^{\prime \prime}, y_{n}\right) \rightarrow\left(x_{\infty}, y_{\infty}\right)$ $\in C$ as $n \rightarrow+\infty$. Set $t_{n}^{\prime}=t_{n}+\left(s_{\infty}-x_{\infty} \cdot e\right) / c$ and observe that $x_{n}^{\prime} \cdot e-c t_{n}^{\prime} \rightarrow 0$ as $n \rightarrow+\infty$. Denote

$$
u_{n}(t, x, y)=u\left(t+t_{n}^{\prime}, x+x_{n}^{\prime}, y\right) .
$$

Up to taking another subsequence, the functions $u_{n}$ converge locally uniformly in $\mathbb{R} \times \bar{\Omega}$ to a time-global solution $u_{\infty}$ of 1.1 in $\mathbb{R} \times \bar{\Omega}$. Furthermore, Proposition 3.2 implies that, for each $n \in \mathbb{N}$ and $(t, x, y) \in\left[-t_{n}^{\prime},+\infty\right) \times \bar{\Omega}$,

$$
\begin{aligned}
\phi(x \cdot e-c t & \left.+x_{n}^{\prime} \cdot e-c t_{n}^{\prime}+\sigma_{0}-\sigma_{0} e^{-\omega\left(t+t_{n}^{\prime}\right)}, x, y\right)-2 \varepsilon_{1}\|g\|_{\infty} e^{-\omega\left(t+t_{n}^{\prime}\right)} \leq u_{n}(t, x, y) \\
& \leq \phi\left(x \cdot e-c t+x_{n}^{\prime} \cdot e-c t_{n}^{\prime}-\sigma_{0}+\sigma_{0} e^{-\omega\left(t+t_{n}^{\prime}\right)}, x, y\right)+\|g\|_{\infty} e^{-\omega\left(t+t_{n}^{\prime}\right)},
\end{aligned}
$$

whence

$$
\phi\left(x \cdot e-c t+\sigma_{0}, x, y\right) \leq u_{\infty}(t, x, y) \leq \phi\left(x \cdot e-c t-\sigma_{0}, x, y\right)
$$

for all $(t, x, y) \in \mathbb{R} \times \bar{\Omega}$.

Let $\bar{\sigma} \in \mathbb{R}$ be as in Proposition 3.3 It follows that for each $\eta>0$, there is $D_{\eta}>0$ such that, for each $n \in \mathbb{N}$ and $(t, x, y) \in\left[-t_{n}^{\prime},+\infty\right) \times \bar{\Omega}$,

$$
\phi\left(x \cdot e-c t+x_{n}^{\prime} \cdot e-c t_{n}^{\prime}+\eta, x, y\right)-D_{\eta} \psi(x, y) e^{-\lambda\left(x \cdot e-c t+x_{n}^{\prime} \cdot e-c t_{n}^{\prime}\right)} \leq u_{n}(t, x, y)
$$

and

$$
\begin{aligned}
& {\left[\left(x+x_{n}^{\prime}\right) \cdot e-c\left(t+t_{n}^{\prime}\right) \geq \bar{\sigma}\right] \Rightarrow} \\
& \quad\left[u_{n}(t, x, y) \leq \phi\left(x \cdot e-c t+x_{n}^{\prime} \cdot e-c t_{n}^{\prime}-\eta, x, y\right)+D_{\eta} \psi(x, y) e^{-\lambda\left(x \cdot e-c t+x_{n}^{\prime} \cdot e-c t_{n}^{\prime}\right)}\right] .
\end{aligned}
$$


The passage to the limit as $n \rightarrow+\infty$ yields, for all $(t, x, y) \in \mathbb{R} \times \bar{\Omega}$ and $\eta>0$,

$$
\left\{\begin{array}{l}
\phi(x \cdot e-c t+\eta, x, y)-D_{\eta} \psi(x, y) e^{-\lambda(x \cdot e-c t)} \leq u_{\infty}(t, x, y) \\
{[x \cdot e-c t \geq \bar{\sigma}] \Rightarrow\left[u_{\infty}(t, x, y) \leq \phi(x \cdot e-c t-\eta, x, y)+D_{\eta} \psi(x, y) e^{-\lambda(x \cdot e-c t)}\right] .}
\end{array}\right.
$$

It finally follows from (3.42) and (3.43) and from Proposition 3.4 that $u_{\infty}(t, x, y)=$ $\phi(x \cdot e-c t, x, y)$ in $\mathbb{R} \times \Omega$ (we here apply a particular case of Proposition 3.4 when the real numbers $\sigma_{\eta}$ can all be set to $\bar{\sigma}$, independently of $\eta>0$ ). But assumption (3.41) implies that $\left|u_{n}\left(t_{n}-t_{n}^{\prime}, x_{n}^{\prime \prime}, y_{n}\right)-\phi\left(s_{n}, x_{n}^{\prime \prime}, y_{n}\right)\right| \geq \varepsilon$, whence

$$
\left|u_{\infty}\left(\left(-s_{\infty}+x_{\infty} \cdot e\right) / c, x_{\infty}, y_{\infty}\right)-\phi\left(s_{\infty}, x_{\infty}, y_{\infty}\right)\right| \geq \varepsilon .
$$

We have reached a contradiction. Hence, formula $(1.21)$ is proved and the proof of Theorem 1.3 is complete.

\section{Stability of KPP fronts with speeds $c^{*}(e)$}

This section is devoted to the proof of Theorem 1.5, under the KPP condition (1.6). Actually, because of 1.22 when $c>c^{*}(e)$, part (1) is an immediate consequence of Theorem 1.3. Only part (2) on the stability of KPP fronts with minimal speeds $c^{*}(e)$ remains to be proved. The proof follows the main scheme as that of Theorem 1.3. However, the ideas and the stability result are new even in the special cases which were previously considered in the literature. Two additional serious difficulties arise: firstly the sub- and super-solutions must take into account the fact that the behavior of the KPP fronts with minimal speeds $c^{*}(e)$ as they approach $p^{-}$is not purely exponential $e^{-\lambda^{*} s}$, secondly, because of the criticality of $\lambda^{*}$, some of the ideas used in Section 3 cannot just be adapted (for instance, there is no $\lambda$ satisfying (3.1) with $\lambda_{c}=\lambda^{*}$ ). The sub- and super-solutions involve products of exponentially decaying functions and suitable polynomial factors which are given in terms of some derivatives of the principal eigenfunctions $\psi_{\lambda}$ with respect to $\lambda$ at $\lambda=\lambda^{*}$.

Proof of part (2) of Theorem 1.5 Up to a shift in time, we can assume that $B=B_{\phi}$ in assumption 1.26, that is, $u_{0}(x, y)-p^{-}(x, y) \sim U(0, x, y)-p^{-}(x, y)$ as $x \cdot e \rightarrow+\infty$, where $B_{\phi}>0$ is given in formula 1.23 .

Step 1: Choice of parameters. Since $U$ is a pulsating front in the sense of (1.7) with speed $c^{*}(e)$, it follows from [20], as already underlined, that there exists a unique $\lambda^{*}>0$ such that $k\left(\lambda^{*}\right)+c^{*}(e) \lambda^{*}=0$ and $\lambda^{*}$ is a root of $k(\lambda)+\lambda c=0$ with multiplicity $2 m+2$. Furthermore, the function $\lambda \mapsto k(\lambda)$ is analytic (see [12, 28]) and, by the normalization condition (1.14) and standard elliptic estimates, the principal eigenfunctions $\psi_{\lambda}$ of the operators $L_{\lambda}$ given in 1.12 are also analytic with respect to $\lambda$ in the spaces $C^{2, \alpha}(\bar{\Omega})$. For each $j \in \mathbb{N}$ and $\lambda \in \mathbb{R}$, denote by $\psi_{\lambda}^{(j)}$ the $j$-th order derivative of $\psi_{\lambda}$ with respect to $\lambda$, under the convention that $\psi_{\lambda}^{(0)}=\psi_{\lambda}$. All these functions are periodic and of class $C^{2}$ 
in $\bar{\Omega}$. Denote by $L_{\lambda}^{(j)}$ the operator whose coefficients are the $j$-th order derivatives with respect to $\lambda$ of the coefficients of $L_{\lambda}$. In other words,

$L_{\lambda}^{(0)} \psi=L_{\lambda} \psi, L_{\lambda}^{(1)} \psi=2 e A \nabla \psi+[\nabla \cdot(A e)-q \cdot e-2 \lambda e A e] \psi, L_{\lambda}^{(2)} \psi=-2 e A e \psi$

and $L_{\lambda}^{(j)} \psi=0$ for all $j \geq 3$ and for all $\psi \in C^{2}(\bar{\Omega})$ and $\lambda \in \mathbb{R}$. Differentiating the relation $L_{\lambda} \psi_{\lambda}-k(\lambda) \psi_{\lambda}=0$ with respect to $\lambda$ yields

$$
\left\{\begin{array}{l}
L_{\lambda} \psi_{\lambda}^{(1)}-k(\lambda) \psi_{\lambda}^{(1)}+2 e A \nabla \psi_{\lambda}+[\nabla \cdot(A e)-q \cdot e-2 \lambda e A e] \psi_{\lambda}-k^{\prime}(\lambda) \psi_{\lambda} \\
=\left(L_{\lambda}-k(\lambda)\right) \psi_{\lambda}^{(1)}+\left(L_{\lambda}^{(1)}-k^{\prime}(\lambda)\right) \psi_{\lambda}=0 \\
L_{\lambda} \psi_{\lambda}^{(j)}-k(\lambda) \psi_{\lambda}^{(j)}+j\left(2 e A \nabla \psi_{\lambda}^{(j-1)}+[\nabla \cdot(A e)-q \cdot e-2 \lambda e A e] \psi_{\lambda}^{(j-1)}\right) \\
-j k^{\prime}(\lambda) \psi_{\lambda}^{(j-1)}-2 C_{j}^{2} e A e \psi_{\lambda}^{(j-2)}-\sum_{2 \leq l \leq j} C_{j}^{l} k^{(l)}(\lambda) \psi_{\lambda}^{(j-l)} \\
=\left(L_{\lambda}-k(\lambda)\right) \psi_{\lambda}^{(j)}+j\left(L_{\lambda}^{(1)}-k^{\prime}(\lambda)\right) \psi_{\lambda}^{(j-1)}+C_{j}^{2} L_{\lambda}^{(2)} \psi_{\lambda}^{(j-2)}-\sum_{2 \leq l \leq j} C_{j}^{l} k^{(l)}(\lambda) \psi_{\lambda}^{(j-l)} \\
=0 \quad \text { for all } j \geq 2,
\end{array}\right.
$$

where $\mathrm{C}_{n}^{m}=n ! /(m !(n-m) !)$ for all integers $m, n$ such that $m \leq n$. Similarly, since $\nu A \nabla \psi_{\lambda}=\lambda(\nu A e) \psi_{\lambda}$ on $\partial \Omega$ for all $\lambda \in \mathbb{R}$, one finds that, for all $\lambda \in \mathbb{R}$,

$$
v A \nabla \psi_{\lambda}^{(j)}-\lambda(v A e) \psi_{\lambda}^{(j)}-j(v A e) \psi_{\lambda}^{(j-1)}=0 \quad \text { on } \partial \Omega \text { for all } j \geq 1 .
$$

Let $i$ and $I$ be the functions defined by

$$
\begin{aligned}
& i(s, x, y)=B_{\phi} e^{-\lambda^{*} s}\left[\sum_{j=0}^{2 m+1}(-1)^{j} C_{2 m+1}^{j} s^{2 m+1-j} \psi_{\lambda^{*}}^{(j)}(x, y)\right], \\
& I(t, x, y)=i\left(x \cdot e-c^{*}(e) t, x, y\right) .
\end{aligned}
$$

Notice that

$$
i(s, x, y) \sim B_{\phi} e^{-\lambda^{*} s} s^{2 m+1} \psi_{\lambda^{*}}(x, y) \sim \phi(s, x, y)-p^{-}(x, y) \quad \text { as } s \rightarrow+\infty,
$$

uniformly in $(x, y) \in \bar{\Omega}$, from $\sqrt{1.23}$ and the fact that $\min _{\bar{\Omega}} \psi_{\lambda^{*}}>0$. It also follows from (4.1) and (4.2) applied to $\lambda=\lambda^{*}$ that $\nu A(x, y) \nabla I(t, x, y)=0$ for all $(t, x, y) \in$ $\mathbb{R} \times \partial \Omega$, and

$$
I_{t}-\nabla \cdot(A(x, y) \nabla I)+q(x, y) \cdot \nabla I-\zeta^{-}(x, y) I=0 \quad \text { in } \mathbb{R} \times \bar{\Omega} .
$$

Now, for $\mu \in \mathbb{R}$ and $a>0$, denote

$$
\begin{aligned}
& h(s, x, y)=e^{-\mu s}\left[\sum_{j=0}^{2 m+2}(-1)^{j} C_{2 m+2}^{j}(s+a)^{2 m+2-j} \psi_{\mu}^{(j)}(x, y)\right], \\
& H(t, x, y)=h\left(x \cdot e-c^{*}(e) t, x, y\right) .
\end{aligned}
$$


By of 4.2), $v A(x, y) \nabla H(t, x, y)=0$ for $(t, x, y) \in \mathbb{R} \times \partial \Omega$. Notice that

$$
h(s, x, y) \sim e^{-\mu s} s^{2 m+2} \psi_{\mu}(x, y) \quad \text { as } s \rightarrow+\infty, \text { uniformly in }(x, y) \in \bar{\Omega} .
$$

It also follows from the definition of $m$ and from the proof of Proposition 4.5 of [20] that one can choose $\mu-\lambda^{*}>0$ small enough and $a>0$ large enough so that

$$
\left\{\begin{array}{l}
h_{s}(s, x, y) \leq 0 \quad \text { for all }(s, x, y) \in[0,+\infty) \times \bar{\Omega} \\
H_{t}-\nabla \cdot(A(x, y) \nabla H)+q(x, y) \cdot \nabla H-\zeta^{-}(x, y) H \leq-v e^{-\mu s}(s+a)^{2 m+2}<0
\end{array}\right.
$$

for all $(t, x, y) \in \mathbb{R} \times \bar{\Omega}$ such that $s=x \cdot e-c^{*}(e) t \geq 0$, where

$$
v=\frac{\left|k^{(2 m+2)}\left(\lambda^{*}\right)\right| \kappa^{*}\left(\mu-\lambda^{*}\right)^{2 m+2}}{4(2 m+2) !}>0 \quad \text { and } \quad \kappa^{*}=\min _{\bar{\Omega}} \psi_{\lambda^{*}}>0
$$

Decreasing $\mu-\lambda^{*}$ if necessary, one can also assume without loss of generality that

$$
\lambda^{*}<\mu<\lambda^{*}(1+\beta),
$$

where one recalls that $\beta>0$ is such that the function $(x, y, u) \mapsto \frac{\partial f}{\partial u}\left(x, y, p^{-}(x, y)+u\right)$ is of class $C^{0, \beta}(\bar{\Omega} \times[0, \gamma])$ for some $\gamma>0$.

Let $\theta$ be a $C^{2}(\bar{\Omega})$ nonpositive periodic function satisfying $(3.4)$. Let $\psi^{+}$be given by 1.17 , and denote $m^{+}=\min _{\bar{\Omega}} \psi^{+}>0$. Because of 1.23 and $\mu>\lambda^{*}$, one can choose $\underline{s} \geq 1$ such that

$$
0 \leq h(s, x, y) \leq \frac{\phi(s, x, y)-p^{-}(x, y)}{2} \leq m^{+} \quad \text { for all }(s, x, y) \in[\underline{s}-1,+\infty) \times \bar{\Omega} .
$$

Let $\chi \in C^{2}(\mathbb{R} ;[0,1])$ be as in 3.6 and let $g$ be the function defined in $\mathbb{R} \times \bar{\Omega}$ by

$$
g(s, x, y)=-h(s, x, y) \chi(s+\theta(x, y))+\psi^{+}(x, y)(1-\chi(s+\theta(x, y))) .
$$

Notice that $\chi(s+\theta(x, y))=0$ for all $(s, x, y) \in(-\infty, \underline{s}-1] \times \bar{\Omega}$, and that $g$ is bounded, $C^{2}$ in $\mathbb{R} \times \bar{\Omega}$ and periodic with respect to the variables $(x, y)$. Furthermore, $g \geq-m^{+}$in $\mathbb{R} \times \bar{\Omega}$, and, for all $(s, x, y) \in \mathbb{R} \times \bar{\Omega}$,

$$
\left\{\begin{array}{l}
s \leq \underline{s}-1 \Rightarrow g(s, x, y)=\psi^{+}(x, y) \geq 0 \\
s \geq \underline{s}-1 \Rightarrow g(s, x, y) \geq-h(s, x, y) \geq-\frac{\phi(s, x, y)-p^{-}(x, y)}{2}
\end{array}\right.
$$

We then claim that

$$
\limsup _{\varsigma \rightarrow-\infty} \sup _{(s, x, y) \in \mathbb{R} \times \bar{\Omega}, \rho \in\left(0, \rho^{+} / 2\right]} \frac{\phi(s, x, y)-\rho g(s+\varsigma, x, y)-p^{+}(x, y)}{\rho \psi^{+}(x, y)} \leq-1,
$$


where $\rho^{+}=\min _{\bar{\Omega}}\left[\left(p^{+}-p^{-}\right) / \psi^{+}\right]>0$. Assume not. Then there exist $0<\varepsilon \leq 1$ and sequences $\left(s_{n}, x_{n}, y_{n}\right)_{n \in \mathbb{N}}$ in $\mathbb{R} \times \bar{\Omega},\left(\rho_{n}^{\prime}\right)_{n \in \mathbb{N}}$ in $\left(0, \rho^{+} / 2\right]$ and $\left(\varsigma_{n}\right)_{n \in \mathbb{N}}$ such that $\lim _{n \rightarrow+\infty} \varsigma_{n}=-\infty$ and

$$
\frac{\phi\left(s_{n}, x_{n}, y_{n}\right)-\rho_{n}^{\prime} g\left(s_{n}+\varsigma_{n}, x_{n}, y_{n}\right)-p^{+}\left(x_{n}, y_{n}\right)}{\rho_{n}^{\prime} \psi^{+}\left(x_{n}, y_{n}\right)} \geq-1+\varepsilon
$$

for all $n \in \mathbb{N}$. As in the proof of Lemma 3.1, it follows that the sequence $\left(s_{n}+\varsigma_{n}\right)_{n \in \mathbb{N}}$ is bounded from below, whence $\lim _{n \rightarrow+\infty} s_{n}=+\infty$. The last part of the argument is different from that of Lemma 3.1. since $g$ is not nonnegative anymore. For each $n \in \mathbb{N}$,

$$
\begin{aligned}
\frac{\rho^{+}}{2}+\frac{\phi\left(s_{n}, x_{n}, y_{n}\right)-p^{+}\left(x_{n}, y_{n}\right)}{\psi^{+}\left(x_{n}, y_{n}\right)} & \geq \frac{\rho_{n}^{\prime} m^{+}}{\psi^{+}\left(x_{n}, y_{n}\right)}+\frac{\phi\left(s_{n}, x_{n}, y_{n}\right)-p^{+}\left(x_{n}, y_{n}\right)}{\psi^{+}\left(x_{n}, y_{n}\right)} \\
& \geq-(1-\varepsilon) \rho_{n}^{\prime} \geq-(1-\varepsilon) \frac{\rho^{+}}{2}>-\frac{\rho^{+}}{2}
\end{aligned}
$$

since $0<\rho_{n}^{\prime} \leq \rho^{+} / 2$, and $g \geq-m^{+}$in $\mathbb{R} \times \bar{\Omega}$. This leads to a contradiction as in Lemma 3.1. Therefore, one can choose $s_{0} \leq 0$ such that

$$
\frac{\phi(s, x, y)-\rho g\left(s+s_{0}, x, y\right)-p^{+}(x, y)}{\psi^{+}(x, y)} \leq-\frac{\rho}{2}
$$

for all $\rho \in\left(0, \rho^{+} / 2\right]$ and $(s, x, y) \in \mathbb{R} \times \bar{\Omega}$.

Set

$$
G(t, x, y)=g\left(x \cdot e-c^{*}(e) t, x, y\right) e^{-\omega t}
$$

for all $(t, x, y) \in \mathbb{R} \times \bar{\Omega}$, where

$$
\omega=\mu^{+} / 2>0
$$

and $\mu^{+}>0$ is as in 1.17 . The function $G$ is of class $C^{2}(\mathbb{R} \times \bar{\Omega})$ and, by $\sqrt{4.6}$, there exists a continuous, bounded and $(x, y)$-periodic function $B$ in $\mathbb{R} \times \bar{\Omega}$ such that

$$
G_{t}-\nabla \cdot(A(x, y) \nabla G)+q(x, y) \cdot \nabla G=B\left(x \cdot e-c^{*}(e) t, x, y\right) e^{-\omega t},
$$

where

$$
\begin{cases}B(s, x, y) \geq\left(-\zeta^{-}(x, y)+\omega\right) h(s, x, y) & \text { if } s \geq \underline{s}+\|\theta\|_{\infty}(\geq 0) \\ B(s, x, y)=\left(\zeta^{+}(x, y)+\mu^{+}-\omega\right) \psi^{+}(x, y) & \text { if } s \leq \underline{s}-1\end{cases}
$$

For all $(s, x, y) \in \mathbb{R} \times \bar{\Omega}$, define

$$
C(s, x, y)=h_{s}(s, x, y) \chi(s+\theta(x, y))-\left(h(s, x, y)+\psi^{+}(x, y)\right) \chi^{\prime}(s+\theta(x, y)) .
$$

The function $C$ is continuous and bounded in $\mathbb{R} \times \bar{\Omega}$, and periodic in the variables $(x, y)$. Observe that $C(s, x, y) \sim-\mu e^{-\mu s} s^{2 m+2} \psi_{\mu}(x, y)$ as $s \rightarrow+\infty$, uniformly in $(x, y) \in \bar{\Omega}$, 
whence $C(s, x, y)=o\left(-\phi_{s}(s, x, y)\right)$ as $s \rightarrow+\infty$ from 1.23, (2.2) and $\mu>\lambda^{*}$. Choose $\rho^{-}>0$ as in 3.12 and $s^{+} \geq 0$ such that

$$
\forall(s, x, y) \in\left[s^{+},+\infty\right) \times \bar{\Omega}, \quad\left\{\begin{array}{l}
p^{-}(x, y)<\phi(s, x, y) \leq p^{-}(x, y)+\rho^{-} / 2, \\
-\rho^{-} / 2 \leq g\left(s+s_{0}, x, y\right)=-h\left(s+s_{0}, x, y\right) \leq 0, \\
B\left(s+s_{0}, x, y\right) \geq\left(-\zeta^{-}(x, y)+\omega\right) h\left(s+s_{0}, x, y\right), \\
C\left(s+s_{0}, x, y\right)=h_{s}\left(s+s_{0}, x, y\right) \leq 0, \\
|C(s, x, y)| \leq-\phi_{s}(s, x, y) .
\end{array}\right.
$$

Lastly, define

$$
M=\max _{(x, y) \in \bar{\Omega}, p^{-}(x, y)-\|g\|_{\infty} \leq u \leq p^{+}(x, y)+\|g\|_{\infty}}\left|\frac{\partial f}{\partial u}(x, y, u)\right| \geq 0,
$$

let $\rho_{1}^{+}>0, s^{-} \leq 0$ and $\delta>0$ be as in 3.14 - 3.16 , and set

$\varepsilon_{1}=\min \left(\frac{\rho_{1}^{+}}{4}, \frac{\delta}{4\|C\|_{\infty}}, \frac{1}{2}\right)>0, \quad \varepsilon_{0}=m^{+} \varepsilon_{1}>0, \quad \underline{\sigma}=\frac{M\|g\|_{\infty}+\|B\|_{\infty}}{\omega\|C\|_{\infty}} \geq 0$.

Step 2: Comparison with sub-and super-solutions. Assume now that the initial condition $u_{0}$ satisfies 1.19 and 1.20 . For all $(t, x, y) \in[0,+\infty) \times \bar{\Omega}$, define

$$
\begin{aligned}
& \underline{u}(t, x, y)=\phi\left(s_{\sigma_{0}}(t, x), x, y\right)-2 \varepsilon_{1} g\left(s_{\sigma_{0}}(t, x)+s_{0}, x, y\right) e^{-\omega t}, \\
& \bar{u}(t, x, y)=\phi\left(s_{-\sigma_{0}}(t, x), x, y\right)+2 \varepsilon_{1} g\left(s_{-\sigma_{0}}(t, x), x, y\right) e^{-\omega t},
\end{aligned}
$$

where $s_{\kappa}(t, x)=x \cdot e-c^{*}(e) t+\kappa-\kappa e^{-\omega t}$ and $\sigma_{0}$ will be chosen below.

As in Step 1 of the proof of Proposition 3.2. one can choose $t_{0}>0$ such that 3.25 holds. Then we claim that

$$
\max \left\{\underline{u}\left(t_{0}, x, y\right), p^{-}(x, y)\right\} \leq u\left(t_{0}, x, y\right) \quad \text { in } \bar{\Omega},
$$

for $\sigma_{0}$ large enough. If not, there exist sequences $\left(x_{n}, y_{n}\right)_{n \in \mathbb{N}}$ in $\bar{\Omega}$ and $\left(\sigma_{n}\right)_{n \in \mathbb{N}}$ such that $\lim _{n \rightarrow+\infty} \sigma_{n}=+\infty$ and

$$
\phi\left(s_{\sigma_{n}}\left(t_{0}, x_{n}\right), x_{n}, y_{n}\right)-2 \varepsilon_{1} g\left(s_{\sigma_{n}}\left(t_{0}, x_{n}\right)+s_{0}, x_{n}, y_{n}\right) e^{-\omega t_{0}}>u\left(t_{0}, x_{n}, y_{n}\right)
$$

for all $n \in \mathbb{N}$. As in Step 2 of the proof of Proposition 3.2, it follows that $\lim _{n \rightarrow+\infty} s_{\sigma_{n}}\left(t_{0}, x_{n}\right)=+\infty$ and $\lim _{n \rightarrow+\infty} x_{n} \cdot e=+\infty$. We have

$$
\begin{array}{r}
\frac{\phi\left(s_{\sigma_{n}}\left(t_{0}, x_{n}\right), x_{n}, y_{n}\right)-p^{-}\left(x_{n}, y_{n}\right)}{U\left(t_{0}, x_{n}, y_{n}\right)-p^{-}\left(x_{n}, y_{n}\right)}-\frac{2 \varepsilon_{1} g\left(s_{\sigma_{n}}\left(t_{0}, x_{n}\right)+s_{0}, x_{n}, y_{n}\right) e^{-\omega t_{0}}}{U\left(t_{0}, x_{n}, y_{n}\right)-p^{-}\left(x_{n}, y_{n}\right)} \\
>\frac{u\left(t_{0}, x_{n}, y_{n}\right)-p^{-}\left(x_{n}, y_{n}\right)}{U\left(t_{0}, x_{n}, y_{n}\right)-p^{-}\left(x_{n}, y_{n}\right)} .
\end{array}
$$


The right-hand side converges to 1 as $n \rightarrow+\infty$, from 3.23 , while the first term of the left-hand side converges to 0 , from 2.2 . Lastly, the second term on the left-hand converges to 0 too, from 1.23 and $\mu>\lambda^{*}$. This leads to a contradiction, which yields 4.14) for $\sigma_{0}$ large enough.

Similarly,

$$
u\left(t_{0}, x, y\right) \leq \min \left\{\bar{u}\left(t_{0}, x, y\right), p^{+}(x, y)\right\} \quad \text { in } \bar{\Omega}
$$

for $\sigma_{0}$ large enough. Assume not. Then there exist sequences $\left(x_{n}, y_{n}\right)_{n \in \mathbb{N}}$ in $\bar{\Omega}$ and $\left(\sigma_{n}\right)_{n \in \mathbb{N}}$ such that $\lim _{n \rightarrow+\infty} \sigma_{n}=+\infty$ and

$$
\phi\left(s_{-\sigma_{n}}\left(t_{0}, x_{n}\right), x_{n}, y_{n}\right)+2 \varepsilon_{1} g\left(s_{-\sigma_{n}}\left(t_{0}, x_{n}\right), x_{n}, y_{n}\right) e^{-\omega t_{0}}<u\left(t_{0}, x_{n}, y_{n}\right)
$$

for all $n \in \mathbb{N}$. As in Step 2 of the proof of Proposition 3.2, it follows that the sequence $\left(s_{-\sigma_{n}}\left(t_{0}, x_{n}\right)\right)_{n \in \mathbb{N}}$ is bounded from below, whence $\lim _{n \rightarrow+\infty} x_{n} \cdot e=+\infty$ and $\lim _{n \rightarrow+\infty}\left(u\left(t_{0}, x_{n}, y_{n}\right)-p^{-}\left(x_{n}, y_{n}\right)\right)=0$. Since $\phi>p^{-}$and $\varepsilon_{1} \in(0,1 / 2]$, it follows from 4.9 that $2 \varepsilon_{1} g\left(s_{-\sigma_{n}}\left(t_{0}, x_{n}\right), x_{n}, y_{n}\right) e^{-\omega t_{0}} \geq-\left(\phi\left(s_{-\sigma_{n}}\left(t_{0}, x_{n}\right), x_{n}, y_{n}\right)-\right.$ $\left.p^{-}\left(x_{n}, y_{n}\right)\right) / 2$, whence

$$
\frac{\phi\left(s_{-\sigma_{n}}\left(t_{0}, x_{n}\right), x_{n}, y_{n}\right)-p^{-}\left(x_{n}, y_{n}\right)}{2\left(U\left(t_{0}, x_{n}, y_{n}\right)-p^{-}\left(x_{n}, y_{n}\right)\right)}<\frac{u\left(t_{0}, x_{n}, y_{n}\right)-p^{-}\left(x_{n}, y_{n}\right)}{U\left(t_{0}, x_{n}, y_{n}\right)-p^{-}\left(x_{n}, y_{n}\right)} .
$$

One gets a contradiction as in Step 2 of the proof of Proposition 3.2

As a consequence, 4.15 holds for $\sigma$ large enough and one can choose $\sigma_{0} \geq \underline{\sigma}$ large enough so that 4.14) and (4.15) hold for $\sigma=\sigma_{0}$.

Let us now check that $\underline{u}$ and $\bar{u}$ are respectively sub- and super-solutions of 1.1 , for $t \geq t_{0}$, when $\underline{u}>p^{-}$and $\bar{u}<p^{+}$. Notice first that $v A(x, y) \nabla \underline{u}(t, x, y)=$ $\nu A(x, y) \nabla \bar{u}(t, x, y)=0$ for $(x, y) \in \partial \Omega$, from (3.4), 4.2) and the definition of $g$. It follows from 4.10 and the definition of $s_{\sigma_{0}}(t, x)$ that

$$
\begin{aligned}
\mathcal{L} \underline{u}(t, x, y)= & f\left(x, y, \phi\left(s_{\sigma_{0}}(t, x), x, y\right)\right)-f(x, y, \underline{u}(t, x, y)) \\
& +\sigma_{0} \omega \phi_{s}\left(s_{\sigma_{0}}(t, x), x, y\right) e^{-\omega t}-2 \varepsilon_{1} B\left(s_{\sigma_{0}}(t, x)+s_{0}, x, y\right) e^{-\omega t} \\
& -2 \varepsilon_{1} \sigma_{0} \omega C\left(s_{\sigma_{0}}(t, x)+s_{0}, x, y\right) e^{-2 \omega t} .
\end{aligned}
$$

If $\underline{u}(t, x, y)>p^{-}(x, y)$ and $s_{\sigma_{0}}(t, x) \geq s^{+}$, where $s^{+}$is given by 4.11 , then it follows from the first four properties of (4.11) and from the inequalities $0<\varepsilon_{1} \leq 1 / 2$ and $\phi_{s}<0$ that $\mathcal{L} \underline{u}(t, x, y) \leq 0$. If $\underline{u}(t, x, y)>p^{-}(x, y)$ and $s_{\sigma_{0}}(t, x) \leq s^{-}$, where $s^{-}$is given by 3.15, then it follows from 3.15, $\phi_{s}<0, s_{0} \leq 0$ and $\omega=\mu^{+} / 2$ that $\mathcal{L} \underline{u}(t, x, y) \leq 0$. Lastly, if $p^{-}(x, y)<\underline{u}(t, x, y)$ and $s^{-} \leq s_{\sigma_{0}}(t, x) \leq s^{+}$, then it follows from 3.16, (4.12), 4.13) and $\sigma_{0} \geq \underline{\sigma}$ that $\mathcal{L} \underline{u}(t, x, y) \leq 0$ too. Since $\underline{u}\left(t_{0}, x, y\right) \leq u\left(t_{0}, x, y\right)$ and $p^{-}(x, y) \leq u(t, x, y)$ for all $(t, x, y) \in[0,+\infty) \times \bar{\Omega}$, one concludes from the parabolic maximum principle that

$$
\max \left\{\underline{u}(t, x, y), p^{-}(x, y)\right\} \leq u(t, x, y) \quad \text { for all }(t, x, y) \in\left[t_{0},+\infty\right) \times \bar{\Omega} \text {. }
$$


Similarly,

$$
\begin{aligned}
\mathcal{L} \bar{u}(t, x, y)= & f\left(x, y, \phi\left(s_{-\sigma_{0}}(t, x), x, y\right)\right)-f(x, y, \bar{u}(t, x, y)) \\
& +\sigma_{0} \omega \phi_{s}\left(s_{-\sigma_{0}}(t, x), x, y\right) e^{-\omega t}+2 \varepsilon_{1} B\left(s_{-\sigma_{0}}(t, x), x, y\right) e^{-\omega t} \\
& -2 \varepsilon_{1} \sigma_{0} \omega C\left(s_{-\sigma_{0}}(t, x), x, y\right) e^{-2 \omega t} .
\end{aligned}
$$

As above, it then follows from (3.15), 3.16, 4.11, 4.12), (4.13), and from $\phi_{s}<0$, $s_{0} \leq 0, \omega=\mu^{+} / 2$ and $\sigma_{0} \geq \underline{\sigma}$ that $\mathcal{L} \bar{u}(t, x, y) \geq 0$ for all $(t, x, y) \in\left[t_{0},+\infty\right) \times \bar{\Omega}$ such that $\bar{u}(t, x, y)<p^{+}(x, y)$. Since $\bar{u}\left(t_{0}, x, y\right) \geq u\left(t_{0}, x, y\right)$ and $u(t, x, y) \leq p^{+}(x, y)$ for all $(t, x, y) \in[0,+\infty) \times \bar{\Omega}$, one concludes from the parabolic maximum principle that

$$
u(t, x, y) \leq \min \left\{\bar{u}(t, x, y), p^{+}(x, y)\right\} \quad \text { for all }(t, x, y) \in\left[t_{0},+\infty\right) \times \bar{\Omega} .
$$

Step 3: Time-global sharp estimates as $x \cdot e-c^{*}(e) t$ is very large. We now claim that, for any $\eta>0$, there are $D_{\eta}>0$ and $\sigma_{\eta} \in \mathbb{R}$ such that

$$
\begin{aligned}
& \forall(t, x, y) \in[0,+\infty) \times \bar{\Omega}, \quad\left[x \cdot e-c^{*}(e) t \geq \sigma_{\eta}\right] \Rightarrow \\
& \quad\left[\phi\left(x \cdot e-c^{*}(e) t+\eta, x, y\right)-D_{\eta} \psi_{\lambda^{*}}(x, y) e^{-\lambda^{*}\left(x \cdot e-c^{*}(e) t\right)}\right. \\
& \left.\quad \leq u(t, x, y) \leq \phi\left(x \cdot e-c^{*}(e) t-\eta, x, y\right)+D_{\eta} \psi_{\lambda^{*}}(x, y) e^{-\lambda^{*}\left(x \cdot e-c^{*}(e) t\right)}\right] .
\end{aligned}
$$

Let $\eta>0$. We are going to trap $u$, for very large $x \cdot e-c^{*}(e) t$, between a sub- and a super-solution which are larger and smaller than the left- and right-hand sides of 4.16, respectively.

Define, for all $(t, x, y) \in[0,+\infty) \times \bar{\Omega}$,

$$
\underline{u}_{\eta}(t, x, y)=i(s+\eta / 2, x, y)+h(s, x, y)-D_{\eta} \psi_{\lambda^{*}}(x, y) e^{-\lambda^{*} s}+p^{-}(x, y),
$$

where $s=x \cdot e-c^{*}(e) t, i$ and $h$ have been defined in Step 1, and the real number $D_{\eta}>0$ will be chosen later. Recall now that the function $(x, y, \xi) \mapsto \frac{\partial f}{\partial u}\left(x, y, p^{-}(x, y)+\xi\right)$ is assumed to be of class $C^{0, \beta}(\bar{\Omega} \times[0, \gamma])$ for some $\beta, \gamma>0$. Therefore, there exists $r \geq 0$ such that

$$
\left|f\left(x, y, p^{-}(x, y)+\xi\right)-f\left(x, y, p^{-}(x, y)\right)-\zeta^{-}(x, y) \xi\right| \leq r \xi^{1+\beta}
$$

for all $(x, y, \xi) \in \bar{\Omega} \times[0, \gamma]$. From 1.24 with $B=B_{\phi}, 44.3$, , 4.5) and 4.8 , there exists $\sigma_{\eta} \geq 0$ such that

$$
\left\{\begin{array}{l}
0<\phi(s+\eta, x, y)-p^{-}(x, y) \leq i(s+\eta / 2, x, y)+h(s, x, y) \leq \gamma \\
0<h(s, x, y) \leq i(s+\eta / 2, x, y) \\
r 2^{1+\beta} i(s+\eta / 2, x, y)^{1+\beta} \leq v e^{-\mu s}(s+a)^{2 m+2}
\end{array}\right.
$$

for all $(s, x, y) \in\left[\sigma_{\eta},+\infty\right) \times \bar{\Omega}$, where $v>0$ is as in 4.7 , and

$$
i(x \cdot e+\eta / 2, x, y)+h(x \cdot e, x, y) \leq u_{0}(x, y)-p^{-}(x, y)
$$


for all $(x, y) \in \bar{\Omega}$ such that $x \cdot e \geq \sigma_{\eta}$. Then choose $D_{\eta}>0$ large enough so that

$$
i\left(\sigma_{\eta}+\eta / 2, x, y\right)+h\left(\sigma_{\eta}, x, y\right)-D_{\eta} \psi_{\lambda^{*}}(x, y) e^{-\lambda^{*} \sigma_{\eta}} \leq 0
$$

for all $(x, y) \in \bar{\Omega}$. In order to prove the first inequality of 4.16 , it is then enough to prove, from (4.18), that

$$
\underline{u}_{\eta}(t, x, y) \leq u(t, x, y) \quad \text { for all }(t, x, y) \in[0,+\infty) \times \bar{\Omega} \text { with } x \cdot e-c^{*}(e) t \geq \sigma_{\eta} .
$$

Observe that

$$
\underline{u}_{\eta}(0, x, y) \leq u_{0}(x, y) \quad \text { for all }(x, y) \in \bar{\Omega} \text { such that } x \cdot e \geq \sigma_{\eta},
$$

from 4.19 , and that

$$
\begin{aligned}
\underline{u}_{\eta}(t, x, y) \leq p^{-}(x, y) & \leq u(t, x, y) \\
& \text { for all }(t, x, y) \in[0,+\infty) \times \bar{\Omega} \text { with } x \cdot e-c^{*}(e) t=\sigma_{\eta},
\end{aligned}
$$

from 4.20). Moreover, $v A(x, y) \nabla \underline{u}_{\eta}(t, x, y)=0$ for all $(t, x, y) \in[0,+\infty) \times \partial \Omega$, since $\nu A(x, y) \nabla I=v A(x, y) \nabla H=v A(x, y) \nabla p^{-}=\nu A(x, y) \nabla \psi_{\lambda^{*}}-\lambda^{*}(\nu A(x, y) e) \psi_{\lambda^{*}}$ $=0$ for all $(x, y) \in \partial \Omega$. Lastly, recall that $u \geq p^{-}$. Therefore, from the parabolic maximum principle, in order to prove 44.21 , it is enough to check that $\underline{L}_{\eta}(t, x, y) \leq 0$ for all $(t, x, y) \in \Omega_{\eta}^{-}$, where

$$
\Omega_{\eta}^{-}=\left\{(t, x, y) \in[0,+\infty) \times \bar{\Omega}: x \cdot e-c^{*}(e) t \geq \sigma_{\eta} \text { and } \underline{u}_{\eta}(t, x, y)>p^{-}(x, y)\right\} .
$$

From 4.4, (4.6) and $L_{\lambda^{*}} \psi_{\lambda^{*}}=k\left(\lambda^{*}\right) \psi_{\lambda^{*}}$ in $\bar{\Omega}$, it is straightforward to see that

$$
\begin{aligned}
\mathcal{L}_{u_{\eta}}(t, x, y) \leq & \zeta^{-}(x, y)\left(i(s+\eta / 2, x, y)+h(s, x, y)-D_{\eta} \psi \lambda^{*}(x, y) e^{-\lambda^{*} s}\right) \\
& -v e^{-\mu s}(s+a)^{2 m+2}+f\left(x, y, p^{-}(x, y)\right)-f\left(x, y, \underline{u}_{\eta}(t, x, y)\right)
\end{aligned}
$$

for all $(t, x, y) \in \Omega_{\eta}^{-}$, where $s=x \cdot e-c^{*}(e) t$. From $4.18,0<\underline{u}_{\eta}(t, x, y)-p^{-}(x, y)$ $\leq \gamma$ for all $(t, x, y) \in \Omega_{\eta}^{-}$, whence

$$
\begin{aligned}
f\left(x, y, \underline{u}_{\eta}(t, x, y)\right) \geq & f\left(x, y, p^{-}(x, y)\right)+\zeta^{-}(x, y)\left(\underline{u}_{\eta}(t, x, y)-p^{-}(x, y)\right) \\
& -r\left(\underline{u}_{\eta}(t, x, y)-p^{-}(x, y)\right)^{1+\beta} .
\end{aligned}
$$

Furthermore, $0<\underline{u}_{\eta}(t, x, y)-p^{-}(x, y) \leq 2 i\left(x \cdot e-c^{*}(e) t+\eta / 2, x, y\right)$ in $\Omega_{\eta}^{-}$. It follows that, for all $(t, x, y) \in \Omega_{\eta}^{-}$,

$$
\mathcal{L} \underline{u}_{\eta}(t, x, y) \leq-v e^{-\mu s}(s+a)^{2 m+2}+r 2^{1+\beta} i(s+\eta / 2, x, y)^{1+\beta} \leq 0
$$

from 4.18). As a consequence, 4.21 holds, and hence the first inequality in 4.16 as well. 
Define now, for all $(t, x, y) \in[0,+\infty) \times \bar{\Omega}$,

$$
\bar{u}_{\eta}(t, x, y)=i(s-\eta / 2, x, y)+D_{\eta} \psi_{\lambda^{*}}(x, y) e^{-\lambda^{*} s}+p^{-}(x, y),
$$

where $s=x \cdot e-c^{*}(e) t$. After increasing $\sigma_{\eta}$ and $D_{\eta}$ if necessary, it follows from 1.24 with $B=B_{\phi}$, 4.3) and (4.8) that one can assume that 4.18)-4.20) hold, as well as

$$
\left\{\begin{array}{l}
\forall(s, x, y) \in\left[\sigma_{\eta},+\infty\right) \times \bar{\Omega}, \quad 0<i(s-\eta / 2, x, y) \leq \phi(s-\eta, x, y)-p^{-}(x, y), \\
\forall(x, y) \in \bar{\Omega}, \quad\left[x \cdot e \geq \sigma_{\eta}\right] \Rightarrow\left[u_{0}(x, y)-p^{-}(x, y) \leq i(x \cdot e-\eta / 2, x, y)\right], \\
\forall(x, y) \in \bar{\Omega}, \quad i\left(\sigma_{\eta}-\eta / 2, x, y\right)+D_{\eta} \psi_{\lambda^{*}}(x, y) e^{-\lambda^{*} \sigma_{\eta}}+p^{-}(x, y) \geq p^{+}(x, y) .
\end{array}\right.
$$

In order to prove the second inequality of (4.16), it is thus enough to prove that

$$
u(t, x, y) \leq \bar{u}_{\eta}(t, x, y) \quad \text { for all }(t, x, y) \in[0,+\infty) \times \bar{\Omega} \text { with } x \cdot e-c^{*}(e) t \geq \sigma_{\eta} .
$$

It also follows from 4.22 that

$$
u_{0}(x, y) \leq \bar{u}_{\eta}(0, x, y) \quad \text { for all }(x, y) \in \bar{\Omega} \text { such that } x \cdot e \geq \sigma_{\eta},
$$

and

$u(t, x, y) \leq p^{+}(x, y) \leq \bar{u}_{\eta}(t, x, y)$ for all $(t, x, y) \in[0,+\infty) \times \bar{\Omega}$ with $x \cdot e-c^{*}(e) t=\sigma_{\eta}$.

Moreover, $v A(x, y) \nabla \bar{u}_{\eta}(t, x, y)=0$ for all $(t, x, y) \in[0,+\infty) \times \partial \Omega$. Recall that $u \leq p^{+}$. Therefore, from the parabolic maximum principle, in order to prove 4.23, it is enough to check that $\mathcal{L} \bar{u}_{\eta}(t, x, y) \geq 0$ for all $(t, x, y) \in \Omega_{\eta}^{+}$, where

$$
\Omega_{\eta}^{+}=\left\{(t, x, y) \in[0,+\infty) \times \bar{\Omega}: x \cdot e-c^{*}(e) t \geq \sigma_{\eta} \text { and } \bar{u}_{\eta}(t, x, y)<p^{+}(x, y)\right\} .
$$

From 4.4, from $L_{\lambda^{*}} \psi_{\lambda^{*}}=k\left(\lambda^{*}\right) \psi_{\lambda^{*}}$ and from the KPP condition 1.6,

$$
\begin{aligned}
\mathcal{L} \bar{u}_{\eta}(t, x, y)= & \zeta^{-}(x, y)\left(i(s-\eta / 2, x, y)+D_{\eta} \psi_{\lambda^{*}}(x, y) e^{-\lambda^{*} s}\right) \\
& +f\left(x, y, p^{-}(x, y)\right)-f\left(x, y, \underline{u}_{\eta}(t, x, y)\right) \\
\geq & 0
\end{aligned}
$$

for all $(t, x, y) \in \Omega_{\eta}^{+}$, where $s=x \cdot e-c^{*}(e) t$. As a consequence, 4.23 holds, and hence the second inequality in 4.16 as well.

Step 4: Conclusion. By using the fact that $e^{-\lambda^{*} s}=o\left(\phi(s, x, y)-p^{-}(x, y)\right)$ as $s \rightarrow+\infty$ uniformly in $(x, y) \in \bar{\Omega}$, it follows from the same arguments as in Proposition 3.4 that, if $v(t, x, y)$ is a solution of 1.1 in $\mathbb{R} \times \bar{\Omega}$ such that

$$
\left\{\begin{array}{l}
\exists a \geq b \in \mathbb{R}, \\
\phi\left(x \cdot e-c^{*}(e) t+a, x, y\right) \leq v(t, x, y) \leq \phi\left(x \cdot e-c^{*}(e) t+b, x, y\right) \text { in } \mathbb{R} \times \bar{\Omega}, \\
\forall \eta>0, \exists D_{\eta}>0, \exists \sigma_{\eta} \in \mathbb{R}, \quad\left[s=x \cdot e-c^{*}(e) t \geq \sigma_{\eta}\right] \Rightarrow \\
{\left[\phi(s+\eta, x, y)-D_{\eta} \psi_{\lambda^{*}}(x, y) e^{-\lambda^{*} s} \leq v(t, x, y) \leq \phi(s-\eta, x, y)+D_{\eta} \psi_{-\lambda^{*}}(x, y) e^{-\lambda^{*} s}\right],}
\end{array}\right.
$$

then $v(t, x, y)=\phi(s, x, y)=U(t, x, y)$ in $\mathbb{R} \times \bar{\Omega}$. 
Finally, from this Liouville type result and Steps 2 and 3, property 1.25 of part (2) of Theorem 1.5 (with $\tau=0$ due to our assumption $B=B_{\phi}$ ) can be deduced by the same arguments as those used in the proof of property (1.21) of Theorem 1.3 The proof of Theorem 1.5 is thus complete.

Acknowledgments. The first author is indebted to the Alexander von Humboldt Foundation for its support. Both authors are also supported by the French "Agence Nationale de la Recherche" within projects ColonSGS, PREFERED, and URTICLIM (second author).

\section{References}

[1] Bages, M., Martinez, P., Roquejoffre, J.-M.: Dynamique en grand temps pour une classe d'équations de type KPP en milieu périodique. C. R. Math. Acad. Sci. Paris I 346, 10511056 (2008) Zbl 1156.35322 MR 2462047

[2] Berestycki, H., Hamel, F.: Front propagation in periodic excitable media. Comm. Pure Appl. Math. 55, 949-1032 (2002) Zbl 1024.37054 MR 1900178

[3] Berestycki, H., Hamel, F.: Reaction-Diffusion Equations and Propagation Phenomena. Appl. Math. Sci., Springer, to appear

[4] Berestycki, H., Hamel, F., Nadirashvili, N.: The principal eigenvalue of elliptic operators with large drift and applications to nonlinear propagation phenomena. Comm. Math. Phys. 253, 451-480 (2005) Zbl 1123.35033 MR 2140256

[5] Berestycki, H., Hamel, F., Nadirashvili, N.: The speed of propagation for KPP type problems. I: Periodic framework. J. Eur. Math. Soc. 7, 173-213 (2005) Zbl 1142.35464 MR 2127993

[6] Berestycki, H., Hamel, F., Roques, L.: Analysis of the periodically fragmented environment model: I: Species persistence. J. Math. Biol. 51, 75-113 (2005) Zbl 1066.92047 MR 2214420

[7] Berestycki, H., Hamel, F., Roques, L.: Analysis of the periodically fragmented environment model: II: Biological invasions and pulsating traveling fronts. J. Math. Pures Appl. 84, 11011146 (2005) Zbl 1083.92036 MR 2155900

[8] Berestycki, H., Nirenberg, L.: On the method of moving planes and the sliding method. Bol. Soc. Brasil. Mat. 22, 1-37 (1991) Zbl 0784.35025 MR 1159383

[9] Berestycki, H., Nirenberg, L.: Travelling fronts in cylinders. Ann. Inst. H. Poincaré Anal. Non Linéaire 9, 497-572 (1992) Zbl 0799.35073 MR 1191008

[10] Bramson, M.: Convergence of solutions of the Kolmogorov equation to travelling waves. Mem. Amer. Math. Soc. 44, no. 285 (1983) Zbl 0517.60083 MR 0705746

[11] Cabré, X., Roquejoffre, J.-M.: Propagation de fronts dans les équations de Fisher-KPP avec diffusion fractionnaire. C. R. Math. Acad. Sci. Paris 347, 1361-1366 (2009) Zbl 1182.35072 MR 2588782

[12] Conca, C., Vanninathan, M.: Homogenization of periodic structures via Bloch decomposition. SIAM J. Appl. Math. 57, 1639-1659 (1997) Zbl 0990.35019 MR 1484944

[13] El Smaily, M.: Pulsating traveling fronts: Asymptotics and homogenization regimes. Eur. J. Appl. Math. 19, 393-434 (2008) Zbl 1162.35064 MR 2431698

[14] El Smaily, M., Hamel, F., Roques, L.: Homogenization and influence of fragmentation in a biological invasion model. Discrete Contin. Dynam. Systems 25, 321-342 (2009) Zbl 1179.35047 MR 2525180

[15] Fife, P. C., McLeod, J. B.: The approach of solutions of non-linear diffusion equations to traveling front solutions. Arch. Ration. Mech. Anal. 65, 335-361 (1977) Zbl 0361.35035 MR 0442480 
[16] Fisher, R. A.: The advance of advantageous genes. Ann. Eugenics 7, 355-369 (1937) JFM 63.1111 .04

[17] Fréjacques, G.: Travelling waves in infinite cylinders with time-periodic coefficients. Ph.D. Thesis (2005)

[18] Gallay, T.: Local stability of critical fronts in nonlinear parabolic partial differential equations. Nonlinearity 7, 741-764 (1994) Zbl 0801.35046 MR 1275528

[19] Guo, J.-S., Wu, C.-C.: Uniqueness and stability of traveling waves for periodic monostable lattice dynamical system. J. Differential Equations 246, 3818-3833 (2009) Zbl pre05555282 MR 2514727

[20] Hamel, F.: Qualitative properties of monostable pulsating fronts: exponential decay and monotonicity. J. Math. Pures Appl. 89, 355-399 (2008) Zbl 1171.35061 MR 2401143

[21] Hamel, F., Nadin, G.: Spreading properties and complex dynamics for monostable reactiondiffusion equations. Preprint

[22] Hamel, F., Roques, L.: Fast propagation for KPP equations with slowly decaying initial conditions. J. Differential Equations 249, 1726-1745 (2010) Zbl pre05793319 MR 2677813

[23] Hamel, F., Sire, Y.: Spreading speeds for some reaction-diffusion equations with general initial conditions. SIAM J. Math. Anal., to appear

[24] Heinze, S.: Large convection limits for KPP fronts. Preprint

[25] Hudson, W., Zinner, B.: Existence of traveling waves for reaction-diffusion equations of Fisher type in periodic media. In: Boundary Problems for Functional Differential Equations, World Sci., 187-199 (1995) Zbl 0846.35062 MR 1375475

[26] Kametaka, Y.: On the nonlinear diffusion equation of Kolmogorov-Petrovski-Piskunov type. Osaka J. Math. 13, 11-66 (1976) Zbl 0344.35050 MR 0422875

[27] Kanel', Ya. I.: Stabilization of solution of the Cauchy problem for equations encountered in combustion theory. Mat. Sb. 59, 245-288 (1962) (in Russian) Zbl 0152.10302 MR 0157130

[28] Kato, T.: Perturbation Theory for Linear Operators. 2nd ed., Springer (1984) Zbl 0531.47014 MR 0407617

[29] Kolmogorov, A. N., Petrovskiǔ, I. G., Piskunov, N. S.: Étude de l'équation de la diffusion avec croissance de la quantité de matière et son application à un problème biologique, Bull. Univ. État Moscou Sér. Intern. A 1, no. 6, 1-26 (1937) Zbl 0018.32106

[30] Larson, D. A.: Transient bounds and time-asymptotic behavior of solutions to nonlinear equations of Fisher type. SIAM J. Appl. Math. 34, 93-103 (1978) Zbl 0373.35036 MR 0482838

[31] Lau, K.-S.: On the nonlinear diffusion equation of Kolmogorov, Petrovskij, and Piscunov. J. Differential Equations 59, 44-70 (1985) Zbl 0584.35091| MR 0803086

[32] Levermore, C. D., Xin, J. X.: Multidimensional stability of traveling waves in a bistable reaction-diffusion equation, II. Comm. Partial Differential Equations 17, 1901-1924 (1999) Zbl 0789.35020 MR 1194744

[33] Mallordy, J.-F., Roquejoffre, J.-M.: A parabolic equation of the KPP type in higher dimensions. SIAM J. Math. Anal. 26, 1-20 (1995) Zbl 0813.35041 MR 1311879

[34] Matano, H.: Traveling waves in spatially inhomogeneous diffusive media-The non-periodic case. Preprint

[35] Murray, J. D.: Mathematical Biology, 3rd ed. in 2 vols.: I. An Introduction. Springer (2002) (corr. 2nd printing 2008); II. Spatial Models and Biomedical Applications. Springer (2003) Zbl 1006.92001 Zbl 1006.92002 MR 1908418 MR 1952568

[36] Nadin, G.: Travelling fronts in space-time periodic media. J. Math. Pures Appl. 92, 232-262 (2009) Zbl 1182.35074 MR 2555178

[37] Nolen, J., Xin, J.: Reaction-diffusion front speeds in spatially-temporally periodic shear flows. SIAM Multiscale Model. Simul. 1, 554-570 (2003) Z Zbl 1053.35068 $\mid$ MR 2029591 
[38] Nolen, J., Xin, J.: Existence of KPP type fronts in space-time periodic shear flows and a study of minimal speeds based on variational principle. Discrete Contin. Dynam. Systems 13, 1217-1234 (2005) Zbl 1097.35072 MR 2166666

[39] Novikov, A., Ryzhik, L.: Boundary layers and KPP fronts in a cellular flow. Arch. Ration. Mech. Anal. 184, 23-48 (2007) Zbl 1109.76064 MR 2289862

[40] Roquejoffre, J.-M.: Eventual monotonicity and convergence to traveling fronts for the solutions of parabolic equations in cylinders. Ann. Inst. H. Poincaré Anal. Non Linéaire 14, 499-552 (1997) Zbl 0884.35013 MR 1464532

[41] Ryzhik, L., Zlatoš, A.: KPP pulsating front speed-up by flows. Comm. Math. Sci. 5, 575-593 (2007) Zbl 1152.35055 MR 2352332

[42] Sattinger, D. H.: Stability of waves of nonlinear parabolic systems. Adv. Math. 22, 312-355 (1976) Zbl 0344.35051 MR 0435602

[43] Shigesada, N., Kawasaki, K.: Biological Invasions: Theory and Practice. Oxford Univ. Press (1997)

[44] Uchiyama, K.: The behavior of solutions of some nonlinear diffusion equations for large time. J. Math. Kyoto Univ. 18, 453-508 (1978) Zbl 0408.35053 MR 0509494

[45] van Saarloos, W.: Front propagation into unstable states. Phys. Rep. 386, 29-222 (2003) Zbl 1042.74029

[46] Weinberger, H. F.: On spreading speeds and traveling waves for growth and migration in periodic habitat. J. Math. Biol. 45, 511-548 (2002) Zbl $1058.92036 \mid$ MR 1943224

[47] Xin, X.: Existence and stability of traveling waves in periodic media governed by a bistable nonlinearity. J. Dynam. Differential Equations 3, 541-573 (1991) Zbl 0769.35033 MR 1129560

[48] Xin, X.: Existence of planar flame fronts in convective-diffusive periodic media. Arch. Ration. Mech. Anal. 121, 205-233 (1992) Zbl 0764.76074 MR 1188981

[49] Xin, J.: Front propagation in heterogeneous media. SIAM Rev. 42, 161-230 (2000) Zbl 0951.35060 MR 1778352

[50] Zlatoš, A.: Sharp asymptotics for KPP pulsating front speed-up and diffusion enhancement by flows. Arch. Ration. Mech. Anal. 195, 441-453 (2010) Zbl 1185.35205 MR 2592283 